\author{
UNIVERSIDADE DE SÃO PAULO \\ INSTITUTO DE FÍSICA DE SÃO CARLOS
}

JÉSSICA ARAB MARCOMINI

Estudo da possibilidade de detecção de matéria escura com telescópios Cherenkov

São Carlos

2015 



\section{Estudo da possibilidade de detecção de matéria escura com telescópios Cherenkov}

Dissertação apresentada ao Programa de Pós-Graduação em Física do Instituto de Física de São Carlos da Universidade de São Paulo, para obtenção do título de Mestre em Ciências.

Área de Concentração: Física Básica

Orientador: Prof. Dr. Luiz Vitor de Souza

Filho

\section{Versão Corrigida}

(Versão original disponível na Unidade que aloja o Programa)

São Carlos

2015 
AUTORIZO A REPRODUÇÃO E DIVULGAÇÃO TOTAL OU PARCIAL DESTE TRABALHO, POR QUALQÜER MEIO CONVENCIONAL OU ELETRÔNICO PARA FINS DE ESTUDO E PESQUISA, DESDE QUE CITADA A FONTE.

Ficha catalográfica elaborada pelo Serviço de Biblioteca e Informação do IFSC, com os dados fornecidos pelo(a) autor(a)

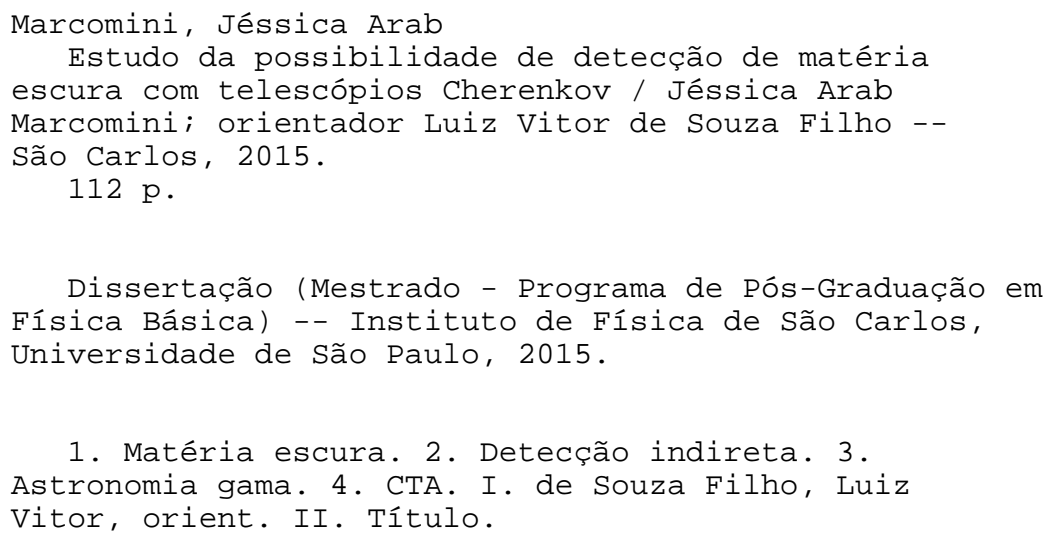




\section{Agradecimentos}

Gostaria de agradecer primeiramente minha família por todo apoio em todas as situações possíveis da minha vida, especialmente por ter lidado com minhas oscilações de humor durante o período de graduação (e outros). Sem vocês, eu não estaria aqui. À minha mãe e melhor amiga Ana Lúcia Arab Marcomini e ao meu irmão e modelo Leandro Arab Marcomini, não me imagino sem suas influências durante esses anos.

À todos amigos do Diocesano, cresci com vocês e tornei-me o que sou por causa de vocês. Não conseguiria pedir melhor ambiente e crescimento durante essa fase do que o que vocês proporcionaram. Devo minha sanidade e "pé no chão"a vocês: Aninha, Bruno, Ana, Paola, Gabi, Amanda e Sarto.

Quero agradecer a turma de Física 09 em geral e aos agregados FisComp/Fisica 10 a todos os momentos da graduação. Desde os desesperos compartilhados e listas infinitas até os palquinhos e conversas de "cincão". Não imaginei que encontraria pessoas como vocês durante a graduação, e sou eternamente grata por esses anos. À Nathalia, Millena, Everton (Kavera) e a todos os outros nomes que deveriam ser mencionados, agradeço pelo crescimento pessoal e por todas as risadas.

Ao meu melhor amigo e marido Iberê Oliveira Kuntz de Souza, quero dizer que todos os desesperos, listas, palquinhos e "cincão"não teriam sido os mesmos sem você. E que agradeço muito todas as formas de apoio e companheirismo que você foi e é hoje para mim. Não existe um "punho dos brothers"que combine mais com o meu.

Ao grupo de astrofísica, Jessica, Raul, Rodrigo, Rita e Carlos, agradeço a amizade e as infinitas soluções para os códigos do Root.

Ao meu orientador Prof. Dr. Luiz Vitor de Souza Filho, agradeço a paciência e compreensão durante esses dois anos e meio de mestrado e ao apoio para conclusão da dissertação.

Agradeço à USP pelo crescimento profissional durante todos esses anos e à CAPES pelo apoio financeiro durante o mestrado. 



\section{Resumo}

MARCOMINI, J. A. Estudo da possibilidade de detecção de matéria escura com telescópios Cherenkov. 2015. 112p. Dissertação (Mestrado em Ciências) - Instituto de Física de São Carlos, Universidade de São Paulo, São Carlos, 2015.

A existência de matéria escura é sustentada pela observação de efeitos gravitacionais sobre a matéria comum. A partir desses efeitos, com medidas de curvas de rotação e lentes gravitacionais, é possível calcular a densidade de matéria escura necessária para causá-los. Para descrever o comportamento observado, foram criados alguns modelos teóricos, porém a natureza das partículas que constituem matéria escura continua desconhecida. Determinar propriedades como massa e seção de choque da possível partícula de matéria escura é fundamental para o entendimento da natureza de seus efeitos sobre matéria bariônica. No âmbito experimental, os Telescópios Cherenkov medem a radiação gama proveniente do cosmo com energia entre $\mathrm{GeV}-\mathrm{TeV}$ de forma que uma possível interação (como exemplo a aniquilação de partículas de matéria escura) poderia ter seu resultado final de raios gama detectado em um dos experimentos de observação indireta. Neste trabalho de mestrado analisamos os conceitos de matéria escura a partir de um modelo específico de partículas WIMPs, o neutralino. Estudamos galáxias anãs esferoidais como possíveis fontes do sinal de raios gama proveniente da aniquilação de neutralinos. Para o entendimento dos cálculos, reproduzimos os resultados de dois experimentos importantes para a área, pertencentes aos telescópios VERITAS e MAGIC, validando as implementações realizadas. Estudamos também galáxias anãs esferoidais observadas pelo experimento FERMI-LAT para as quais limites superiores de fluxo foram determinados. Fizemos uso dessas medidas e extrapolamos os espectros de energias para o intervalo a ser observado pelo CTA. Utilizando curvas de sensitividade realistas para uma possível configuração do CTA, determinamos a potencialidade de detectação de matéria escura pelo CTA de 18 fontes estudados pelo FERMI-LAT. A partir dos resultados obtidos com a simulação do Observatório, podemos concluir quais fontes proporcionam melhores avanços para as pesquisas envolvendo matéria escura com o modelo escolhido.

Palavras-chave: Matéria escura. Detecção indireta. Astronomia gama. CTA. 



\section{Abstract}

MARCOMINI, J. A. Study of the possibility of dark matter detection with Cherenkov telescopes. 2015. 112p. Dissertação (Mestrado em Ciências) - Instituto de Física de São Carlos, Universidade de São Paulo, São Carlos, 2015.

The existence of dark matter is sustained by the observation of its gravitational effects on ordinary matter. By studying these effects, with rotation curves and gravitational lensing measurements, it is possible to calculate the dark matter density necessary to cause them. Theoretical models were created to describe the observed behavior, however the nature of the constituent particles is still unknown. Determining the particles' properties such as mass and cross section is fundamental for the understanding of the nature of its effects on baryonic matter. On the experimental scope, Cherenkov Telescopes measure the gamma radiation coming from the cosmo with an energy between $\mathrm{GeV}-\mathrm{TeV}$ making it possible for an interaction to have its final product detected in one of these experiments. In this dissertation, we present an analysis of dark matter concepts considering a specific model of WIMPs particles, represented by the neutralino. We studied dwarf spheroidal galaxies as possible gamma-ray flux sources originated from the neutralino annihilation. We reproduced the results on annihilation cross section of two important experiments for this particular field (VERITAS and MAGIC), validating the codes implemented. This was perfomed with the objective of understanding the calculus involved. We studied dwarf spheroidal galaxies observed by the FERMI-LAT experiment for which upper limits flux were determined. We used these measurements and extrapolated the energy spectrum to the one to be observed by CTA. With realistic sensitivity curves for a possible CTA configuration, we determined the potencial for a dark matter detection for 18 sources studied by FERMI-LAT. With the results obtained with the Observatory simulation, we were able to conclude which of these sources represent improvements for dark matter researches with the specific model described.

Keywords: Dark matter. Indirect detection. Gamma astronomy. CTA. 



\section{LISTA DE FIGURAS}

Figura 2.1 - Função $v=r^{-\frac{1}{2}}$ descritiva para um comportamento kepleriano, ou seja, tipicamente deveríamos ver uma versão parecida e suavizada para a velocidade da matéria que constitui uma galáxia.

Figura 2.2 - Curva de rotação de NGC 6503 representada pela linha sólida com os dados experimentais. A linha tracejada representa a velocidade do conteúdo luminoso, a pontilhada representa a velocidade do gás, e a curva tracejada-pontilhada a velocidade inferida para a contribuição de matéria escura no sistema.

Figura 2.3 - Aglomerado de galáxias Bullet. Na esquerda, a imagem óptica mostra os contornos de matéria gravitacional inferidos por lentes gravitacionais. À direita, a imagem em raio-x do aglomerado mostra claramente a diferença de densidade de matéria visível e escura.

Figura 2.4 - Esquema representativo sobre as possíveis conversões ALP-fóton.

Figura 2.5 - Curvas de limites superiores da seção de choque de aniquilação determinadas para diferentes fontes e experimentos.

Figura 2.6 - Representação dos métodos de detecção e processos de interação de partículas de matéria escura. A partícula entitulada $\chi$ representa a partícula de matéria escura e a entitulada $M P$, partículas do Modelo Padrão

Figura 3.1 - Limites do valor da seção de choque ao longo dos anos determinados por diferentes experimentos.

Figura 3.2 - Representação do cone de visualização de uma fonte extensa com abertura $\theta$. A esfera roxa representa a fonte com extensão de raio $r$ e distância até o observador $D$.

Figura 3.3 - Taxas de produção de raios gama para uma partícula de massa $500 \mathrm{GeV}$ aniquilando em $b \bar{b}$ (linha sólida) e em $\tau^{+} \tau^{-}$(linha tracejada).

Figura 4.1 - Comparação de sensitividade do Observatório CTA com a de outros observatórios existentes para um tempo de observação de $50 \mathrm{~h}$. 
Figura 4.2 - Exemplos de distribuição dos telescópios LST, MST e SST para os diferentes arranjos B, C e E. O tamanho das circunferências está em escala com o tamanho real dos espelhos de $24 \mathrm{~m}, 12 \mathrm{~m}, 10 \mathrm{~m}$ e $7 \mathrm{~m}$. . . 55

Figura 4.3 - Sensitividades dos arranjos B, C e E. Para cada arranjo, note que há um regime preferencial de atuação conforme aumentamos a energia. . . 56

Figura 4.4 - Relação de sensitividade dos tipos de telescópios LSTs, MSTs e SSTS com seus respectivos regimes de atuação para o arranjo $E(50 \mathrm{~h}, 5 \sigma$, ruído de fundo de $5 \%$ e mínimo de detecção de 10 eventos). A curva preta indica a sensitividade resultante da combinação dos três tipos. . . 56

Figura 4.5 - Sensitividade do CTA para os arranjos A-K para um sinal com significância $5 \sigma$, com um mínimo de 10 eventos detectados e com um ruído de fundo constituindo $5 \%$ do sinal. O gráfico de cima é simulado para um ângulo zenital de $20^{\circ}$ e o de baixo, para $50^{\circ}$.

Figura 4.6 - Sensitividade do arranjo E para três tempos de observação diferentes com um sinal de significância $5 \sigma$, possuindo um mínimo de 10 eventos detectados e uma contribuição de $5 \%$ de ruído de fundo.

Figura 4.7 - Curvas de sensitividade do CTA sobre seção de choque de aniquilação por massa da partícula de matéria escura com significância $5 \sigma$ e com tempo de observação $T=100 \mathrm{~h}$ para o arranjo $\mathrm{E}$.

Figura 4.8 - Curva de fluxo de raios gama do aglomerado de galáxias Fornax. A curva vermelha representa a contribuição de raios cósmicos para o sinal, a curva azul, a contribuição de matéria escura e em preto, a soma das duas.

Figura 4.9 - Sensitividade do CTA para a seção de choque de aniquilação da partícula de matéria escura no Centro Galáctico para os arranjos E e B. As curvas sólidas correspondem ao canal de aniquilação $b \bar{b}$, as pontilhadas ao canal $\mu^{+} \mu^{-}$e as tracejadas ao canal $\tau^{+} \tau^{-}$.

Figura 5.1 - Limites superiores do fluxo diferencial observados pelo MAGIC com diferentes expoentes da lei de potências estimada. A linha sólida representa o fluxo diferencial da Nebulosa de Carangueijo, a reta com longos traços representa $10 \%$ desse fluxo e a com traços menores $1 \%$. 
Figura 5.2 - Limites superiores do fluxo integral considerando os diferentes expoentes da lei de potências. As linhas tracejadas representam as funções aproximadas para os valores de limite superior de fluxo.

Figura 5.3 - Perfis da taxa de produção de raios gama $\frac{\mathrm{d} N_{\gamma}}{\mathrm{d} E}$ para massas de $100 \mathrm{GeV}$, $1000 \mathrm{GeV}$ e $10000 \mathrm{GeV}$ assumindo o canal de aniquilação $b \bar{b}$ (em cima) e $\tau^{+} \tau^{-}$(embaixo). As curvas tracejadas representam as funções resultantes da convolução sobre as energias do modelo gerado e medidas.

Figura 5.4 - Perfil de produção de raios gama gerado para o canal de aniquilação $b \bar{b}$ com massa $m_{\chi}=1000 \mathrm{GeV}$.

Figura 5.5 - Perfis de produção de raios gama $\left(\frac{\mathrm{d} N_{\gamma}}{\mathrm{d} E}\right)$ considerando massas de $316 \mathrm{GeV}$ (curva preta), $1 \mathrm{TeV}$ (curva vermelha), $3.16 \mathrm{TeV}$ (curva verde) e $10 \mathrm{TeV}$ (curva azul). Todas as curvas são provenientes da aniquilação de neutralinos pelas contribuições $90 \% b \bar{b}$ e $10 \% \tau^{+} \tau^{-}$.

Figura 5.6 - Perfil de produção de raios gama gerado para uma partícula de massa $m_{\chi}=1000 \mathrm{GeV}$ aniquilando com contribuições de $90 \%$ do canal $b \bar{b}$ e $10 \% \tau^{+} \tau^{-}$.

Figura 5.7 - Curvas de limite superior de seção de choque pela massa da partícula derivados para Draco (curva azul) e Segue 1 (curva vermelha). . . . . . 73

Figura 6.1 - Simulação de resposta do CTA de fluxo diferencial por energia de uma fonte emitindo $10 \%$ do fluxo correspondente a nebulosa de Carangueijo. À direita, podemos ver o mapeamento da emissão da fonte em questão. 78

Figura 6.2 - Curva de $x^{1.5} \frac{\mathrm{d} N}{\mathrm{~d} x}$ por $x$, onde $x=\frac{E}{m_{\chi}}$, para a massa de $2 T e V$ gerada com Mathematica.

Figura 6.3 - Curvas de fluxo diferencial mínimo detectável por energia geradas para as 8 massas diferentes, para a fonte Sculptor $\left(J=8.9 \cdot 10^{11} \mathrm{TeV}^{2} \mathrm{~cm}^{-5} \mathrm{sr}\right)$ com significância $5 \sigma$, mínimo de 10 eventos por intervalo de energia e com $0.05 \%$ de ruído de fundo. Da esquerda para a direita, curvas geradas com massa de $0.8 \mathrm{TeV}, 1 \mathrm{TeV}, 2 \mathrm{TeV}$ e $2.5 \mathrm{TeV}$, e abaixo com massas de $5 \mathrm{TeV}, 10 \mathrm{TeV}, 15 \mathrm{TeV}$ e $20 \mathrm{TeV}$

Figura 6.4 - Curva da seção de choque por massa para a galáxia Sculptor com diferentes tempos de observação. 
Figura 6.5 - Curva da seção de choque por massa para a galáxia Ursa Minor com diferentes tempos de observação.

Figura 6.6 - Curva da seção de choque por massa para a galáxia Segue I com diferentes tempos de observação.

Figura 6.7 - Sensitividade correspondente à configuração de $3 \sigma$, número mínimo de eventos correspondente a 7 e ruído de fundo equivalente a $0.03 \%$ do sinal representada pela curva em verde. As retas em preto foram argumentos de entrada do programa a fim de validar a região de detecção com as características descritas pela sensitividade da curva verde.

Figura 6.8 - Sensitividade correspondente à configuração de $5 \sigma$, número mínimo de eventos correspondente a 10 e ruído de fundo equivalente a $0.05 \%$ do sinal representada pela curva em vermelho. Também testamos a validade da curva de sensitividade com as funções correspondentes as retas em preto.

Figura 6.9 - Curvas de seção de choque de aniquilação por massa para as 18 fontes estudadas com duas configurações diferentes: significância $3 \sigma$, mínimo de eventos 7 e ruído de fundo $0.03 \%$ e significância $5 \sigma$, mínimo de eventos 10 e ruído de fundo $0.05 \%$. A legenda presente no gráfico da fonte Sculptor se aplica para todos os outros gráficos.

Figura 6.10 -Curvas de seção de choque de aniquilação por massa para as 18 fontes estudadas com duas configurações diferentes: significância $3 \sigma$, mínimo de eventos 7 e ruído de fundo $0.03 \%$ e significância $5 \sigma$, mínimo de eventos 10 e ruído de fundo $0.05 \%$. A legenda presente no gráfico da fonte Sculptor se aplica para todos os outros gráficos.

Figura 6.11 -Curvas de seção de choque de aniquilação por massa para as 18 fontes estudadas com duas configurações diferentes: significância $3 \sigma$, mínimo de eventos 7 e ruído de fundo $0.03 \%$ e significância $5 \sigma$, mínimo de eventos 10 e ruído de fundo $0.05 \%$. A legenda presente no gráfico da fonte Sculptor se aplica para todos os outros gráficos.

Figura 6.12 -Comparação das sensitividades referentes ao CTA (em magenta) e ao FERMI-LAT (verde). A curva referente ao CTA foi calculada com significância $3 \sigma$ e com $50 \mathrm{~h}$ de tempo de observação.

Figura 6.13 -Curvas de seção de choque de aniquilação por massa para as 18 fontes. 96 
Figura 6.14 -Curvas de seção de choque de aniquilação por massa para as 18 fontes. 97

Figura 6.15 -Curvas de seção de choque de aniquilação por massa para as 18 fontes. 98 



\section{LISTA DE TABELAS}

Tabela 5.1 - Propriedades das galáxias Draco e Segue 1 onde $d$ é a distância da galáxia à Terra, $M_{v}$ é a magnitude absoluta, $L$ corresponde à luminosidade em unidade de luminosidade do sol, $r_{h}$ é o raio half-light (distância do centro da galáxia até metade de seu raio luminoso) e $\frac{M}{L}$, a razão massa-luminosidade de cada uma. . . . . . . . 64

Tabela 5.2 - Valores obtidos com a implementação do algoritmo.

Tabela 5.3 - Valores do limite superior da seção de choque calculados pela implementação dos canais $\tau^{+} \tau^{-}$e $W^{+} W^{-}$. Erros calculados por propagação de erro. . . . . . . . . 69

Tabela 5.4 - Valores de limite de fluxo diferencial e integral. . . . . . . . . . . . . 70

Tabela 5.5 - Valores do fator $\tilde{J}$ para as galáxias Draco e Segue I. . . . . . . . . . . 71

Tabela 5.6 - Valores obtidos para o limite superior da seção de choque com a implementação do algoritmo para a galáxia Draco. . . . . . . . . . . . . . . 73

Tabela 5.7 - Valores obtidos para o limite superior da seção de choque com a implementação do algoritmo para a galáxia Segue I. . . . . . . . . . . . . . 73

Tabela 6.1 - Fatores astrofísicos $J$ correspondentes a cada fonte.

Tabela 6.2 - Valores dos parâmetros do polinômio de terceiro grau para cada curva correspondente a uma dada massa.

Tabela 6.3 - Valores de massa $m_{\chi}(\mathrm{TeV})$ e fluxo integral mínimo da fonte Sculptor $\Phi \mathrm{cm}^{-2} \mathrm{~s}^{-1}$.

Tabela 6.4 - Valores de seção de choque e respectiva massa para as fontes Sculptor, Ursa Minor e Segue I.

Tabela 6.5 - Fontes estudadas, seus fatores astrofísicos $J$ e respectivos erros.

Tabela 6.6 - Fontes e seus nomes por catálogo. . . . . . . . . . . . . . . . . . . . . . 90

Tabela 6.7 - Valores dos pontos obtidos para as galáxias presentes nas figuras 6.9, 6.10 e 6.11. . 99

Tabela 6.8 - Valores mínimos de massa por galáxia possíveis de serem obtidos pelo sinal captado pelo CTA. 



\section{SUMÁRIO}

1 Introdução

2 Matéria Escura $\quad 27$

2.1 Introdução histórica . . . . . . . . . . . . . . . . . . . . . . . . . . . . . . 27

2.2 Evidências sobre a existência de Matéria Escura . . . . . . . . . . . . . . . . . . . 28

2.2.1 Curvas de Rotação . . . . . . . . . . . . . . . . . . . . . . . . . . . . . . . . 28

2.2.2 Lentes Gravitacionais . . . . . . . . . . . . . . . . . . . . . . . . . . 30

2.2.3 Evidências cosmológicas . . . . . . . . . . . . . . . . . . . . . . . . . . 31

2.3 Perfis de Densidade . . . . . . . . . . . . . . . . . . . . . . . . . 32

2.3.1 Parametrização do perfil de densidade . . . . . . . . . . . . . . . . . . 32

2.3.2 Navarro - Frenk - White (NFW) . . . . . . . . . . . . . 33

2.3 .3 Hernquist . . . . . . . . . . . . . . . . . . . . . . . . . . . . 33

2.3 .4 Einasto . . . . . . . . . . . . . . . . . . . . . . . . . . . 34

2.4 Candidatos a Matéria Escura . . . . . . . . . . . . . . . . . . . . . . . . . . . 35

2.4.1 WIMP - SUSY . . . . . . . . . . . . . . . . . . . . . . . . . . . . . 35

2.4.2 Neutrinos Estéreis . . . . . . . . . . . . . . . . . . . . . . . . . . . . . 37

2.4 .3 Axions . . . . . . . . . . . . . . . . . . . . . . . . . . . . . . . . . 37

2.5 Possíveis Fontes Astrofísicas de Matéria Escura . . . . . . . . . . . . . . . . . . . . . 38

2.6 Detecção de Matéria Escura . . . . . . . . . . . . . . . . . . . . . . . 40

2.6.1 Mecanismos de interação . . . . . . . . . . . . . . . . . . . . . . . . 41

2.6.2 Produção em Aceleradores . . . . . . . . . . . . . . . . . . . . . . . . . . 41

2.6.3 Detecção Direta . . . . . . . . . . . . . . . . . . . . . . . . . . . . . . . . . . . 42

2.6 .4 Detecção Indireta . . . . . . . . . . . . . . . . . . . . . . . . . . . 43 
3 Detecção de matéria escura por telescópios de astronomia gama

3.1 Fluxo de raios gama . . . . . . . . . . . . . . . . . . . . . . 45

3.1 .1 Fator astrofísico $J \ldots \ldots \ldots \ldots$. . . . . . . . . . . . . . . . . . . . . . . . .

3.1.2 Contribuição de física de partículas para a geração de raios gama . . . . . . . . . . 49

4 Cherenkov Telescope Array - CTA 53

4.1 Telescópios Cherenkov . . . . . . . . . . . . . . . . . . . . . . . . . 53

4.2 Sensitividade . . . . . . . . . . . . . . . . . . . . . . . 54

4.3 Estudo da matéria escura pelo CTA . . . . . . . . . . . . . . . . . 58

5 Cálculo de limites na seção de choque de aniquilição 63

5.1 Estudo referente à análise do MAGIC $\ldots \ldots \ldots 4$

5.1.1 Fluxo de raios gama e fator astrofísico $J \ldots \ldots \ldots$

5.1.2 Taxa de produção de raios gama por aniquilação de partículas . . . . . . . . . . . 66

5.1 .3 Implementação . . . . . . . . . . . . . . . . . . . . . . . . . . 66

5.2 Estudo referente a análise do VERITAS . . . . . . . . . . . . . . . . . . . . . 69

5.2.1 Fluxo de raios gama e fator astrofísico J . . . . . . . . . . . . . . . . 69

5.2.2 Taxa de produção de raios gama por aniquilação de partículas . . . . . . . . . . 71

5.3 Conclusão sobre as análises . . . . . . . . . . . . . . . . . . . . . . . . . . . 74

6 Previsão do limite da seção de choque de aniquilação medido pelo CTA 77

6.1 Programa de Simulação e Primeiros Resultados . . . . . . . . . . . . . . . . . . 78

6.2 Resultados com fontes estudadas pelo FERMI-LAT . . . . . . . . . . . . . . . . 84

6.2 .1 Resultados com significância $3 \sigma$ e $5 \sigma \ldots$. . . . . . . . . . . . . . . 86

6.2.2 Análise da potencialidade do CTA no estudo de fontes medidas pelo FERMI-LAT . 94

$\begin{array}{lll}7 & \text { Conclusões } & 103\end{array}$

7.1 Cálculo de limites superiores na seção de choque de aniquilação . . . . . . . . . . 103

7.2 Previsão do limite superior da seção de choque de aniquilação medido pelo CTA . . 104 
7.3 Conclusões Gerais .

REFERÊNCIAS 



\section{Introdução}

Entre as maiores questões em aberto da ciência moderna estão os problemas relacionados à astrofísica de partículas. Por ser a interface entre a dinânima das estruturas do Universo e a física de seus constituintes mais intrínsecos, a astrofísica de partículas acaba por englobar alguns dos problemas mais populares atualmente. Dentre estes podemos citar a assimetria matéria/antimatéria, ondas gravitacionais, campos magnéticos e matéria escura.

Ao longo do século $X X$ até os dias atuais, evidências para a existência de matéria escura foram acumuladas (1-4) de forma que pudemos modelar um cenário convincente de que algo está em falta quando observamos estruturas do Universo. Essas evidências estão extremamente entrelaçadas com efeitos gravitacionais da matéria escura sobre a matéria bariônica sem qualquer resquício de uma interação eletromagnética, fato que resultou em seu nome. No âmbito dos efeitos gravitacionais, podemos medir curvas de rotação das galáxias, nas quais a existência de matéria escura é inferida pela dinâmica desses sistemas, e lentes gravitacionais, em que a curvatura da luz em campos gravitacionais intensos denuncia a presença de matéria escura.

É possível calcular, através das constantes cosmológicas e das teorias que modelam o Big Bang, a porcentagem dos constituintes do Universo. Atualmente atribuímos $\approx 72 \%$ a energia escura, $\approx 23 \%$ a matéria escura e apenas $\approx 5 \%$ a matéria comum. Essas frações de densidade são bastante alarmantes no contexto de conhecimento sobre o Universo, uma vez que conseguimos explicar apenas $\approx 5 \%$ dele. A falta de entendimento e de informação sobre a física do Universo é o combustível que precisamos para continuar a busca por respostas.

A fim de entender com o que lidamos quando nos referimos a matéria escura, vários modelos teóricos tomaram forma conforme obtínhamos dados experimentais. Porém, a natureza da partícula de matéria escura continua desconhecida. Entre esses modelos, alguns possuem maior destaque por não haver nenhuma observação que possa refutar sua existência e concordância com matéria escura, como exemplo as WIMPs (weakly interacting massive particles) (5) e dentro dessa classe específica, os neutralinos.

Entre as várias estratégias para detecção de um sinal proveniente de matéria escura temos uma forma de procura indireta da partícula. A detecção indireta foca no produto final da 
interação entre partículas de matéria escura. Nos modelos, é esperado que a aniquilação ou decaimento das partículas de matéria escura resultem em partículas secundárias e, posteriormente, raios gama. A detecção do fluxo de raios gama é de extrema importância porque no sinal agregamos informações sobre a física de partículas envolvida e sobre propriedades astrofísicas como a distribuição de matéria escura. Assim, podemos derivar propriedades da partícula, o que resulta em um passo para consolidar um possível modelo. Vários experimentos foram elaborados (como, por exemplo, MAGIC (6), H.E.S.S. (7) e VERITAS(8)) com potencialidade para detectar o possível fluxo de raios gama porém nenhum sinal irrefutável foi medido até o presente. Os resultados até agora são limites superiores nos valores de fluxo de raios gama possivelmente provenientes da matéria escura e, com esses limites, podemos inferir valores também limitantes para características da partícula como, por exemplo, o valor de sua seção de choque.

A futura contribuição do CTA para a detecção de fluxo de raios gama provenientes de matéria escura é esperada com grande expectativa pela comunidade científica. O CTA é um observatório em fase de construção previsto para operar em 2018-19. As características que o tornam foco das expectativas estão relacionadas com sua potencialidade. O intervalo de energia de operação atinge valores da ordem de $\approx 100 \mathrm{GeV}$ até $\approx 100 \mathrm{TeV}$, sendo possível atingir valores não alcançados com os experimentos anteriores. Em termos de sensitividade, esperam-se melhorias em uma ordem de grandeza.

Nesta dissertação, focamos na possibilidade de detecção do sinal de matéria escura proveniente da aniquilação de neutralinos pelo Observatório CTA. No capítulo 2, descrevemos as propriedades gerais de matéria escura. Abordamos desde as evidências de sua existência e os perfis que modelam sua distribuição até os principais modelos que envolvem a natureza das partículas, as possíveis fontes de detecção e os próprios métodos de detecção.

No capítulo 3 focamos no fluxo de raios gama a partir da aniquilação das partículas de matéria escura. Estudamos os detalhes que envolvem os termos do cálculo possibilitando um maior entendimento de todas as propriedades que resultam no fluxo de raios gama que podem atingir a Terra. A partir da familiarização com o cálculo do fluxo, no capítulo 4 descrevemos algumas propriedades do funcionamento dos telescópios de radiação Cherenkov.

No capítulo 5 apresentamos os primeiros resultados do cálculo do limite superior na seção de choque de aniquilação obtidos com dados do MAGIC e do VERITAS, a partir da elaboração de um algoritmo para o cálculo do limite superior da seção de choque de aniquilação do neutralino. No capítulo 6 consideramos as características do CTA a partir da implementação oficial de simulação, e então apresentamos os resultados de previsão de medidas de detecção 
indireta de matéria escura para diferentes fontes e diferentes configurações. Por fim, no capítulo 7 concluímos a dissertação com a interpretação dos resultados e o que estes representam para as expectativas de detecção indireta de matéria escura nos próximos anos. 


\section{Matéria Escura}

Matéria escura se refere a matéria presente no Universo que não observamos de forma usual, isto é, não possui interações eletromagnéticas que resultem na observação e caracterização de seus componentes. Sua existência é apenas comprovada por seus efeitos gravitacionais sobre matéria bariônica. Por não ter um comportamento de natureza conhecida, podemos afirmar que matéria escura não pode ser formada por partículas bariônicas.

Nesse capítulo discutiremos os aspectos gerais de matéria escura. Explicaremos o conceito de matéria escura através das evidências que comprovam sua existência, introduzindo alguns modelos e perfis de densidade bem estabelecidos. Por fim discutiremos algumas fontes em potencial e então os métodos de detecção atuais.

\subsection{Introdução histórica}

Os primeiros trabalhos implicando que o conteúdo luminoso de algumas galáxias não era massivo o bastante para explicar sua dinâmica foram realizados por volta de 1922 por Öpik (1) e de 1932 por Oort (2) mas somente com a contribuição de Fritz Zwicky (3) em 1933 o conceito de matéria escura foi adotado. Zwicky estudava o cluster de galáxias Coma e descobriu que a massa gravitacional deveria ser muito maior do que o esperado por sua luminosidade (aproximadamente 400 vezes maior). Esta estimativa foi inferida utilizando o teorema do Virial que fornece uma equação que relaciona a média temporal da energia cinética total de um sistema de $N$ partículas em equilíbrio com a média temporal da energia potencial (9) possibilitando comparar a massa dada pela dinânima do sistema com a massa que deveria corresponder à luminosidade do mesmo.

Porém, apenas em 1970, a hipótese de matéria escura foi consolidada quando Rubin e Ford (4) obtiveram medidas mais detalhadas de curvas de rotação. Suas análises foram as primeiras evidências de que uma grande quantidade de matéria não luminosa poderia existir além do limite visível de uma galáxia. O trabalho consistia em medir a velocidade de rotação 
dos gases em galáxias espirais com um espectrômetro mais efetivo do que os existentes até então, aumentando assim a precisão dos resultados anteriormente medidos por Zwicky.

Depois da confirmação da existência da matéria escura, houve uma crescente necessidade de um modelo que explicasse as observações. Dessa forma, surgiram vários experimentos com objetivo de consolidar um modelo de matéria escura em seu escopo de objetivos. Entre eles os telescópios de astronomia gama tem potencialidade para estudar uma faceta desse problema. Atualmente podemos limitar algumas características da possível partícula de matéria escura de forma bastante razoável, porém há um grande caminho a percorrer em termos tecnológicos antes que possamos determinar um modelo de extrema credibilidade.

\subsection{Evidências sobre a existência de Matéria Escura}

Para sustentar a existência de matéria escura e explicar as observações, vamos detalhar algumas evidências e exemplos que deram, ao longo dos anos, a certeza de que algo está faltando no modelo padrão das partículas elementares.

\subsubsection{Curvas de Rotação}

Historicamente, as primeiras evidências a apontarem a existência de matéria escura vieram de medidas relacionadas à velocidade de rotação de galáxias. A curva de rotação de uma galáxia corresponde à variação da velocidade orbital de estrelas ou nuvem de gases com relação a suas distâncias do centro. Nesses sistemas, assumindo que a única força atuante é a gravitacional causada pela matéria luminosa, é esperado um comportamento kepleriano, ou seja, que a velocidade seja inversamente proporcional à raiz quadrada da distância ao centro $\left(v \propto \frac{1}{r^{1 / 2}}\right)$. Uma curva com essa dependência tem um comportamento como indicado pela figura 2.1.

No entanto, as medidas de curvas de rotação mostram um comportamento diferente do esperado. Os dados experimentais mostram que conforme aumenta a distância ao centro da galáxia, o decaimento com $r^{1 / 2}$ não acontece e passamos a observar um comportamento constante (Figura 2.2).

A maioria das medidas de curva de rotação são feitas através de um espectrômetro observando o efeito Doppler (11) sobre uma linha de emissão do hidrogênio neutro no plano 
Figura 2.1 - Função $v=r^{-\frac{1}{2}}$ descritiva para um comportamento kepleriano, ou seja, tipicamente deveríamos ver uma versão parecida e suavizada para a velocidade da matéria que constitui uma galáxia.

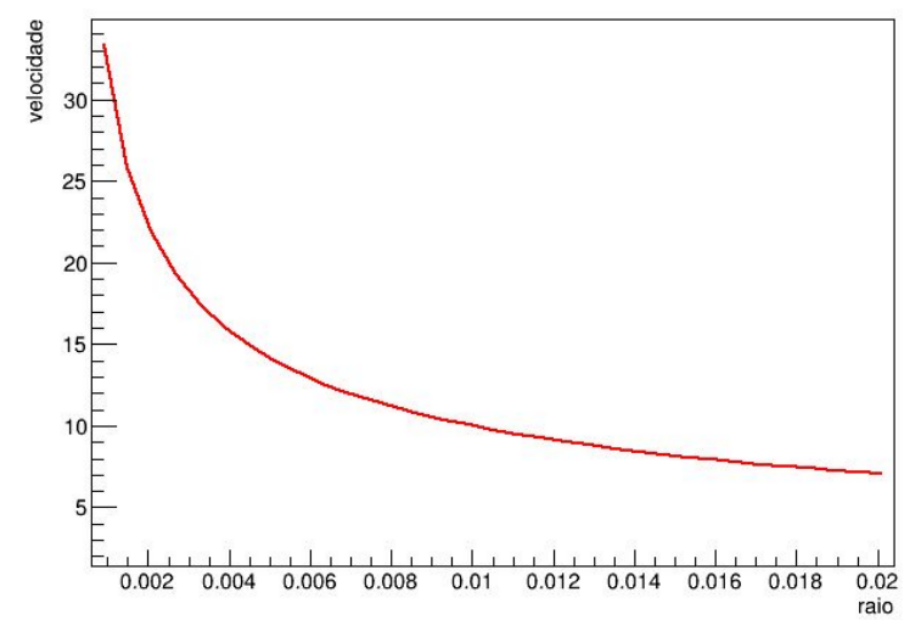

Fonte: Elaborada pela autora.

Figura 2.2 - Curva de rotação de NGC 6503 representada pela linha sólida com os dados experimentais. A linha tracejada representa a velocidade do conteúdo luminoso, a pontilhada representa a velocidade do gás, e a curva tracejada-pontilhada a velocidade inferida para a contribuição de matéria escura no sistema.

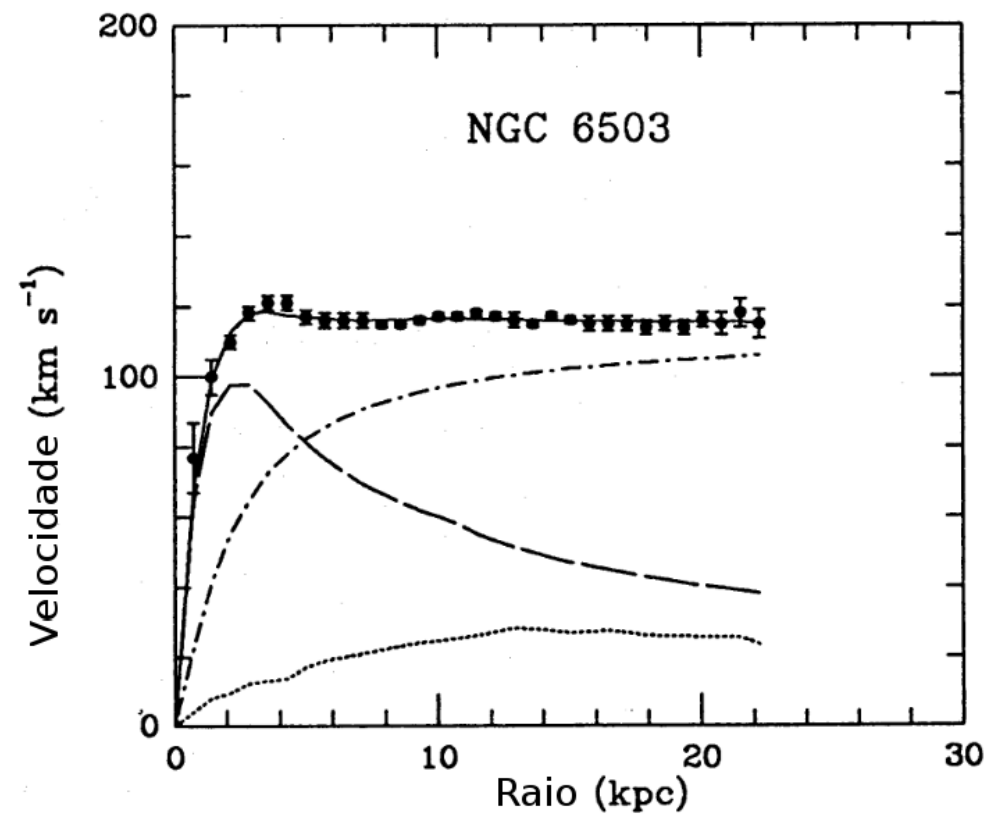

Fonte: Adaptada de BEGEMAN; BOEILS; SANDERS. (10) 
Figura 2.3 - Aglomerado de galáxias Bullet. Na esquerda, a imagem óptica mostra os contornos de matéria gravitacional inferidos por lentes gravitacionais. À direita, a imagem em raio-x do aglomerado mostra claramente a diferença de densidade de matéria visível e escura.
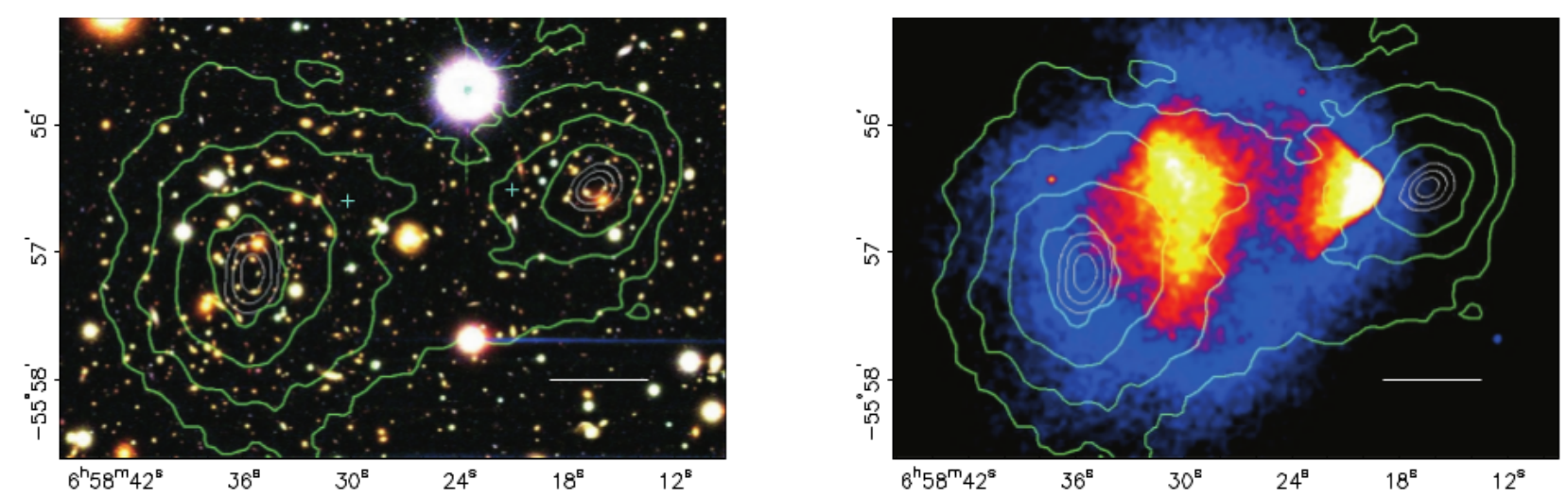

Fonte: CLOWE; BRADAC; GONZALEZ; MARKEVITCH; RANDALL; JONES; ZARITSKY. (13)

galáctico, inferindo assim sua velocidade orbital.

Uma vez que, quando nos afastamos do centro da galáxia, a dinâmica dos gases não pode ser consequência apenas da quantidade de matéria visível, a conclusão mais simples para esse comportamento é a existência de um halo de matéria escura que contribui para o efeito gravitacional sobre a matéria bariônica, impedindo que a velocidade orbital decaia conforme esperado.

\subsubsection{Lentes Gravitacionais}

Uma outra evidência da existência de matéria escura é proveniente do efeito chamado lentes gravitacionais. Pela teoria de Relatividade Geral de Einstein, concluímos, de maneira breve para este trabalho, que objetos massivos curvam o espaço-tempo, ou seja, quando observamos objetos distantes, a luz emitida é curvada para regiões mais massivas. A gravidade nesses sistemas tem um comportamento análogo ao causado por uma lente óptica, o que resulta no nome para o efeito. Dessa forma é possível que alguns fenômenos ocorram na imagem quando observamos um objeto astrofísico, como, por exemplo, múltiplas imagens do mesmo objeto, uma imagem distorcida, entre outros. Um exemplo bastante comum desse efeito foi primeiro observado no Observatório Chandra (12) no aglomerado de galáxias 1E 0657-56 ou Bullet Cluster que foi formado da colisão de outros dois aglomerados. (13. 14)

Na figura 2.3 podemos ver uma imagem óptica da colisão na esquerda e uma imagem de 
raio-x na direita. Os contornos verdes representam a densidade de massa inferida a partir do efeito de lentes gravitacionais. Na imagem de raio-x podemos ver que o conteúdo bariônico está deslocado das curvas de massa gravitacional, o que demonstra a existência de um tipo de matéria invisível que não interage da mesma forma que o conteúdo bariônico.

O deslocamento entre as regiões de alta densidade gravitacional e as regiões visíveis no raio-x acontece porque o gás quente desacelera a matéria bariônica durante a colisão como uma força de arraste mas não tem o mesmo efeito sobre a matéria escura porque esta apenas interage com o gás gravitacionalmente. Para que o efeito de lente gravitacional nesse aglomerado esteja de acordo com as previsões, é necessária a existência de matéria escura.

\subsubsection{Evidências cosmológicas}

Além das evidências de caráter astrofísico, temos algumas de caráter cosmológico que dão ainda mais suporte para a existência de matéria escura. O modelo do Big Bang é hoje a teoria mais bem sucedida para explicar as medidas cosmológicas mais precisas, tais como as flutuações da radiação cósmica de fundo. (15, 16)

A radiação cósmica de fundo (RCF) surgiu do desacoplamento da radiação com a matéria bariônica no período que chamamos de recombinação (elétrons e prótons formando átomos neutros), que tornou o Universo transparente para fótons. É caracterizada por ter um espectro de corpo negro com temperatura média de $T=2.7 \mathrm{~K}$ e com flutuações numa escala de $10^{-5}$ (17). Essas flutuações ou anisotropias são assinaturas de pertubações no potencial gravitacional durante a recombinação. As assinaturas que medimos são picos no espectro e a análise desses picos caracteriza vários parâmetros cosmológicos, como, por exemplo, geometria do Universo, proporção de matéria bariônica e de matéria escura presente no Universo, entre outros. Atualmente atribuímos aproximadamente $5 \%$ de matéria bariônica, $23 \%$ de matéria escura e $72 \%$ de energia escura à composição do Universo.

Outra evidência cosmológica importante se refere à formação de grandes estruturas do Universo (18). O tempo decorrido desde o Big Bang e os baixos valores de flutuação da radiação cósmica de fundo (RCF) não são suficientes para explicar a formação das estruturas de galáxias e aglomerados que existem hoje. Uma das explicações que evidenciam matéria escura é que sua existência teria provocado uma interação gravitacional mais potente do que previsto e começado a formação de estruturas antes do desacoplamento entre matéria comum e radiação, desta forma possibilitando os colapsos e a existências das estruturas como 
conhecemos hoje.

\subsection{Perfis de Densidade}

Se temos evidência sobre a existência de matéria escura podemos perguntar do que ela é feita e como é distribuída. Nesta seção discutimos a última questão e na seção seguinte faremos um resumo dos candidatos à matéria escura.

Um fator de extrema importância ao considerarmos possíveis modelos de matéria escura é o perfil de densidade. Estes descrevem a distribuição de matéria escura em torno de uma fonte astrofísíca específica. Para os cálculos envolvidos com a possível detecção de matéria escura, esses perfis são indispensáveis pois caracterizam a quantidade de matéria escura em função da distância $r$ do centro da fonte. Os perfis são obtidos a partir de simulações de muitos corpos para descrever a dinâmica das fontes astrofísicas a partir de dados experimentais como as curvas de rotação.

\subsubsection{Parametrização do perfil de densidade}

Como consequência da simulação de muitos corpos para a descrição do perfil, muitos modelos são gerados a partir de uma lei de potências. Por essa razão, alguns acabam por concordar com uma parametrização (19) da seguinte forma:

$$
\rho(r)=\rho_{0} \frac{a^{\delta}}{r^{\alpha}\left(a^{p}+r^{p}\right)^{\frac{\delta-\alpha}{p}}},
$$

onde $a$ representa o raio de escala que determina os comportamentos da função para $r \gg a$ e $r \ll a$. Os parâmetros $\alpha$ e $\delta$ são potências relacionadas ao centro e ao limite assintótico da função. Já o parâmetro $p$ está relacionado com a transição entre os comportamentos limitantes do perfil.

A parametrização descreve modelos chamados core-cusp models em inglês. Os primeiros modelos a serem elaborados para explicar a distribuição de matéria escura simulavam esferas isotérmicas de dois parâmetros: velocidade de dispersão e um raio de núcleo. Esse tipo de modelo permitia que as observações fossem diretamente comparadas com simulações de muitos corpos. A discussão acerca da validade do perfil isotérmico para halos de matéria escura 
provocou uma adaptação com uma curvatura logarítmica que descreve os modelos core-cusp. A parametrização é importante porque proporciona uma descrição geral para alguns modelos de perfis de densidade, o que nos aproxima de uma descrição geral desse comportamento.

\subsubsection{Navarro - Frenk - White (NFW)}

Um dos modelos de perfil que segue a parametrização segundo a equação ?? e é extremamente utilizado na literatura é o proposto por Navarro, Frenk and White (20). Um fator que contribui para sua popularidade é que o perfil foi elaborado a partir de sistemas em equilíbrio. Na parametrização, assumimos os valores $(\alpha, \delta, p)=(1,3,1)$ para o perfil NFW e obtemos a seguinte expressão:

$$
\rho(r)_{N F W}=\rho_{0}\left(\frac{r}{a}\right)^{-1}\left(1+\frac{r}{a}\right)^{-2} .
$$

Para os limites em relação ao raio de escala $a$, temos que:

$$
\begin{aligned}
& r \ll a \Rightarrow \rho(r)_{N F W} \propto r^{-1}, \\
& r \gg a \Rightarrow \rho(r)_{N F W} \propto r^{-3} .
\end{aligned}
$$

Esses limites representam um decaimento com $r^{-1}$ quando estamos considerando posições ainda dentro do halo de matéria escura. Para fora do halo, temos um decaimento mais rápido com $r^{-3}$, uma vez que para $r \gg a$, atingimos distâncias bem maiores que o halo.

A presença do perfil NFW na maioria das referências de detecção indireta evidencia a importância deste para a descrição de um modelo de distribuição de matéria escura. Podemos citar (21-25) entre várias outras referências que consideram o fator astrofísico $J$ derivado a partir do perfil NFW. Explicaremos o cálculo do fator astrofísico $J$ no capítulo 3 .

\subsubsection{Hernquist}

Elaborado anteriormente ao NFW, o perfil de Hernquist (26) difere do NFW apenas por um dos parâmetros. Adotamos aqui $(\alpha, \delta, p)=(1,4,1)$, o que resulta na seguinte expressão: 


$$
\rho(r)_{\text {Hernquist }}=\rho_{0}\left(\frac{r}{a}\right)^{-1}\left(1+\frac{r}{a}\right)^{-3} .
$$

Para $r \ll a$ o perfil se comporta da mesma forma que o perfil NFW tendo apenas uma diferença para $r \gg a$ onde a potência que regula $\rho(r)$ passa a ser -4 . Em termos de taxa de produção de raios gama, esses perfis não diferem muito; a principal diferença entre eles é a quantidade de massa que se considera envolvida no halo de matéria escura.

Como em termos gerais da distribuição o perfil Hernquist não difere de forma drástica do perfil NFW, sua utilização atualmente é majoritária em comparações com outros perfis.

\subsubsection{Einasto}

O último que vamos citar é o perfil Einasto (27). Este não segue a parametrização citada acima, mas tambem é bastante utilizado porque pode descrever perfis de distribuição de matéria escura tão bem quanto o perfil NFW.

Diferentemente dos perfis mencionados anteriormente e dos outros que seguem a parametrização da equação 2.1 o perfil Einasto foi elaborado com o valor de densidade de matéria escura para $r \rightarrow 0$, tendendo a zero. A eliminação da presença da divergência no valor de densidade no centro do perfil Einasto é razão suficiente para a escolha em utilizá-lo. Porém, as simulações atuais de muitos corpos não contêm dados suficientes para apontar diferenças significativas no centro dos perfis, fazendo com que o ajuste às fontes continue sendo a melhor forma de escolher um perfil. Além disso, o perfil Einasto possui três parâmetros livres $\left(\rho_{0}, \alpha, a\right)$ ao invés de dois descritos nos perfis da parametrização, tornando-o mais complexo para utilização em simulações.

Podemos expressar o perfil Einasto da seguinte forma:

$$
\rho(r)_{\text {Einasto }}=\rho_{0} \exp \left(-\frac{2}{\alpha}\left(\left(\frac{r}{a}\right)^{\alpha}-1\right)\right)
$$

Podemos citar como exemplo do uso do perfil Einasto as referências (28--31). Utilizamos a última como referência de dados para o cálculo do limite de seção de choque de aniquilação no capítulo 5 


\subsection{Candidatos a Matéria Escura}

Tendo descrito alguns exemplos de como mapear a morfologia de um halo de matéria escura, passamos agora para uma questão mais intrínseca do tópico: a descrição de modelos de possíveis partículas de matéria escura. Vamos citar nas próximas seções três modelos distintos do que essa partícula poderia ser. Há uma grande variedade de teorias plausíveis que poderíamos explorar, mas as apresentadas já nos proporcionam plena noção das possibilidades e variedades dos vários modelos.

Até agora, não sabemos a natureza dessas partículas, mas através das observações podemos limitar algumas características que a partícula deve ter. Nos itens abaixo, em negrito, temos características que independem do modelo de partícula. As outras características, menos gerais, descrevem alguns modelos como, por exemplo, partículas de matéria escura provenientes de Supersimetria.

\section{- A partícula precisa ser neutra, ou seja, não possuir carga elétrica;}

- Precisa ser estável em escalas cosmológicas para que ainda possa existir;

- Deve interagir apenas gravitacionalmente e fracamente;

- Sua massa precisa ser da ordem da máxima energia alcançada atualmente no acelerador LHC;

- Precisa seguir os valores de relic density estimados $\Omega \approx 0.23 \mathrm{e}\left\langle\sigma v>\approx 3 \cdot 10^{-26} \mathrm{~cm}^{3} \mathrm{~s}^{-1}\right.$. Esses valores determinam, respectivamente, a densidade de energia do Universo referente a presença de matéria escura e o valor da seção de choque necessária para que a partícula de matéria escura tenha sido produzida termalmente no Universo primordial.

\subsubsection{WIMP - SUSY}

Seguindo essas características, existem vários modelos teóricos que poderiam descrever a natureza da partícula. O primeiro modelo a ser citado é o que focamos neste trabalho de mestrado.

SUSY ou Supersimetria (32) é um modelo teórico de partículas que explica alguns problemas em aberto no Modelo Padrão, como o problema de hierarquia de massas. A teoria 
proporciona uma relação entre férmions e bósons a partir da afirmação de que toda partícula do modelo padrão possui um parceiro supersimétrico com diferença em spin de $\frac{1}{2}$. Dessa forma, férmions (spin semi-inteiro) estariam acompanhados de um bóson (spin inteiro).

As WIMPs (Weakly Interacting Massive Particles) são uma classe de partículas que existe dentro desse modelo teórico e que têm aspectos convincentes em termos de explicar a natureza da matéria escura. (5)

De acordo com o modelo, essas partículas estavam em equilíbrio térmico no Universo primordial até que a temperatura começou a diminuir drasticamente e o equilíbrio por fim fosse interrompido. Conforme a temperatura diminuía, e o Universo expandia, a densidade do gás de WIMPs também diminuía, reduzindo assim a probabilidade de aniquilação. Isso aconteceu porque a temperatura atingiu um valor menor do que a massa dessas partículas, fazendo com que a densidade fosse tão baixa que as WIMPs passaram a não mais colidir, e portanto não se aniquilar. Assim a densidade dessas partículas presentes no Universo se tornou constante.

O desacoplamento que resultou no valor constante sobre a densidade das WIMPs aconteceu quando a taxa de aniquilação destas se tornou menor do que a taxa de expansão do Universo $H$. A partir das relações de Friedmann (33), podemos escrever que:

$$
\Omega h^{2} \approx \frac{3 \cdot 10^{-27}}{<\sigma v>_{\text {aniquilação }}},
$$

onde $\Omega h^{2}$ está relacionado com a densidade de partículas WIMPs e $<\sigma v>_{\text {aniquilação é a seção }}$ de choque de aniquilação da partícula. Essa expressão regula os valores de densidade de matéria escura no Universo e também coloca uma referência como ponto inicial para caracterizar a seção de choque da partícula.

A densidade cosmológica dessas partículas assume o valor estimado quando comparamos a seção de choque de aniquilação com a seção de choque de escalas fracas. Essa proximidade de valores estimados ficou conhecida como "Milagre WIMP".

Dentro dessa classe, temos a partícula chamada neutralino. A razão por serem os neutralinos considerados favorito a constituírem matéria escura é porque possuem uma massa da ordem de $\mathrm{GeV}$ - TeV, uma seção de choque da ordem daquelas em interações fracas e devido a sua natureza física, poderem se aniquilar em partículas do modelo padrão. Todas essas razões fazem com que a hipótese de detecção seja bastante plausível. Esse modelo específico é o que utilizamos para concluir os resultados desse trabalho. 


\subsubsection{Neutrinos Estéreis}

Uma outra forma de abordar a natureza das partículas de matéria escura é apostar nos neutrinos como solução. Neutrinos ativos apenas interagem através da gravidade e pela força fraca, fazendo com que sejam invisíveis a fótons. Por terem uma massa pequena, com limite superior estimado em $0.28 \mathrm{eV}$ (34), neutrinos comuns não são bons candidatos a matéria escura porque são quentes demais (muito rápidos) para que possam formar toda a estrutura e massa esperada da matéria escura presente no Universo.

Os neutrinos estéreis são um tipo de neutrino que diferem dos neutrinos ativos principalmente porque não interagem fracamente, fazendo sua detecção ser ainda mais complicada. Com uma massa da ordem de $\mathrm{keV}$, a relação com a formação de grandes estruturas passa a ser possível. O que veríamos como forma de comprovar sua existência seria apenas uma assinatura, ou seja, um produto do decaimento de neutrinos estéreis em neutrinos ativos. Mesmo possuindo uma massa maior do que os neutrinos ativos, a energia resultante do decaimento ainda é baixa e por isso esperaríamos a assinatura na observação de uma linha do espectro de raio-x, por exemplo, de uma galáxia.

Um estudo recente envolvendo uma linha no espectro de raio-x de um cluster de galáxias considera neutrinos estéreis responsáveis pela emissão. Na referência (35) é considerada então a possibilidade de serem os neutrinos estéreis candidatos a matéria escura.

\subsubsection{Axions}

O último modelo que vamos apresentar são as chamadas partículas tipo áxion Axion-Like Particles - ALP. Essas partículas foram propostas a fim de explicar o problema Strong-CP da Cromodinâmica Quântica (36). Do modelo criado, podemos atribuir uma grande quantidade de matéria não bariônica aos ALP, o que os torna candidatos em potencial para matéria escura.

Na presença de campos magnéticos, as ALPs teoricamente podem se converter em fótons e vice-versa, e é a partir da mistura ALP-fóton que esperamos obter algum sinal da existência desses axions. Dentro do formalismo, existem algumas maneiras de identificar a partícula. $\mathrm{Na}$ figura 2.4 podemos ver os possíveis casos.

Os axions são considerados possíveis candidatos a matéria escura porque são neutros e apenas interagem fracamente. Se produzidos na quantidade correta, poderiam ser compo- 
Figura 2.4 - Possíveis conversões ALP-fóton que poderíamos identificar a partir da fonte até a Terra. As partículas $\gamma$ e a representam o fóton e o ALP respectivamente. $B_{C M I G}$ corresponde ao campo magnético intergaláctico, $B_{\text {fonte }}$ campo magnético da fonte e NGA representa o núcleo galático ativo que é a fonte em questão. $E B L$ representa o ruído de fundo extragalático (do inglês Extragalactic Background Light)

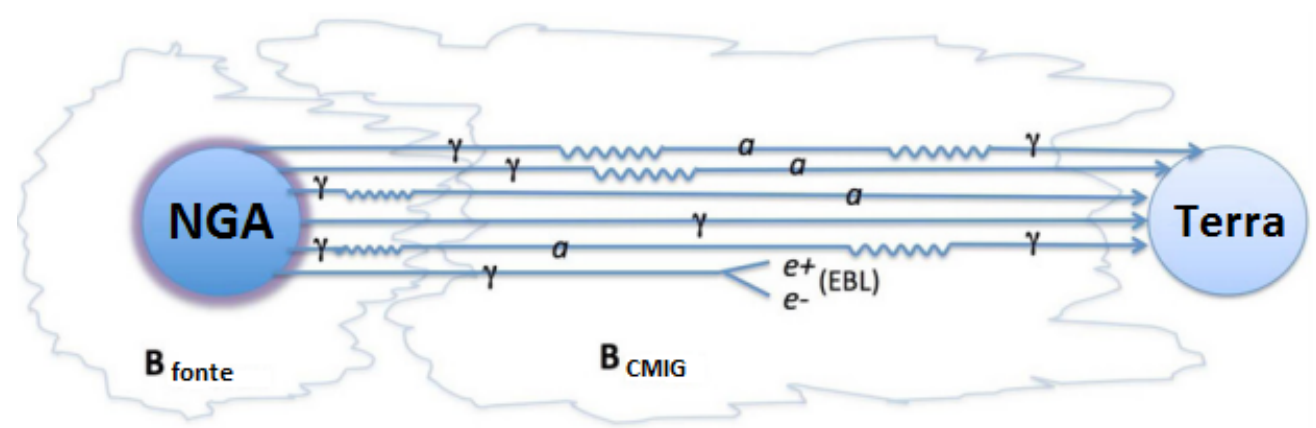

Fonte: Adaptada de SÁNCHEZ-CONDE; PANEQUE; BLOOM; PRADA; DOMÍNGUEZ. (37)

nentes de matéria escura. Um dos atrativos para o modelo dos axions é que, por terem sido propostos a fim de explicar um outro problema, se sua existência for confirmada, solucionaria duas questões atuais da área.

\subsection{Possíveis Fontes Astrofísicas de Matéria Escura}

Para estudar o comportamento de matéria escura no Universo, além dos possíveis modelos teóricos para a natureza da partícula, é preciso considerar os objetos astrofísicos que proporcionam maior potencialidade para o estudo de tal comportamento. Com uma variedade de objetos possíveis, devemos buscar fontes com uma alta densidade de matéria escura em relação a matéria bariônica. Isso pode ser deduzido a partir da razão massa-luminosidade $\frac{M}{L}$, que representa a razão de massa presente na fonte com sua luminosidade. Outro fator importante a ser considerado quando escolhemos uma fonte é a proporção do ruído de fundo no sinal que captamos no telescópio. O ruído de fundo pode ser proveniente de vários fatores como, por exemplo, a presença de outros objetos astrofísicos na região de observação.

Listamos algumas fontes possíveis de detecção de matéria escura abaixo, relacionando sua potencialidade com as características de cada uma. Nesta dissertação, focamos nas galáxias anãs esferoidais.

O fator astrofísico $J$ define a potencialidade de uma fonte para detecção de matéria escura. Explicaremos o comportamento desse fator no capítulo 3. 
O Centro Galáctico é uma fonte que tem atenção de vários experimentos porque em um primeiro momento, duas das principais características que devemos procurar em fontes para detectar matéria escura são cumpridas: é uma região próxima $(\sim 8.5 \mathrm{kpc})$ e em que se espera uma alta densidade de matéria escura. As desvantagens de considerar o Centro Galáctico residem no fato de que, apesar de suas qualidades, existe também uma alta densidade de outros objetos astrofísicos no plano galáctico que resultam em um sinal de emissão difuso, produto das interações hadrônicas dos raios cósmicos com nuvens moleculares gigantes. Outra razão para o ruído de fundo ser muito alto, é a presença da fonte HESS J1745-290 (38) que distorce, junto com a emissão difusa, a simulação do perfil de densidade de matéria escura.

Uma forma de contornar as adversidades da medida direcionada ao Centro Galáctico seria apontar para regiões fora do plano galático. Com a condição de que essas regiões estejam perto o bastante para detectar um fluxo de raios gama de matéria escura no halo da Via Láctea, essa estratégia proporciona a diminuição do sinal difuso proveniente dos objetos astrofísicos do plano galáctico. Baseando-se nessa técnica, recentemente o Observatório H.E.S.S. divulgou dados de $112 \mathrm{~h}$ de observação com uma distância angular entre $0.3^{\circ}$ e $1.0^{\circ}$ do Centro Galáctico, concluindo limites para a seção de choque de aniquilação $\langle\sigma v\rangle$ de matéria escura da ordem de $10^{-25} \mathrm{~cm}^{3} \mathrm{~s}^{-1}$. No modelo supõe-se que as partículas de matéria escura sejam WIMPs com massas acima de $100 \mathrm{GeV}$ (energia de limiar). (22)

Outro tipo de fonte importante de emissão de raios gama são as galáxias anãs esferoidais (em inglês Dwarf spheroidal galaxies - $d S p h$ ) do Grupo Local que são satélites orbitando a região da Via Láctea por sua força gravitacional. Essas fontes são importantes porque possuem condições muito favoráveis à detecção de matéria escura. As galáxias dSph possuem um alto valor da razão massa-luminosidade, $\left(\frac{M}{L} \propto 10^{2}\right)$ indicando uma forte concentração de matéria escura. Devido à falta de atividade de formação estelar e pouco material de raios cósmicos, as galáxias $d S p h$ possuem baixo ruído de fundo, o que aumenta a qualidade do sinal esperado. Por ter comparativamente baixa densidade de matéria bariônica, o halo de matéria escura também passa a ser facilmente simulado, uma vez que o conteúdo bariônico não interfere com o mapeamento do perfil de densidade.

Os melhores resultados de limite superior na seção de choque de aniquilação envolvendo galáxias dSph foram obtidos pelo Observatório FERMI-LAT (39). A figura 2.5 mostra o limite combinado de 25 galáxias derivado pelo Observatório em comparação com as curvas determinadas pelo Observatório H.E.S.S. do Centro Galáctico mencionado anteriormente.

Grandes sistemas ligados gravitacionalmente como aglomerados de galáxias também são considerados potenciais fontes para detecção de matéria escura. Com massas em torno de 
Figura 2.5 - Curvas de limites superiores da seção de choque de aniquilação determinada por diferentes experimentos. A curva preta sólida representa o limite superior combinado de 25 galáxias determinada pelo FERMI-LAT. A curva preta tracejada representa apenas - limite para a galáxia Segue 1. As curvas vermelha e a azul correspondem à curva determinada pelo H.E.S.S. da seção de choque no Centro Galáctico e da galáxia Segue 1 determinada pelo VERITAS, respectivamente.

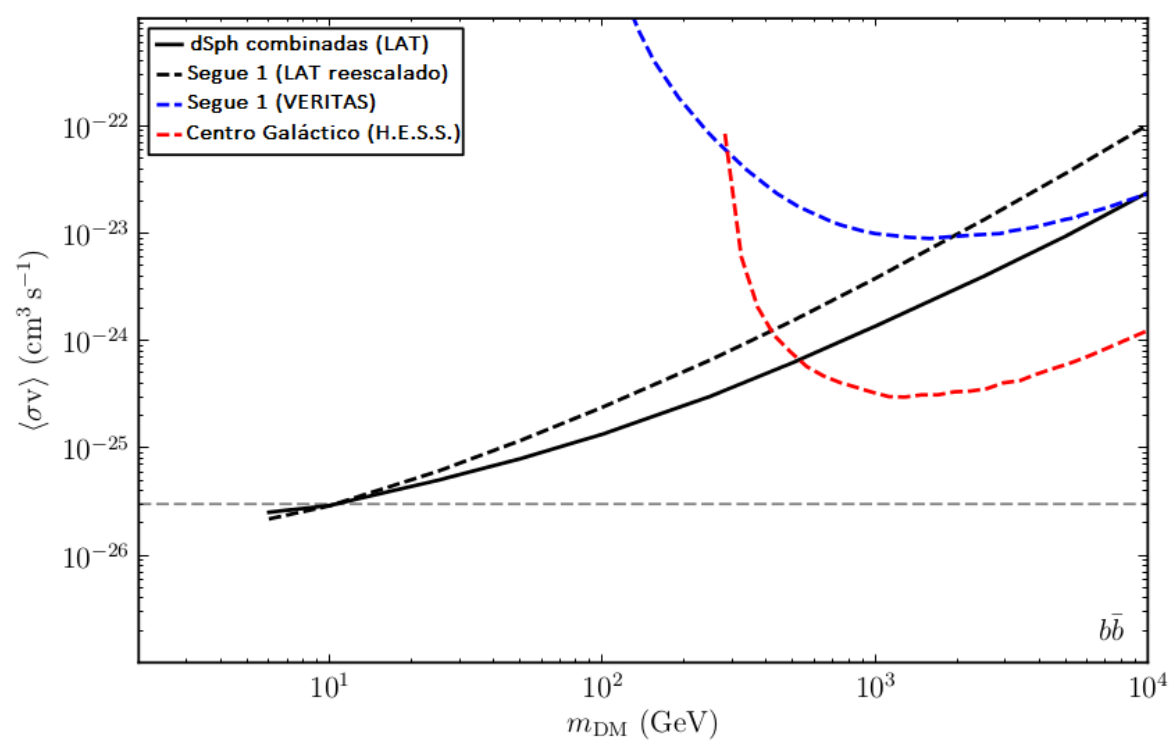

Fonte: Adaptada de ACKERMANN. (23)

$10^{14}-10^{15} M_{\odot}$, sua composição de matéria escura pode ser de $80 \%$ desse número, criando um forte potencial de detecção de raios gama originários da aniquilação de partículas de matéria escura. Por outro lado, a composição dos aglomerados não se restringe a matéria escura, portanto também há uma contribuição no sinal de raios gama que não pertence à aniquilação dessas partículas. Além da possibilidade de existirem Núcleos Galácticos Ativos, os raios cósmicos que interagem com o meio interestelar dos aglomerados também podem emitir raios gama e essa contribuição para o sinal detectado não é pequena. Isso faz com que o ruído de fundo da medida seja alto e difícil de contornar.

\subsection{Detecção de Matéria Escura}

Nessa seção focaremos nos mecanismos de interação das partículas relacionando-os ao meios de detecção hoje propostos. 


\subsubsection{Mecanismos de interação}

Para que possamos explicar os experimentos possíveis de detecção, precisamos primeiro ressaltar os mecanismos de interação das partículas. Assume-se que a natureza das interações de partículas de matéria escura são de força fraca por razões como o "Milagre WIMP". Para representar a partícula de matéria escura utilizaremos a letra $\chi$.

1. Aniquilação: processo descrito por $\chi+\chi \rightarrow \mathrm{MP}+\mathrm{MP}$, onde MP representa uma partícula do Modelo Padrão. Considera-se que partículas de matéria escura possam se aniquilar em fótons, neutrinos, elétrons, prótons e suas antipartículas. Para o neutralino também são considerados canais de aniquilação principais através das seguintes partículas secundárias: $b \bar{b}, t \bar{t}, \mu^{+} \mu^{-}, \tau^{+} \tau^{-}, W^{+} W^{-}$e $Z Z$. Nesta dissertação estudamos o processo de aniquilação de neutralinos;

2. Decaimento: processo descrito por $\chi \rightarrow \mathrm{MP}+\mathrm{MP}$. Consideram-se possíveis os mesmos canais e produtos resultantes da aniquilação, com a diferença de que se a partícula resultante não for estável, o processo de decaimento continua;

3. Produção: processo descrito por $\mathrm{MP}+\mathrm{MP} \rightarrow \chi+\chi$. Nos experimentos envolvendo produção de matéria escura procura-se observar o recuo das partículas do Modelo Padrão e deduzir a presença de matéria escura analisando a energia de recuo envolvida.

A figura 2.6 apresenta um esquema mostrando os métodos de detecção envolvendo esses processos.

\subsubsection{Produção em Aceleradores}

A detecção de matéria escura através de sua produção em aceleradores de partículas traz uma nova perspectiva em relação a matéria escura. Teríamos informações suficientes para explicar sua natureza e propriedades como massa, seção de choque, entre outras. Se considerarmos um modelo de partícula WIMP, a produção seria inferida pelo resultado da colisão.

Para uma partícula WIMP, a assinatura que procuramos ver está na criação de partículas supersimétricas que então decairiam em jatos de quarks e gluons e pares de neutrinos. Esses 
Figura 2.6 - Representação dos métodos de detecção e processos de interação de partículas de matéria escura. A partícula entitulada $\chi$ representa a partícula de matéria escura e a entitulada $M P$, partículas do Modelo Padrão

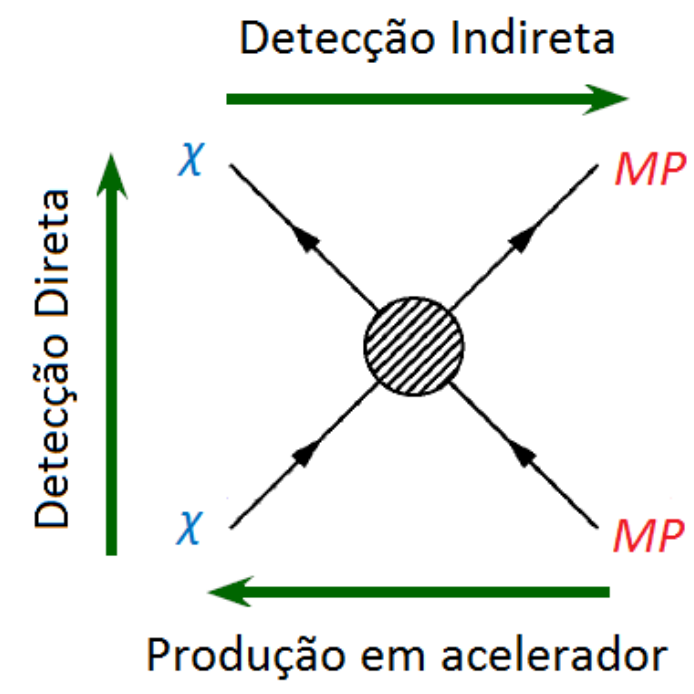

Fonte: Adaptada de MAX PLANCK SOCIETY. (40)

eventos podem ser detectados através da energia transversal faltante ( missing transverse energy - MTE). Por conservação de momento e energia, podemos calcular o quanto de energia o acelerador deve registrar e então, quando uma certa quantidade de energia não é detectada, atribuímos a uma partícula que também não foi detectada no experimento, ou seja, a partícula carrega a quantidade faltante.

Até agora, detectores como ATLAS e CMS não tiveram sucesso, mas há uma boa perspectiva para os próximos anos quando o LHC começar a operar em sua energia máxima de $14 \mathrm{TeV}$. (41)

\subsubsection{Detecção Direta}

Uma outra forma de abordar o problema é através da detecção direta da partícula. Pelo esquema da figura 2.6 podemos ver que a detecção direta está relacionada com a interação de partícula de matéria escura com uma partícula do Modelo Padrão.

O que queremos medir com esse tipo de abordagem é um desvio no núcleo indicando o espalhamento da partícula de matéria escura por uma partícula bariônica. Se considerarmos novamente as WIMPs, deveríamos ter na Terra um fluxo da ordem de $10^{5} \mathrm{~cm}^{-2} \mathrm{~s}^{-1}$ para uma partícula de $m_{\chi}=100 \mathrm{GeV}$ possibilitando que uma fração delas interagisse dessa forma com 
a matéria bariônica (5).

Os experimentos com enfâse em detecção direta têm um desafio grande em relação a controle de ruído de fundo de raios cósmicos porque se espera que a taxa de interações dessa natureza seja extremamente baixa. Alguns exemplos de experimentos que trabalharam com detecção direta de matéria escura são XENON (42), DAMA/LIBRA (43) e CDMS (44).

\subsubsection{Detecção Indireta}

A última forma de detecção a mencionar é a indireta. Esse tipo de detecção visa observar o produto da interação de partículas $\chi \operatorname{com} \chi$, ou seja, não observamos a partícula em si, mas sim o resultado de sua interação.

Esse trabalho de mestrado é focado nesse método específico de detecção, mais precisamente pelo processo de aniquilação de neutralinos. O próximo capítulo é dedicado aos detalhes envolvidos no método. 


\section{Detecção de matéria escura por telescópios de astronomia gama}

Dentro dessa categoria de detecção podemos considerar os processos de aniquilição e decaimento resultando partículas do Modelo Padrão que são possíveis de detectar. O sinal que procuramos vem de partículas como elétrons, prótons, fótons, neutrinos e suas respectivas antipartículas. Dada a variedade de produtos finais que podemos obter, a dificuldade desse método é identificar o ruído de fundo astrofísico nas medidas, ou seja, diferenciar os eventos originários de matéria escura dos outros possíveis eventos.

Alguns resultados interessantes tem sido publicados ((21, 45, 46), (30) entre outros) utilizando medidas de telescópio de astronomia gama a fim de limitar características da partícula de matéria escura através da detecção indireta. Um cenário geral ao longo dos anos dos limites estimados de seção de choque da possível partícula pode ser observado na figura 3.1.

\subsection{Fluxo de raios gama}

Como mencionado anteriormente, este trabalho de mestrado é focado na detecção indireta de partículas de matéria escura, mais precisamente pela aniquilação de neutralinos que se transformam em partículas secundárias e que então proporcionam como produto final um fluxo de raios gama. A identificação desses produtos permite restringir algumas propriedades da partícula, como por exemplo seção de choque e massa, e a partir dessas características, melhorar ou concretizar um modelo específico que acomode todos os dados experimentais.

Uma característica de raios gama que facilita a identificação das fontes é o fato de que esses raios não são defletidos pelo campo magnético cósmico, o que significa que o fluxo de raios gama detectado traça diretamente a sua origem. No contexto de astronomia de raios gama, a expressão para o fluxo diferencial é dada por: 
Figura 3.1 - Limites do valor da seção de choque ao longo dos anos determinados pelos experimentos: na ordem da legenda, (47), (48), (29), (49), (50), (51), (30), (22), (52), (53), (54), (55), (45), (31). Estes incluem galáxias $d S p h$, centro galáctico e linhas monocromáticas de detecção. Em vermelho e azul consideramos respectivamente os intrumentos espaciais e os terrestres que detectam aniquilação de matéria escura em $b \bar{b}$, e em verde os que detectam uma linha monocromática $\gamma \gamma$. O verde claro e o vermelho claro são detecções com um tempo de exposição 100 vezes maior do que os 10 anos do FERMI-LAT.

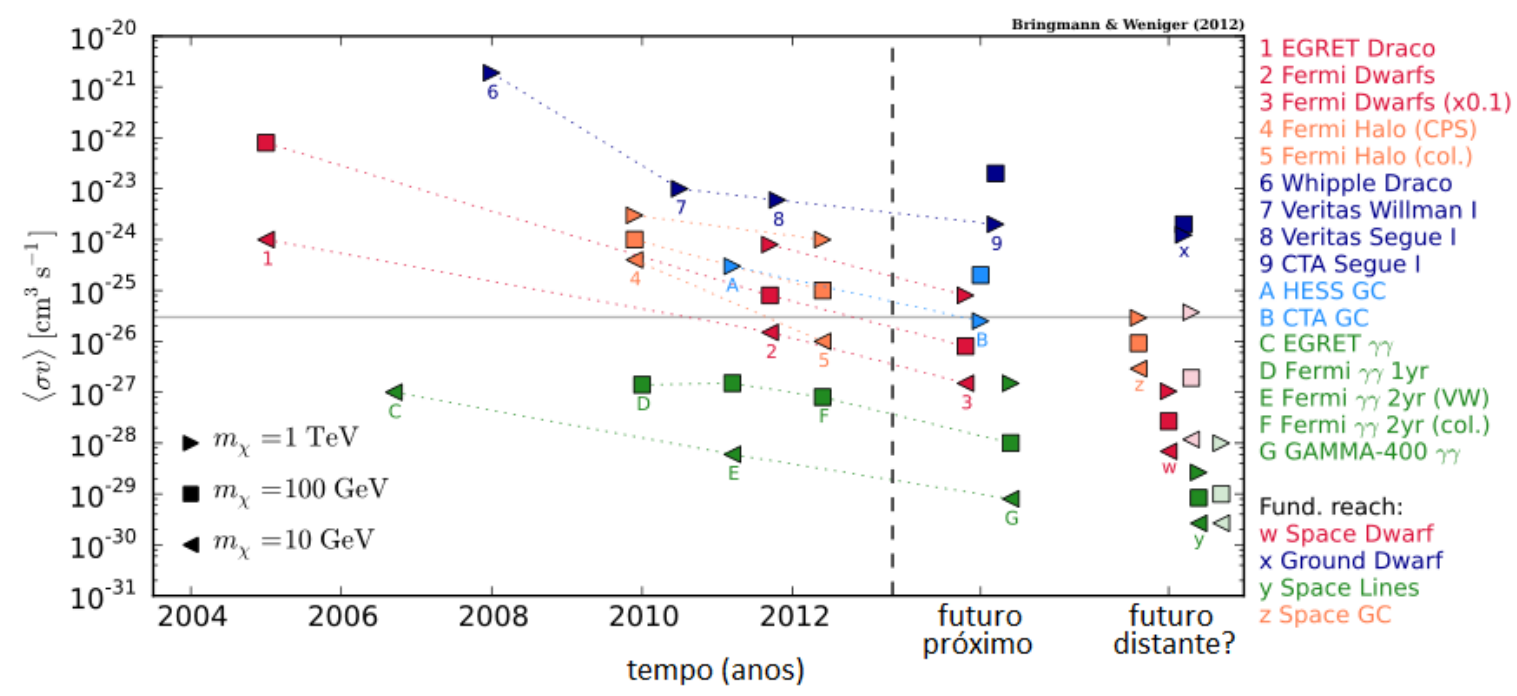

Fonte: Adaptada de BRINGMANN; WENIGER. (56)

$$
\frac{\mathrm{d} \Phi(\Delta \Omega)}{\mathrm{d} E}=\frac{\mathrm{d} \Phi^{P P}}{\mathrm{~d} E} \times J(\Delta \Omega)
$$

Note que podemos separar a expressão em duas contribuições:

1. O termo $J$ é chamado de fator astrofísico da observação. É nesse termo que incluímos as informações astrofísicas que influenciam o sinal detectado, ou seja, a contribuição do perfil de densidade de matéria escura $\rho(r)$.

2. O segundo termo $\frac{\mathrm{d} \Phi^{P P}}{\mathrm{~d} E}$, onde $P P$ se refere a Particle Physics, envolve propriedades do modelo de física de partículas que estamos considerando. É nesse termo que incluímos a massa da partícula, sua seção de choque e a taxa de produção de raios gama por interação. 


\subsubsection{Fator astrofísico $J$}

Quando consideramos o fluxo de raios gama que chega no observatório, precisamos levar em conta todas os fatores que podem influenciar o resultado final no instrumento de detecção. O fator astrofísico $J$ nada mais é do que uma medida de detectabilidade da fonte por um telescópio, ou seja, quanto maior o $J$, maior potencial de detecção a fonte em questão tem. Como na expressão do cálculo de $J$ temos que considerar a linha de visão (do inglês line of sight - los) e o ângulo sólido referente à fonte, as informações do telescópio se transferem para o valor final de $J$. Por essa razão, para uma mesma fonte e um mesmo perfil de densidade de matéria escura, podemos obter diferentes valores de $J$ se estivermos considerando diferentes observatórios.

Para o fator astrofísico de aniquilação, temos a seguinte expressão:

$$
J_{a n}(\Delta \Omega)=\int_{\Delta \Omega} \int_{l o s} \rho^{2}(l, \Omega) \mathrm{d} l \mathrm{~d} \Omega
$$

onde $\rho(r)$ é o perfil de densidade de matéria escura.

Vamos derivar essa expressão para um resultado dependente apenas de $r$, ou seja, apenas do tamanho da fonte.

Se considerarmos que a fonte tem emissão isotrópica, o que vemos é apenas uma fração de toda sua emissão, assim podemos escrever que:

$$
J_{a n}(\Delta \Omega)=\frac{1}{4 \pi D^{2}} \int \rho^{2}(\vec{x}) \mathrm{d} \vec{x},
$$

onde $D$ é a distância do observador até a fonte e $4 \pi D^{2}$ a casca esférica de raio $D$. Assim, a divisão por $4 \pi D^{2}$ implica que estamos considerando apenas o fluxo de raios gama que chega até o observador na Terra.

Analisando o aspecto tridimensional da expressão, devemos integrar $\rho(\vec{x})$ em $\mathrm{d} \vec{x}$. Então, em coordenadas esféricas,

$$
J_{a n}=\frac{1}{4 \pi D^{2}} \int_{0}^{2 \pi} \int_{0}^{\pi} \int_{0}^{R} \rho^{2}(r) r^{2} \sin \theta \mathrm{d} \theta \mathrm{d} \phi \mathrm{d} r
$$

Note que $\sin (\theta) \mathrm{d} \theta \mathrm{d} \phi$ é o elemento de ângulo sólido, logo 
Figura 3.2 - Representação do cone de visualização de uma fonte extensa com abertura $\theta$. A esfera roxa representa a fonte com extensão de raio $r$ e distância até o observador $D$.

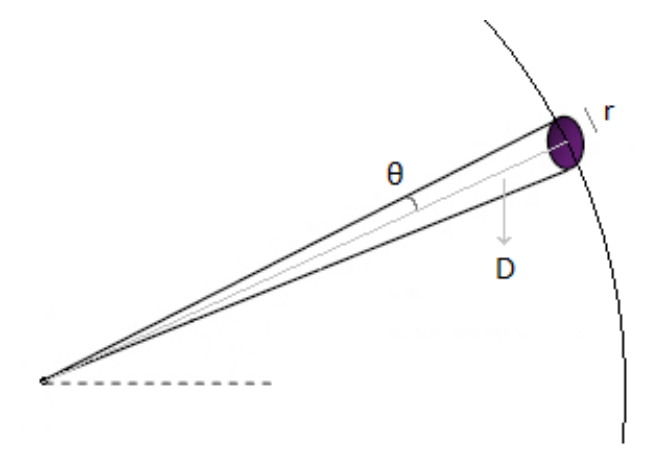

Fonte: Adaptada de SPIE MEMBERS. (57)

$$
J_{a n}=\frac{1}{4 \pi D^{2}} \int \mathrm{d} \Omega \int_{0}^{R} \rho^{2}(r) r^{2} \mathrm{~d} r
$$

Como $\int \mathrm{d} \Omega$ é avaliada em toda casca esférica de raio $r$,

$$
J_{a n}=\frac{1}{4 \pi D^{2}} 4 \pi \int_{0}^{R} \rho^{2}(r) r^{2} \mathrm{~d} r J_{a n}=\frac{1}{D^{2}} \int_{0}^{R} \rho^{2}(r) r^{2} \mathrm{~d} r .
$$

Essa simplificação da expressão de $J$ para uma dependência apenas com $r$ é bastante utilizada nos casos em que as fontes estão distantes o bastante para que possam ser consideradas fontes pontuais. Nos casos em que escolhemos fontes mais próximas, as chamadas PSF (Point Spread Function) são consideradas no sinal recebido. Essas funções indicam o poder de resolução do telescópio, isto é, quando temos uma fonte extensa, a resolução de sua imagem passa a ser um fator importante.

Para fins didáticos, a simplificação é bastante valiosa porque podemos ver exatamente como a escolha mais apropriada do perfil de densidade $\rho(r)$ influencia no resultado final da expressão de fluxo.

Em algumas referências o fator $4 \pi$ de $J$ é omitido e considerado no termo relacionado à física de partículas. Esses detalhes dependem da convenção que se escolhe utilizar.

Para fontes extensas, é preciso considerar a integração no ângulo sólido $\delta \Omega$. Para isso basta analisarmos o ângulo de abertura do cone que envolve a fonte extensa, veja figura 3.2. Assim, a integração no ângulo sólido se torna: 


$$
\begin{aligned}
\int \mathrm{d} \Omega & =\int_{0}^{2 \pi} \int_{0}^{\theta} \sin \left(\theta^{\prime}\right) \mathrm{d} \phi \mathrm{d} \theta^{\prime} \\
& =2 \pi \int_{0}^{\theta} \sin \left(\theta^{\prime}\right) \mathrm{d} \theta^{\prime} \\
& =2 \pi\left[-\left.\cos (\theta)\right|_{0} ^{\theta}\right] \\
& =2 \pi(1-\cos (\theta)) .
\end{aligned}
$$

Dessa forma, na equação 3.5 podemos substituir o valor da integral do ângulo sólido pela expressão dependente do ângulo de abertura do cone $\theta$. A expressão resultante inclui fontes extensas com uma dependência apenas com o ângulo de abertura do telescópio. É comum encontrar nas referências o valor de $\theta$ utilizado pelo telescópio para uma medida, possibilitando modelar os perfis de densidade a partir dessa informação.

\subsubsection{Contribuição de física de partículas para a geração de raios gama}

Na seção anterior, definimos a contribuição das características astrofísicas para a geração de raios gama a partir do termo $J$. A contribuição dos modelos teóricos e a física de partículas envolvida no processo estão relacionados com o primeiro termo da equação 3.1 representado por $\frac{\mathrm{d} \Phi^{P P}}{\mathrm{~d} E}$.

Podemos expressar o termo da seguinte forma:

$$
\frac{\mathrm{d} \Phi^{P P}}{\mathrm{~d} E}=\frac{<\sigma v>}{2 m_{\chi}^{2}} \frac{\mathrm{d} N_{\gamma}}{\mathrm{d} E}
$$

onde $m_{\chi}$ corresponde à massa da partícula de matéria escura, $\langle\sigma v\rangle$ corresponde à média da seção de choque de aniquilação pela distribuição de velocidades e $\frac{\mathrm{d} N_{\gamma}}{\mathrm{d} E}$ é a taxa de produção de raios gama por interação $\chi \operatorname{com} \chi$. Referimo-nos ao termo $\langle\sigma v>$ apenas como seção de choque de aniquilação omitindo a média com a distribuição de velocidades para facilitar a identificação do termo com outras referências.

Como mencionado anteriormente na seção 2.4.1, de acordo com o modelo vigente do Big Bang, a densidade de partículas WIMP assumiu um valor constante após a época de recombinação do Universo. Estima-se que o valor natural da seção de choque considerando essa época tenha sido de $\langle\sigma v\rangle \sim 3 \cdot 10^{-26} \mathrm{~cm}^{-3} \mathrm{~s}^{-1}$ que tornou-se um ponto de referência 
Figura 3.3 - Taxas de produção de raios gama para uma partícula de massa $500 \mathrm{GeV}$ aniquilando em $b \bar{b}$ (linha sólida) e em $\tau^{+} \tau^{-}$(linha tracejada).

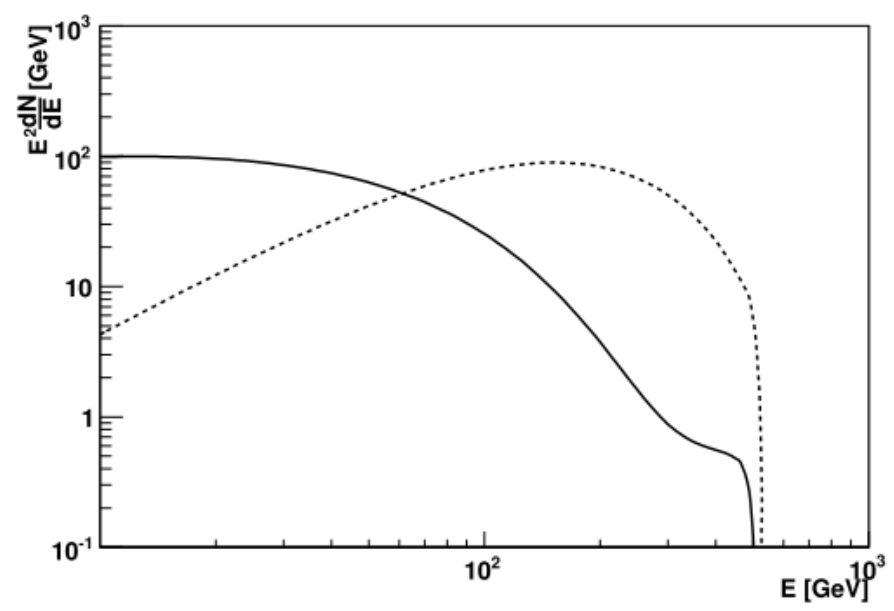

Fonte: WOOD. (50)

inicial para as previsões dos valores de seção de choque.

A outra parte do termo a considerar é o fator $\frac{\mathrm{d} N_{\gamma}}{\mathrm{d} E}$. Este fator representa a taxa de produção de raios gama por aniquilação de partículas de matéria escura. Seu cálculo é dado pela soma das contribuições de todos os canais de aniquilação considerando assim a natureza das partículas secundárias.

Para o neutralino (modelo base do trabalho) os principais canais de aniquilação são os férmions $b \bar{b}, t \bar{t}, \tau^{+} \tau^{-}$, e os bósons $W^{+} W^{-}$e $Z Z$. A contribuição de cada canal de aniquilação tem um peso específico para o perfil total da curva. Por essa razão, encontramos referências nas quais os perfis gerados possuem diferentes porcentagens de diferentes canais de aniquilação. A figura 3.3 mostra dois perfis de taxa de produção de raios gama considerados $100 \%$ em um canal de aniquilação específico: $b \bar{b}$ e $\tau^{+} \tau^{-}$. Os perfis foram gerados usando o pacote PYTHIA Monte Carlo.

O processo de detecção indireta envolve todas as características mencionadas dos termos da expressão de fluxo. Como atualmente só conseguimos estimar um limite superior no fluxo de raios gama quando apontamos para uma fonte específica, o que podemos fazer é limitar as características envolvidas no processo.

Um dado experimental importante para o cálculo é o fluxo integral de raios gama observado de uma fonte específica: $\Phi\left(E_{0}, \Delta \Omega\right)$ onde $E_{0}$ representa a energia de limiar do telescópio. Para obter a expressão de fluxo total, vamos integrar a equação de fluxo diferencial (3.1) em energia. Então, substituindo 3.11 em 3.1 temos que: 


$$
\begin{aligned}
& \frac{\mathrm{d} \Phi}{\mathrm{d} E}(\Delta \Omega)=\frac{<\sigma v>}{2 m_{\chi}^{2}} \frac{\mathrm{d} N_{\gamma}}{\mathrm{d} E} \cdot J(\Delta \Omega) \\
& \int_{E_{0}}^{m_{\chi}} \frac{\mathrm{d} \Phi}{\mathrm{d} E}(\Delta \Omega) \mathrm{d} E=\int_{E_{0}}^{m_{\chi}} \frac{<\sigma v>}{2 m_{\chi}^{2}} \frac{\mathrm{d} N_{\gamma}}{\mathrm{d} E} \cdot J(\Delta \Omega) \mathrm{d} E .
\end{aligned}
$$

Note primeiramente que o limite superior da integração passa a ser $m_{\chi}$ porque, por conservação de energia, a partícula não pode se aniquilar com energias de valores maiores que sua massa. Do lado direito da equação 3.13, observe também que a dependência com energia se restringe apenas ao termo $\frac{\mathrm{d} N_{\gamma}}{\mathrm{d} E}$ (o fator astrofísico $J$ só tem dependência com o ângulo sólido). Dessa forma, temos que:

$$
\Phi\left(E_{0}, \Delta \Omega\right)=J(\Delta \Omega) \cdot \frac{<\sigma v>}{2 m_{\chi}^{2}} \int_{E_{0}}^{m_{\chi}} \frac{\mathrm{d} N_{\gamma}}{\mathrm{d} E} \mathrm{~d} E .
$$

Atualmente, ainda não obtivemos sucesso em determinar um valor de fluxo de raios gama proveniente de matéria escura para uma fonte específica. O que conseguimos é um limite superior no valor de fluxo. Como estamos nos referindo a limites superiores, podemos limitar também a seção de choque de aniquilação restringindo assim as dúvidas com relação às propriedades da partícula. Considerando os limites, temos que:

$$
\Phi\left(E_{0}, \Delta \Omega\right)_{l \text { sup }} \geq J(\Delta \Omega) \cdot \frac{<\sigma v>_{l s u p}}{2 m_{\chi}^{2}} \int_{E_{0}}^{m_{\chi}} \frac{\mathrm{d} N_{\gamma}}{\mathrm{d} E} \mathrm{~d} E
$$

Então,

$$
<\sigma v>_{l s u p} \leq \frac{2 m_{\chi} \Phi\left(E_{0}, \Delta \Omega\right)_{l s u p}}{J_{a n}} \frac{1}{\int_{E_{0}}^{m_{\chi}} \frac{\mathrm{d} N_{\gamma}}{\mathrm{d} E} \mathrm{~d} E}
$$

Utilizaremos a equação 3.16 para realizar os cálculos envolvidos nos dois últimos capítulos desta dissertação.

As perspectivas para melhorar esse tipo de medida e até determinar um valor de fluxo estão atualmente depositadas no futuro CTA (58). Com uma escala de energia de até 4 ordens de magnitude e com melhoras na sensitividade de uma ordem de grandeza, o CTA fará parte de uma nova geração de telescópios para detecção indireta de matéria escura. No próximo capítulo descreveremos algumas das características que o tornam um Observatório de extrema importância para os futuros avanços na determinação e melhoramento dos modelos. 


\section{Cherenkov Telescope Array - CTA}

O Cherenkov Telescope Array é um observatório proposto para estudo de astronomia de raios gama com a colaboração de 29 países. A organização dos componentes para a construção e eventualmente operação do CTA iniciou em 2006 na sua fase de design. Em 2011 a colaboração entrou no período preparatório, no qual os protótipos dos telescópios começaram a ser planejados. Em 2015, esperamos uma revisão geral dos preparativos até agora incluindo a escolha dos países que receberão os telescópios. (59)

O CTA será dividido em dois sítios: um no hemisfério Norte e um no hemisfério Sul. Os países candidatos são Espanha, EUA e México para o Norte e Chile, Namíbia e Argentina para o Sul. Além das características principais para otimizar o funcionamento do CTA, que englobam aspectos geológicos, condições climáticas, entre outros, a condição socioeconômica dos países candidatos a receber a sede do telescópio também é levada em consideração.

O início da fase de construção está previsto para o ano de 2016 e em 2018 espera-se que o CTA já esteja parcialmente operando. (58)

Neste capítulo discutiremos algumas características relacionadas aos telescópios e o que podemos esperar de sua performance.

\subsection{Telescópios Cherenkov}

O Observatório CTA, assim como H.E.S.S. (7), VERITAS (8) e MAGIC (6), será composto por IACTs - Imaging Air Cherenkov Telescopes que são telescópios responsáveis pela detecção de partículas altamente energéticas provenientes de raios gama. Os raios gama interagem com a atmosfera da Terra produzindo uma cascata de partículas que emitem radiação Cherenkov (razão para o nome dos telescópios). Essa cascata tem um máximo no número de partículas que a compõem dependendo essencialmente da energia do raio gama inicial. A energia que chega nos telescópios forma um padrão de imagem que então é analisado para inferir propriedades iniciais da partícula que iniciou a cascata. 
Figura 4.1 - Comparação de sensitividade do Observatório CTA com a de outros observatórios existentes para um tempo de observação de $50 \mathrm{~h}$.

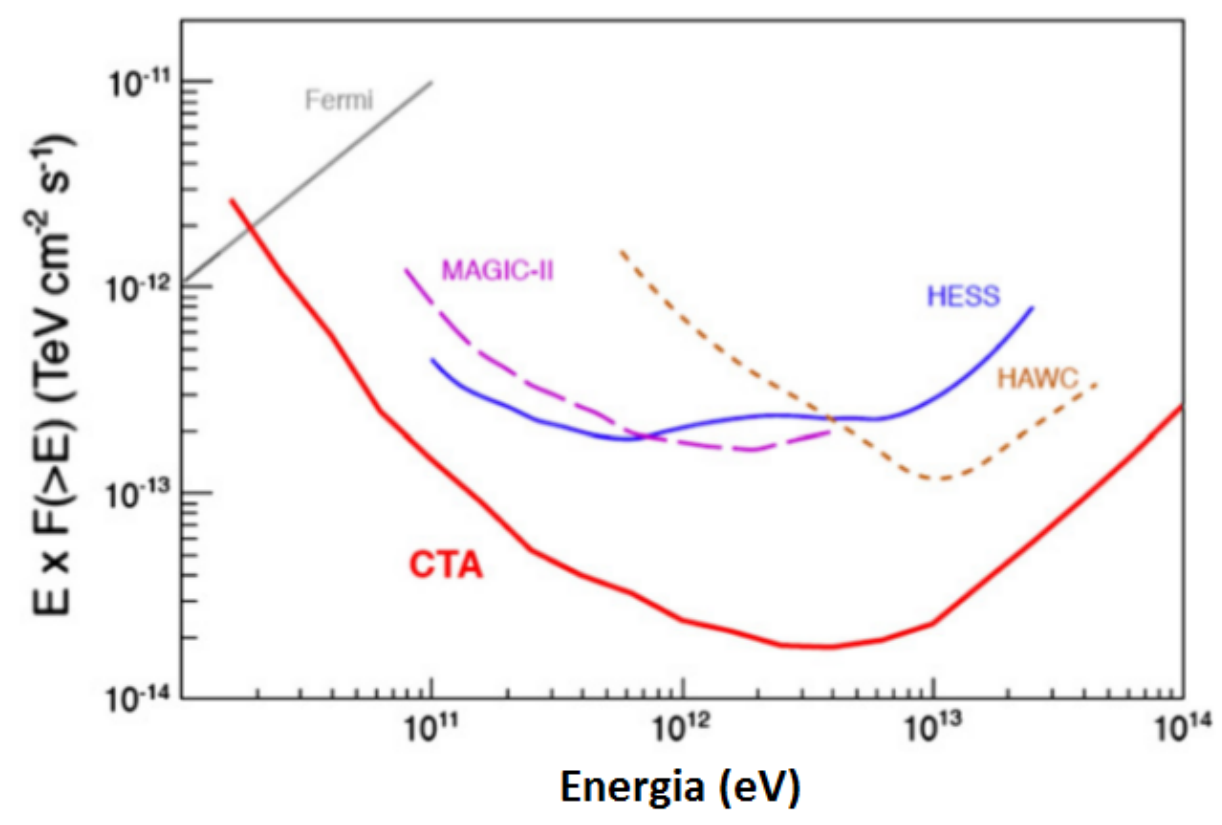

Fonte: Adaptada de ACHARYA. (60)

A radiação Cherenkov é emitida por uma partícula carregada quando esta passa por um meio dielétrico com velocidade maior do que a velocidade da luz nesse meio.

Nesses termos, o CTA faz parte de uma nova geração de telescópios de detecção de partículas altamente energéticas pois pretende aumentar a sensitividade em relação aos IACTS existentes em uma ordem de grandeza e expandir a abrangência em energia de atuação em quatro ordens de grandeza. A figura 4.1 mostra a sensitividade do CTA em comparação à de outros observatórios de raios gama existentes.

\subsection{Sensitividade}

Para atingir os objetivos propostos, o CTA vai contar com três tipos de telescópios: os LSTs - Large Size Telescopes que terão uma abertura de $\sim 24 \mathrm{~m}$ e uma atuação no regime abaixo de $\sim 20 \mathrm{GeV}$, os MSTs - Medium Size Telescopes com uma abertura $\sim 12 \mathrm{~m}$ atuando na escala energética de $\sim 1 \mathrm{TeV}$ e os SSTs - Small Size Telescopes com $\sim 4-7 \mathrm{~m}$ de abertura e otimizado para registrar energias até $\sim 100 \mathrm{TeV}$. (61)

Por essa combinação de telescópios com enfoques diferentes, o CTA operará em um amplo intervalo de energia. O regime de baixas energias vai registrar de alguns $\mathrm{GeV}$ até $\sim 100 \mathrm{GeV}$ 
Figura 4.2 - Exemplos de distribuição dos telescópios $L S T, M S T$ e $S S T$ para os diferentes arranjos B, C e E. O tamanho das circunferências está em escala com o tamanho real dos espelhos de $24 \mathrm{~m}, 12 \mathrm{~m}, 10 \mathrm{~m}$ e $7 \mathrm{~m}$.

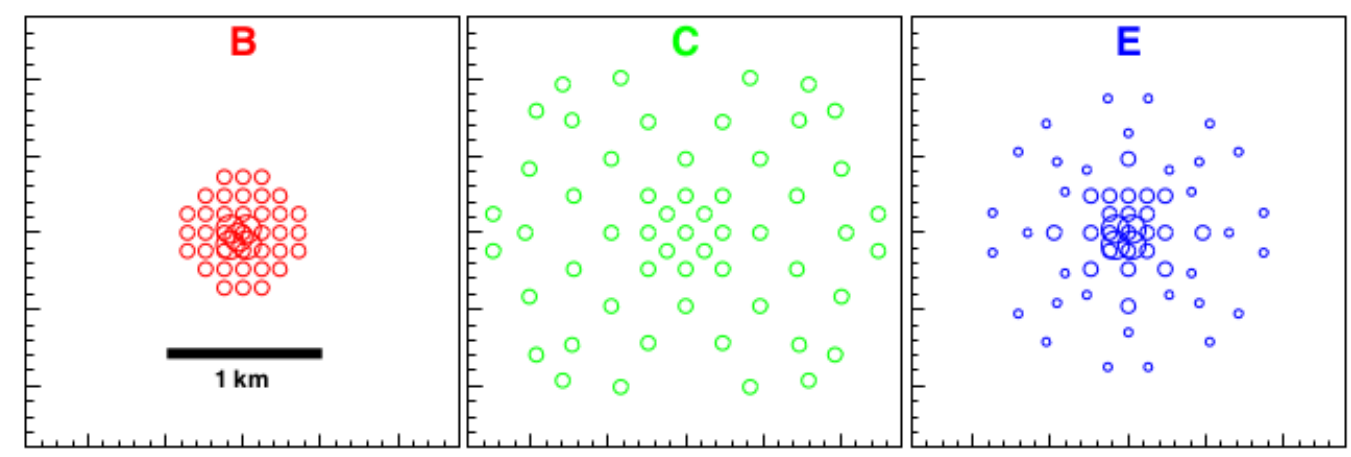

Fonte: Adaptada de ACTIS. (59)

estando principalmente associado aos LSTs para coletar mais fótons Cherenkov provenientes dos raios gama. O regime de médias energias atuando entre $100 \mathrm{GeV}$ e $10 \mathrm{TeV}$ tem o propósito de melhorar observações estereoscópicas aumentando a qualidade das imagens com uma maior densidade de IACTs. O último regime de altas energias se estende até $\approx 100 \mathrm{TeV}$ relacionado com a eficiências dos arranjos de SSTs.

A combinação desses regimes propostos e de diferentes telescópios aliados com o propósito dos programas principais do CTA traz o estudo dos melhores arranjos para uma eficiência máxima do Observatório. Até agora foram elaborados 11 opções de diferentes arranjos para o hemisfério Sul (arranjos A-K) e dois para o hemisfério Norte (arranjos NA e NB) identificados por letras. Atualmente os arranjos estão em fase de teste a fim de optimizar a atuação do CTA. A figura 4.2 mostra alguns exemplos de distribuição dos diferentes telescópios nos arranjos B, C e E.

Note que, a partir da distribuição de telescópios nessas configurações, podemos inferir os regimes preferenciais de cada arranjo. $O$ arranjo $B$ tem uma sensitividade otimizada para altas energias, $\mathrm{O}$ arranjo $\mathrm{C}$ para baixas energias e $\mathrm{o}$ arranjo $\mathrm{E}$ proporciona um equilíbrio entre as demandas de altas e baixas energias. A figura 4.3 mostra o alcance de sensitividade de cada arranjo mencionado.

Até agora, o arranjo $\mathrm{E}$ foi ressaltado como o arranjo que melhor otimiza o balanço entre as energias de atuação e os grupos de telescópios. Dessa forma, há uma preferência para a escolha do arranjo E como base de estudo para características do CTA. Focamos na sensitividade do arranjo E na figura 4.4 É possível notar nesta figura sua a sensitividade total com a contribuição de cada regime de atuação dos três tipos de telescópios.

Para mapear a sensitividade de casos específcos, é preciso utilizar um gerador de chuveiros 
Figura 4.3 - Sensitividades dos arranjos B, C e E. Para cada arranjo, note que há um regime preferencial de atuação conforme aumentamos a energia.

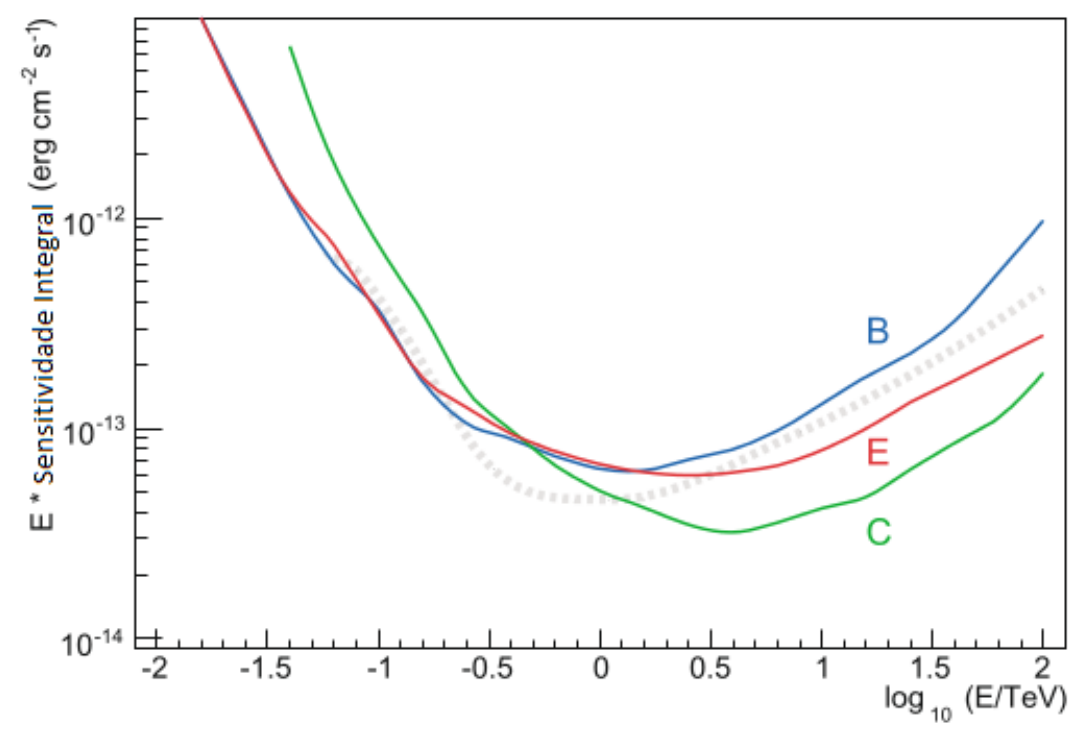

Fonte: Adaptada de ACTIS. (59)

Figura 4.4 - Relação de sensitividade dos tipos de telescópios LSTs, MSTs e SSTs com seus respectivos regimes de atuação para o arranjo $\mathrm{E}(50 \mathrm{~h}, 5 \sigma$, ruído de fundo de $5 \%$ e mínimo de detecção de 10 eventos). A curva preta indica a sensitividade resultante da combinação dos três tipos.

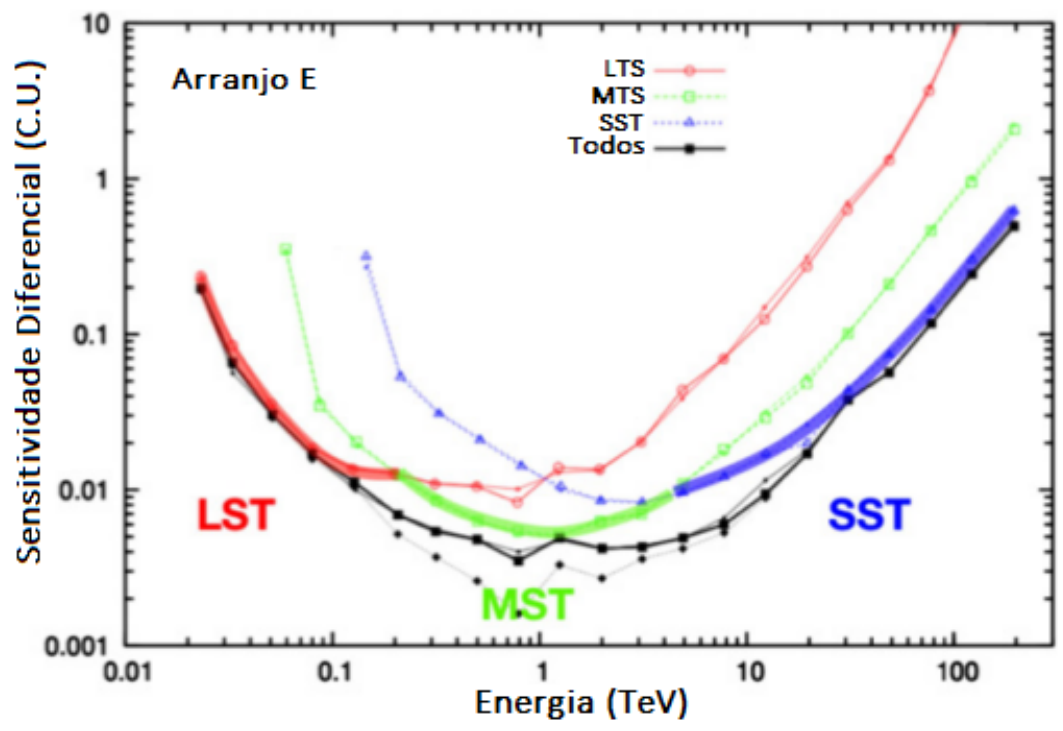

Fonte: Adaptada de ACHARYA. (60) 
Figura 4.5 - Sensitividade do CTA para os arranjos A-K para um sinal com significância $5 \sigma$, com um mínimo de 10 eventos detectados e com um ruído de fundo constituindo $5 \%$ do sinal. O gráfico de cima é simulado para um ângulo zenital de $20^{\circ}$ e o de baixo, para $50^{\circ}$.
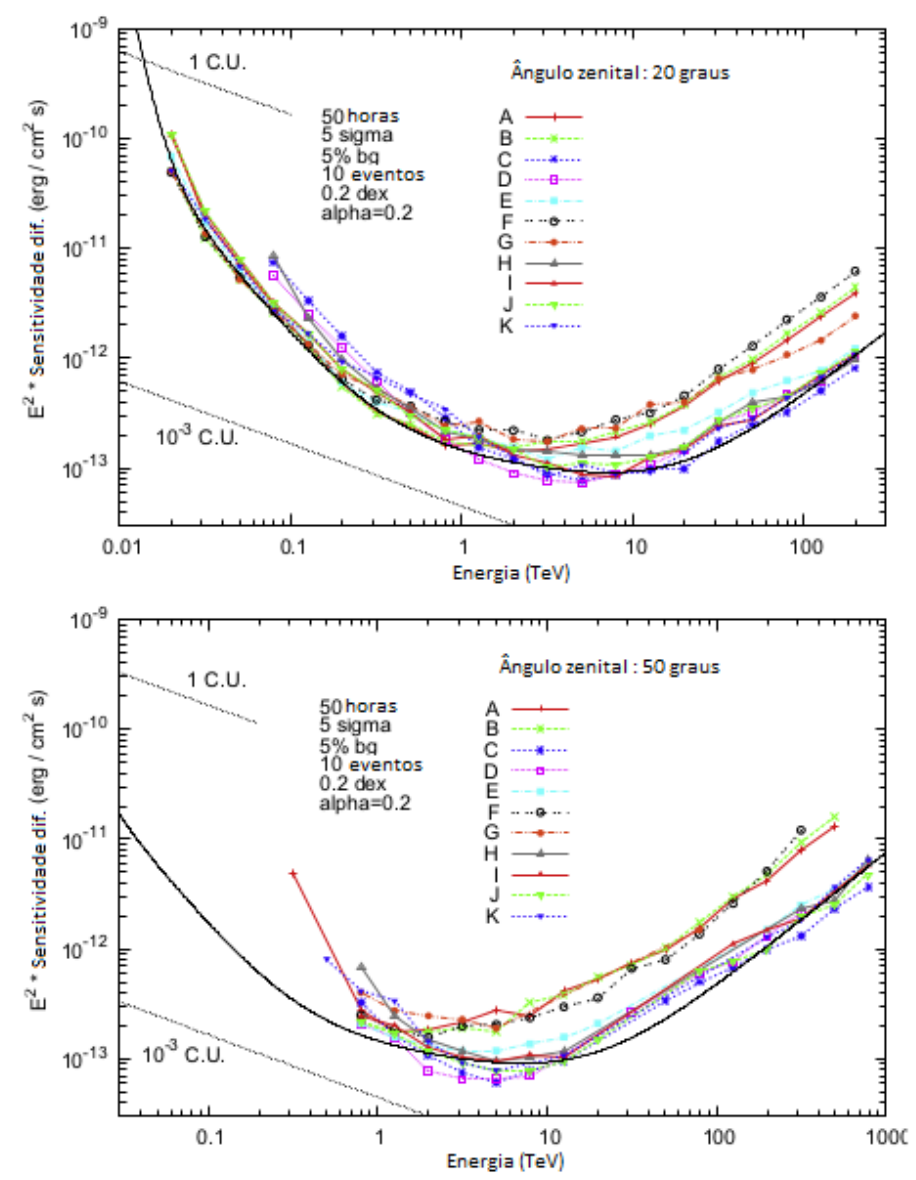

Fonte: Adaptada de BERNLÖHR. (61)

de partículas. Existem vários geradores disponíveis que simulam a cascata de partículas secundárias de forma detalhada. Esses códigos devem considerar as flutuações estatísticas da possível medida e também modelar corretamente as interações hadrônicas do chuveiro. $O$ gerador de chuveiros padrão do CTA é o programa CORSIKA (62).

A figura 4.5 mostra simulações de sensitividade para os arranjos propostos para o hemisfério Sul. Além da análise da distribuição real dos telescópios na aréa do CTA, podemos notar que obtemos uma resposta do instrumento em função do tempo de observação e do ângulo zenital em que a fonte é visível.

Podemos dividir a influência do tempo de observação na sensitividade em duas partes: o comportamento quase linear com $t$ na região dos eventos de raios gama e um comportamento aproximado de $t^{(1 / 2)}$ nas regiões de menores energias. A figura 4.6 mostra a sensitividade do arranjo E para diferentes tempos de observação $(T=0.5 \mathrm{~h}, T=5 \mathrm{~h}$ e $T=50 \mathrm{~h})$. As curvas vermelha e azul correspondem a rejeição dos chuveiros iniciados por outras partículas. 
Figura 4.6 - Sensitividade do arranjo E para três tempos de observação diferentes com um sinal de significância $5 \sigma$, possuindo um mínimo de 10 eventos detectados e uma contribuição de $5 \%$ de ruído de fundo. A curva vermelha representa a rejeição hadrônica simulada a partir de um método estatístico chamado Random Forests e a azul, a partir de uma análise utilizando TMVA Toolkit for Multivariate Data Analysis.

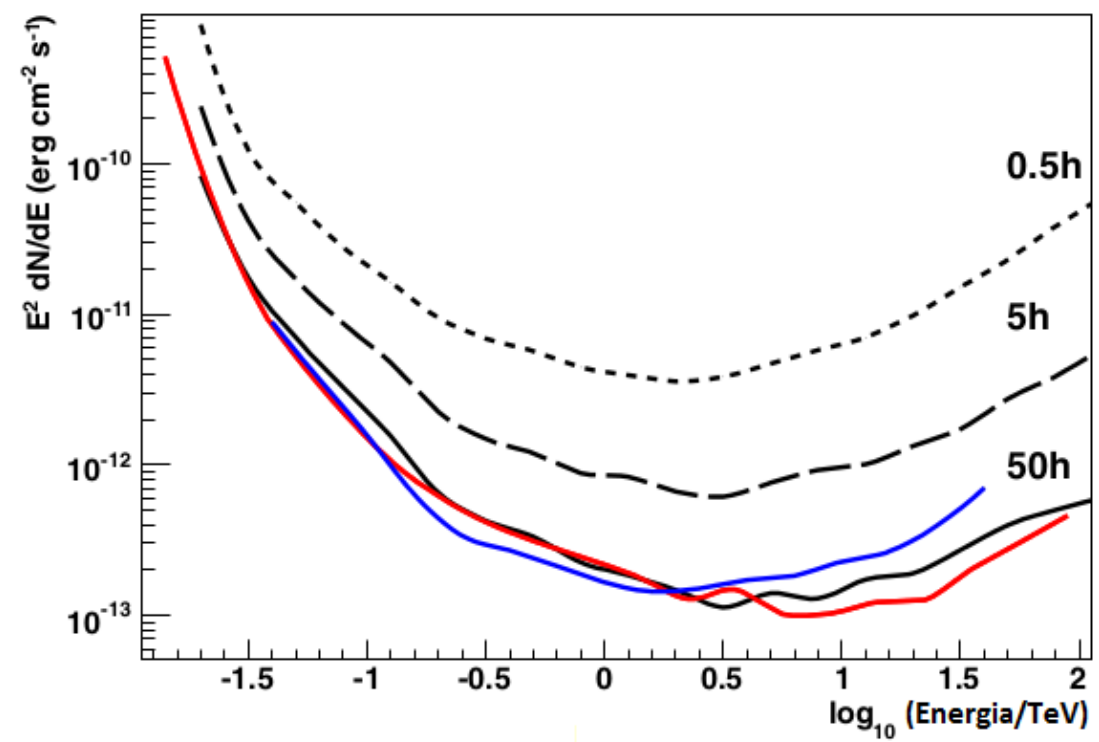

Fonte: Adaptada de ACTIS. (59)

\subsection{Estudo da matéria escura pelo CTA}

Um dos objetivos principais do CTA é o entendimento sobre a natureza física além do Modelo Padrão. Nesse contexto, há grande expectativa pela comunidade científica com relação ao estudo de matéria escura pelas seguintes razões:

- No intervalo de energias alcançadas pelo CTA, para baixas energias, será possível sobrepor regiões do experimento FERMI-LAT. Isso possibilitará o estudo de partículas de matéria escura de menor massa;

- Com o aumento em uma ordem de grandeza na sensitividade comparada com os instrumentos atuais, a propabilidade de detecção também aumenta;

- O aumento no campo de visão do telescópio e a sensitividade mais homogênea proporcionam buscas mais efetivas para fontes extensas;

- A resolução de energia do CTA permite uma maior sensitividade na análise dos espectros de energias registrados. 
Figura 4.7 - Curvas de sensitividade do CTA sobre seção de choque de aniquilação por massa da partícula de matéria escura com significância $5 \sigma$ e com tempo de observação $T=100 \mathrm{~h}$ para o arranjo $\mathrm{E}$.

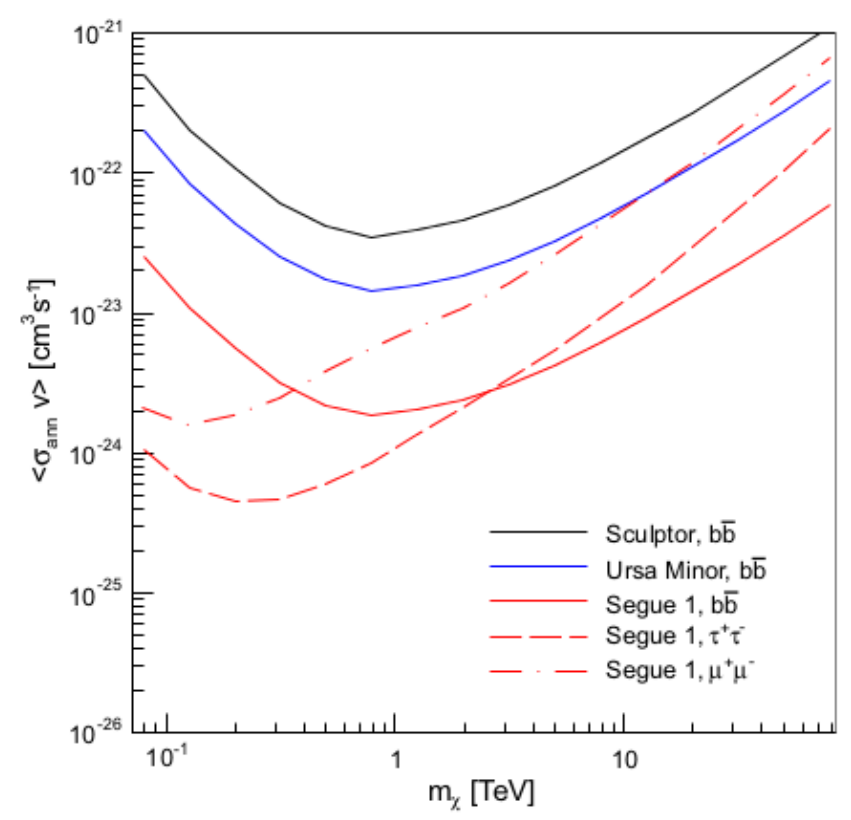

Fonte: DORO. (22)

Para optimizar a performance do CTA com relação a diferentes fontes e configurações, previsões são realizadas a fim de definir algumas características.

A figura 4.7 mostra as previsões dos limites superiores de seção de choque para as galáxias Sculptor, Ursa Minor e Segue 1 apresentados na referência (22). Para Segue 1, foram apresentadas curvas para os canais de aniquilação $b \bar{b}, \tau^{+} \tau^{-}$e $\mu^{+} \mu^{-}$e para Sculptor e Ursa Minor apenas para o canal $b \bar{b}$.

Utilizaremos esses resultados no capítulo 6 para estudar o efeito do tempo de observação sobre os limites de seção de choque calculados por fonte.

Para aglomerados de galáxias, o estudo de matéria escura se torna mais complexo por possuir um ruído de fundo alto. Para distinguir emissão dos raios cósmicos da emissão de matéria escura, a optimização da região de interesse na imagem do CTA é aplicada. A figura 4.8 mostra o fluxo de raios gama do aglomerado Fornax optimizado para separar a contribuição de raios cósmicos e de matéria escura. É possível notar que a partir de $\theta=0.4^{\circ}$ o sinal é dominado pela emissão de raios gama proveniente da aniquilação de matéria escura. Ou seja, a princípio a contribuição de matéria escura poderia ser isolada escolhendo uma região de interesse $\operatorname{com} \theta_{\min }=0.4^{\circ}$. 
Figura 4.8 - Curva de fluxo de raios gama do aglomerado de galáxias Fornax. A curva vermelha representa a contribuição de raios cósmicos para o sinal, a curva azul, a contribuição de matéria escura e em preto, a soma das duas.

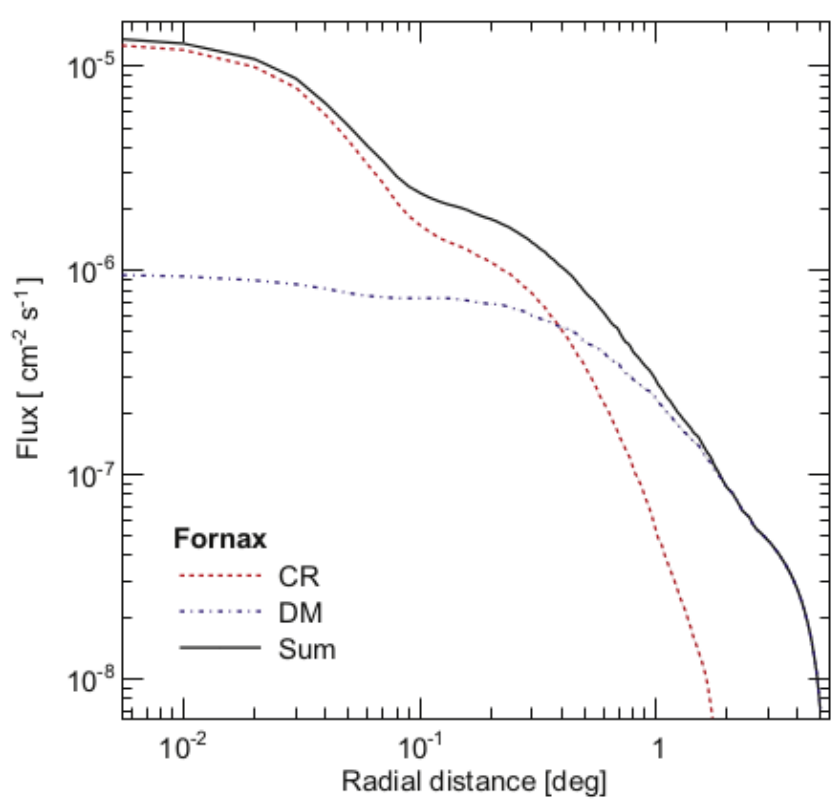

Fonte: DORO. (22)

Podemos citar também a detecção de matéria escura no Centro Galáctico. Como o ruído de fundo de medidas de fluxo de raios gama do Centro Galáctico também é muito alto, o método para isolar a contribuição de matéria escura para o sinal é realizando medidas em uma região que contenha apenas contribuição de ruído de fundo (OFF region), de uma região com contribuições de matéria escura e de ruído de fundo ( $O N$ region) e então subtraí-las. A figura 4.9 mostra a sensitividade do CTA para seção de choque de aniquilação para os canais de aniquilação $b \bar{b}, \tau^{+} \tau^{-}$e $\mu^{+} \mu^{-}$. As curvas são calculadas para os arranjos $\mathrm{E}$ e B. O método de subtração de regiões utilizado é o Ring Method. (22) 
Figura 4.9 - Sensitividade do CTA para a seção de choque de aniquilação da partícula de matéria escura no Centro Galáctico para os arranjos E e B. As curvas sólidas correspondem ao canal de aniquilação $b \bar{b}$, as pontilhadas ao canal $\mu^{+} \mu^{-}$e as tracejadas ao canal $\tau^{+} \tau^{-}$.

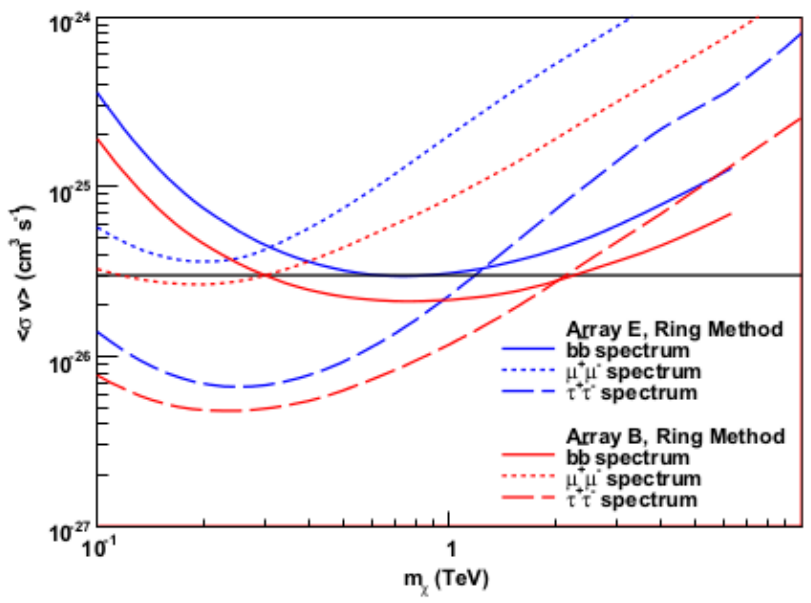

Fonte: DORO. (22) 


\section{Cálculo de limites na seção de choque de aniquilição}

Como já mencionamos todos os elementos principais para a detecção indireta de matéria escura, neste capítulo explicaremos o processo de obtenção dos primeiros resultados. Unimos as informações dos primeiros capítulos para extrair resultados conclusivos sobre o cálculo da seção de choque de aniquilação da partícula de matéria escura.

Para essa etapa, coletamos informações e dados de duas referências (MAGIC (6) e VERITAS (8)), implementamos um código que suprisse as necessidades computacionais e checamos a concordância dos resultados. Nas próximas seções detalharemos o processo.

Para as duas referências, as fontes envolvidas no cálculo são galáxias anãs esferoidais que podemos classificar entre Ultra-fracas (Ultra-faint) e Clássicas. As Ultra-faint são galáxias com baixa luminosidade em torno de $L \leq 10^{5} L_{\odot}$ e caracterizadas por terem apenas dezenas de estrelas, resultando em uma baixa população estelar. Tipicamente possuem um raio de meia luz (half-light: raio do centro da galáxia até metade de seu comprimento luminoso) entre $30-$ $100 \mathrm{kpc}$. Algumas galáxias ultra-fraca ainda não estão muito bem estabelecidas porque essa classe possui propriedades similares à de aglomerados globulares dificultando sua identificação. Já as clássicas possuem centenas de estrelas com luminosidade entre $10^{5}-10^{7} L_{\odot}$. São mais extensas e mais luminosas do que as galácias ultra-fracas, e estão a distâncias de 70 a $250 \mathrm{kpc}$.

As galáxias envolvidas nesses próximos cálculos são Draco (Clássica) e Segue 1 (Ultrafaint). As duas estão situadas fora do plano galáctico com razões massa-luminosidade de $\approx 3400 \frac{M_{\odot}}{L_{\odot}}$ para Segue 1 e de $\approx 400 \frac{M_{\odot}}{L_{\odot}}$ para Draco. A tabela 5.1 mostra algumas propriedades das duas galáxias.

Cálculamos os limites superiores na seção de choque de aniquilação a partir de dados das referências (28) e (21), respectivamente. As análises apresentadas nas referências limita o possível valor de seção de choque de aniquilação dado um modelo de partícula e uma fonte específica. Como mencionado anteriormente, estudamos a aniquilação de neutralinos em galáxias anãs esferoidais. O objetivo, nesta seção, é validar nossa metodologia e implementação para o cálculo do limite superior da seção de choque. 
Tabela 5.1 - Propriedades das galáxias Draco e Segue 1 onde $d$ é a distância da galáxia à Terra, $M_{v}$ é a magnitude absoluta, $L$ corresponde à luminosidade em unidade de luminosidade do sol, $r_{h}$ é o raio half-light (distância do centro da galáxia até metade de seu raio luminoso) e $\frac{M}{L}$, a razão massa-luminosidade de cada uma.

\begin{tabular}{lccccc}
\hline & $d[k p c]$ & $M_{v}$ & $L\left[10^{6} L_{\odot}\right]$ & $r_{h}[p c]$ & $\frac{M}{L}\left[\frac{M_{\odot}}{L_{\odot}}\right]$ \\
\hline Draco & 80 & -9.4 & 0.26 & 181 & 400 \\
Segue 1 & 23 & -1.5 & $3.4 \cdot 10^{-4}$ & 29 & 3400 \\
\hline \multicolumn{5}{c}{ Fonte: Adaptado de ALEKSIC. (28) }
\end{tabular}

\subsection{Estudo referente à análise do MAGIC}

Para reproduzirmos os resultados do MAGIC, extraímos os dados necessários para trabalharmos com a equação 3.16 Estudamos a galáxia Segue I e os canais de aniquilação do neutralino $b \bar{b}, \tau^{+} \tau^{-}$e $W^{+} W^{-}$. A comparação dos valores obtidos com os valores da referência (28) foi apenas realizada para o canal $b \bar{b}$. Não foi possível comparar resultados referentes ao outros canais de aniquilação porque não estavam disponíveis. Efetuamos os cálculos mesmo assim a fim de estabalecermos uma base de entendimento independente de uma escolha específica como o canal de aniquilação do neutralino.

\subsubsection{Fluxo de raios gama e fator astrofísico $J$}

Começaremos a apresentar os dados utilizados com os valores de fluxo de raios gama. A obtenção desses valores pelos telescópios Cherenkov vem da análise do espectro de energia das imagens registradas do chuveiro de partículas. Depois da análise, é possível definir uma função para descrever o comportamento do fluxo de raios gama por energia da fonte. Tipicamente são utilizadas leis de potências para essa descrição. Na referência (28), são apresentados seis diferentes expoentes da lei de potências: $\Gamma=-1.0,-1.5,-1.8,-2.0,-2.2$ e -2.4 . As figuras 5.1 e 5.2 mostram, respectivamente, o gráfico dos limites superiores do fluxo diferencial e do fluxo integral de raios gama correspondentes aos diferentes expoentes estimados.

Para reproduzirmos os resultados obtidos com os dados do MAGIC, selecionamos o fluxo integral com o maior valor de limiar de energia de $\approx 60 \mathrm{GeV}$. O maior valor possível de fluxo é vantajoso para o objetivo do trabalho porque não restringe o resultado a observações especiais ou a lei de potências mais restritivas, o que resulta em uma maior validade para o valor obtido no final dos cálculos. Porém, essa escolha implica em selecionarmos um só 
Figura 5.1 - Limites superiores do fluxo diferencial observados pelo MAGIC com diferentes expoentes da lei de potências estimada. A linha sólida representa o fluxo diferencial da Nebulosa de Carangueijo, a reta com longos traços representa $10 \%$ desse fluxo e a com traços menores $1 \%$.

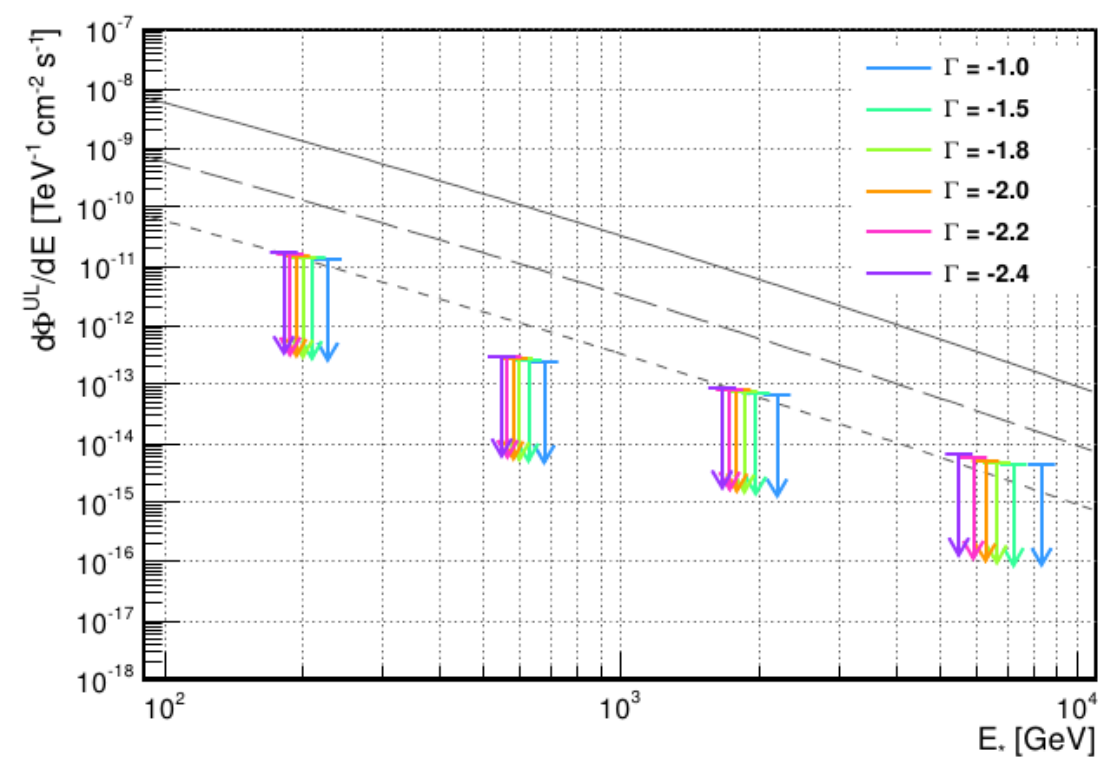

Fonte: ALEKSIC. (28)

Figura 5.2 - Limites superiores do fluxo integral considerando os diferentes expoentes da lei de potências. As linhas tracejadas representam as funções aproximadas para os valores de limite superior de fluxo.

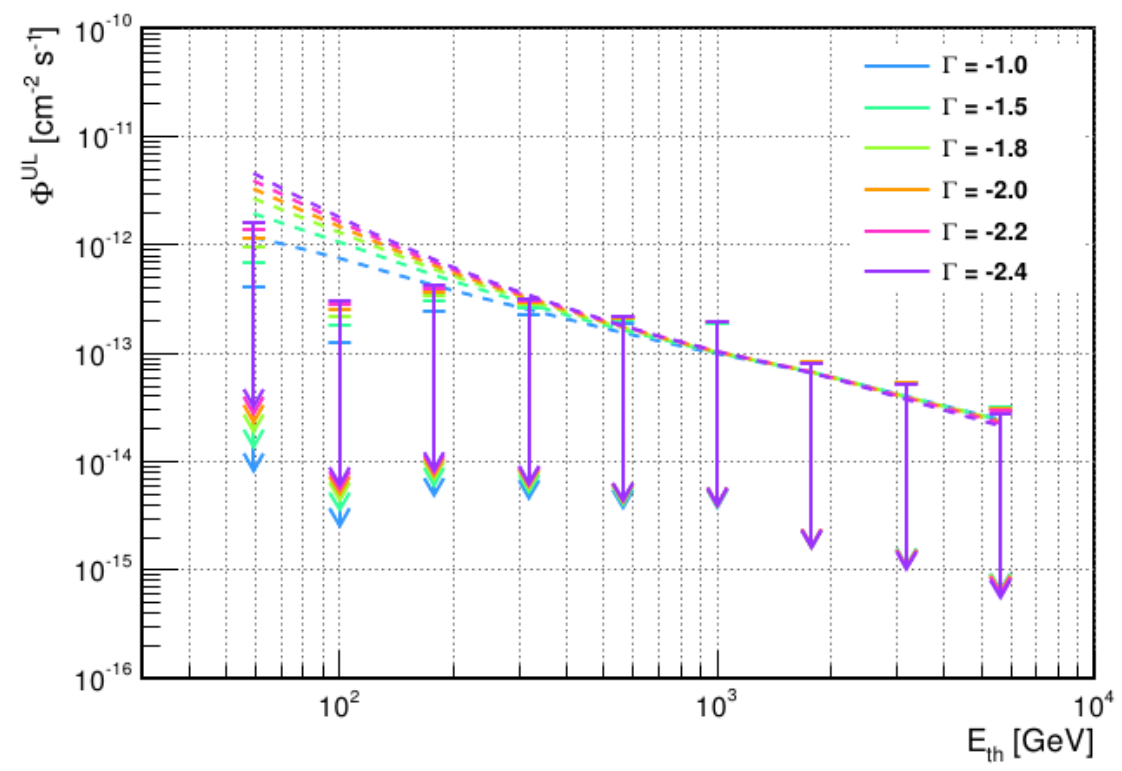

Fonte: ALEKSIC. (28) 
expoente para a lei de potências da referência (28). Portanto, o valor de fluxo que adotamos foi $\Phi_{l \text { sup }}=1.62 \cdot 10^{-12} \mathrm{~cm}^{-2} \mathrm{~s}^{-1}$.

Outro aspecto importante do cálculo é o valor do fator astrofísico $J$. Na referência (28), $J$ foi modelado a partir do perfil Einasto (27) onde o raio de escala $a=0.15 \mathrm{kpc}$ e $\alpha=0.3$. Um estudo foi realizado para otimizar o ângulo $\theta$ na integração do ângulo sólido $\Delta \Omega$ como mencionado na seção 3.1.1 para fontes extensas. Após as considerações de perfil e ângulo da análise de (28), chegamos ao valor de $J_{a n}=1.05 \cdot 10^{19} \mathrm{GeV}^{2} \mathrm{~cm}^{-5} \mathrm{sr}$. Esse valor é o que consideramos nos cálculos.

\subsubsection{Taxa de produção de raios gama por aniquilação de partícu- las}

Para obtermos a função que descreve o perfil da taxa de produção de raios gama $\frac{\mathrm{d} N_{\gamma}}{\mathrm{d} E}$ utilizado para derivar os limites na referência, precisamos extrair a informação dos gráficos que representam essa taxa.

Os perfis de produção de raios gama da referência (28) consideram a resposta do telescópio MAGIC sob a simulação do gerador de eventos. Em outras palavras, na análise das taxas é levada em conta a convolução da energia medida com a energia simulada pelo gerador de eventos resultando no valor de energia que realmente participa do resultado final. Portanto consideramos apenas a curva da função convoluída sobre as energias. Na figura 5.3 estão os perfis para dois canais distintos $b \bar{b}$ e $\tau^{+} \tau^{-}$.

\subsubsection{Implementação}

Até agora, possuímos os valores de fluxo integral, do fator astrofísico $J_{a n}$ e os gráficos que descrevem as curvas da taxa de produção de raios gama por interação. Das equações 3.15 e 3.16 podemos ver que, para começarmos a estimar valores de limite superior na seção de choque, precisamos integrar o perfil $\frac{\mathrm{d} N_{\gamma}}{\mathrm{d} E}$ em energia.

Para isso utilizamos o software Digitizelt para extrair pontos correspondentes à curva do perfil de produção de raios gama por aniquilação. Aproximamos uma função e integramos em energia com a plataforma Root. Na integração, o limite inferior corresponde ao valor de energia de limiar e o limite superior assume um valor escolhido pelo usuário de forma que seja 
Figura 5.3 - Perfis da taxa de produção de raios gama $\frac{\mathrm{d} N_{\gamma}}{\mathrm{d} E}$ para massas de $100 \mathrm{GeV}, 1000 \mathrm{GeV}$ e $10000 \mathrm{GeV}$ assumindo o canal de aniquilação $b \bar{b}$ (em cima) e $\tau^{+} \tau^{-}$(embaixo). As curvas tracejadas representam as funções resultantes da convolução sobre as energias do modelo gerado e medidas.
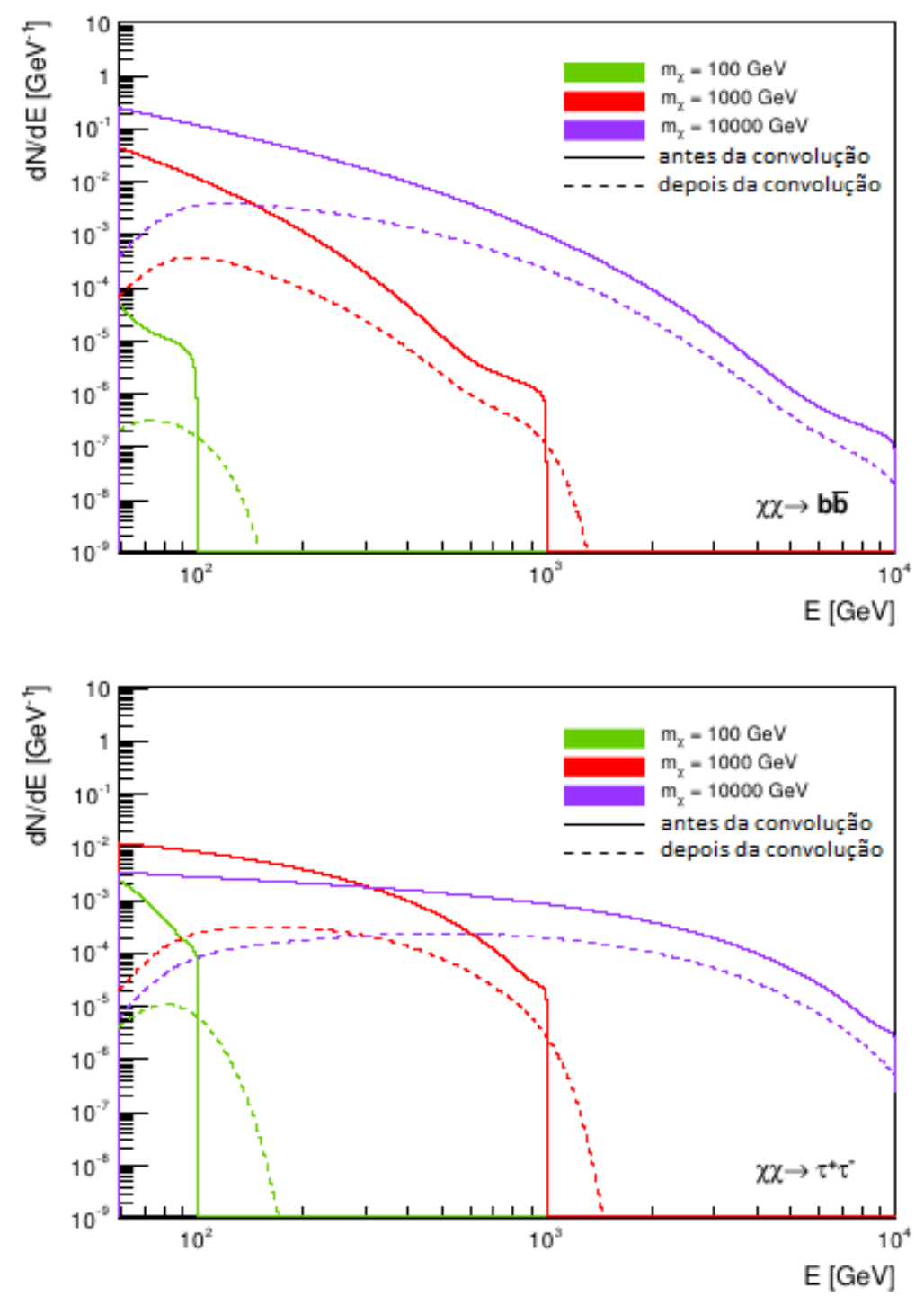

Fonte: ALEKSIC. (28) 
Figura 5.4 - Perfil de produção de raios gama gerado para o canal de aniquilação $b \bar{b}$ com massa $m_{\chi}=1000 \mathrm{GeV}$. Os pontos extraídos são referentes ao gráfico contido na referência (28). A curva vermelha indica a função que caracteriza os pontos.

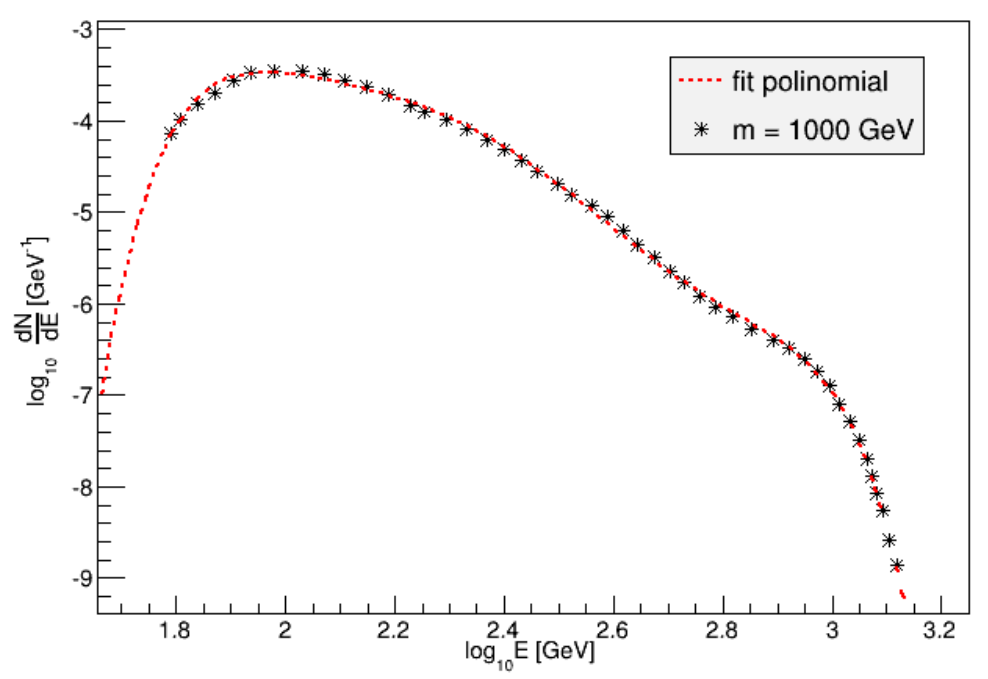

Fonte: Elaborada pela autora.

maior do que a massa da partícula de matéria escura $m_{\chi}$ : isso acontece para que o limite não funcione como um corte no gráfico, ou seja, consideramos a suavização da curva quando esta começa a tender a zero. A figura 5.4 apresenta o gráfico gerado com os pontos extraídos e a função aproximada para o canal de aniquilação do neutralino $b \bar{b}$ de massa $1000 \mathrm{GeV}$.

Na referência (28), a análise dos dados do MAGIC contém uma otimização dos tempos de observação e das leis de potências que expressam os fluxos. Como o resultado final na referência (28) considera esses procedimentos, o que podemos comparar são os valores de um só tempo de observação e os obtidos anteriormente ao processo de otimização das leis de potências. Nessas condições, há somente disponibilidade dos resultados de limite superior de seção de choque para o canal de aniquilação $b \bar{b}$. Realizamos o mesmo procedimento para outros canais, mas não estão disponíveis informações necessárias para compararmos os valores de seção de choque.

Com a integral calculada, substituímos o valor na equação 3.16 para estimarmos o limite na seção de choque de aniquilação. Na tabela 5.2 estão os valores obtidos com a implementação e os valores da referência. A tabela 5.3 indica os valores obtidos para os canais de aniquilação $\tau^{+} \tau^{-}$e $W^{+} W^{-}$. Para obter os valores de erro, propagamos o erro da aproximação da função e do valor de $J$. Predominantemente, o erro do fator $J\left(\approx 10^{-1}\right)$ é muito maior do que o erro cometido na aproximação da função $\left(\approx 10^{-4}\right)$. De modo geral, as comparações dos limites de seção de choque para o canal de aniquilação $b \bar{b}$ com os resultados da referência (28) não proporcionaram valores satisfatórios. Apesar de relativa proximidade, as diferenças 
encontradas não foram possíveis de explicação. Conferimos os passos de cada cálculo de seção de choque de aniquilação com a autora da referência (28) que julgou os resultados como satisfatórios.

Tabela 5.2 - Valores obtidos com a implementação do algoritmo e com os dados de (28) para o canal de aniquilação $b \bar{b}$ (à esquerda) comparados com os valores originais da referência.

\begin{tabular}{ccc}
\hline massa $[\mathrm{TeV}]$ & $\left\langle\sigma v>_{\text {calculado }}\left[\mathrm{cm}^{3} \mathrm{~s}^{-1}\right]\right.$ & $\left\langle\sigma v>_{\text {referência }}\left[\mathrm{cm}^{3} \mathrm{~s}^{-1}\right]\right.$ \\
\hline 0.1 & $(1.9 \pm 0.2) \cdot 10^{-21}$ & $1.1 \cdot 10^{-21}$ \\
1 & $(5.6 \pm 0.9) \cdot 10^{-23}$ & $4.0 \cdot 10^{-23}$ \\
10 & $(1.5 \pm 0.2) \cdot 10^{-22}$ & $6.0 \cdot 10^{-23}$ \\
\hline
\end{tabular}

Fonte: Elaborado pela autora.

Tabela 5.3 - Valores do limite superior da seção de choque calculados pela implementação dos canais $\tau^{+} \tau^{-}$ e $W^{+} W^{-}$. Erros calculados por propagação de erro.

\begin{tabular}{ccc}
\hline massa $[\mathrm{TeV}]$ & $\left\langle\sigma v>_{\tau^{+} \tau^{-}}\left[\mathrm{cm}^{3} \mathrm{~s}^{-1}\right]\right.$ & $\left\langle\sigma v>_{W^{+} W^{-}}\left[\mathrm{cm}^{3} \mathrm{~s}^{-1}\right]\right.$ \\
\hline 0.1 & $(1.1 \pm 0.9) \cdot 10^{-23}$ & $(3.1 \pm 0.3) \cdot 10^{-21}$ \\
1 & $(2.9 \pm 0.4) \cdot 10^{-23}$ & $(8.9 \pm 0.9) \cdot 10^{-23}$ \\
10 & $(7.7 \pm 0.8) \cdot 10^{-22}$ & $(3.7 \pm 0.4) \cdot 10^{-22}$ \\
\hline
\end{tabular}

Fonte: Elaborado pela autora.

\subsection{Estudo referente a análise do VERITAS}

Nesta seção, o procedimento para reprodução dos resultados de limite superior de seção de choque é análogo ao descrito na seção anterior. Para a análise referente ao experimento do VERITAS, extraímos os dados da referência. (21)

Estudamos duas galáxias anãs esferoidais presentes na referência citada, Draco e Segue I. Diferentemente do realizado na seção anterior, na qual escolhemos um canal de aniquilação específico, neste procedimento trabalhamos com a combinação dos canais $b \bar{b}$ e $\tau^{+} \tau^{-}$.

\subsubsection{Fluxo de raios gama e fator astrofísico J}

A obtenção dos fluxos de raios gama de uma fonte pelo VERITAS funciona no mesmo padrão descrito na seção 5.1.1 A diferença é o método utilizado por cada telescópio para a 
optimização das imagens e resultados correspondentes. Na referência (21), são considerados dois importantes cortes para os eventos que resultam nos valores de fluxo: corte padrão (standard) e curte suave (soft cut). O corte padrão considera uma lei de potências com expoente $\Gamma=2.5$ e o corte suave, $\Gamma=3.5$, sendo sua principal diferença o alcance da energia de limiar no qual o corte suave proporciona valores de $100-200 \mathrm{GeV}$ menores que o corte padrão.

A tabela 5.4 apresenta os valores de fluxo diferencial e fluxo integral para as fontes estudadas a partir da análise com o corte suave. Como apresentado, utilizaremos $\Phi=$ $9.5 \cdot 10^{-12} \mathrm{~cm}^{-2} \mathrm{~s}^{-1}$ e $\Phi=2.2 \cdot 10^{-12} \mathrm{~cm}^{-2} \mathrm{~s}^{-1}$ para os valores de fluxo superior de raios gama para Draco e Segue I, respectivamente.

Tabela 5.4 - Valores de limite de fluxo diferencial e integral respectivamente da referência (21). Para o fluxo integral, a energia de limiar foi de $100 \mathrm{GeV}$.

\begin{tabular}{ccc}
\hline Fonte & $E^{2} \frac{\mathrm{d} \Phi}{\mathrm{d} E}\left[\mathrm{erg} \mathrm{m}^{-2} \mathrm{~s}^{-1}\right]$ & $\Phi_{\text {lsup }}\left[\mathrm{m}^{-2} \mathrm{~s}^{-1}\right]$ \\
\hline Draco & $1.3 \cdot 10^{-8}$ & $9.5 \cdot 10^{-8}$ \\
Segue 1 & $4.9 \cdot 10^{-9}$ & $2.2 \cdot 10^{-8}$ \\
\hline
\end{tabular}

Fonte: Adaptado de WOOD. (21)

O valor do fator astrofísico $J$ foi avaliado para cada uma das fontes pelos perfis de densidade de matéria escura NFW (20) e Hernquist (26). A referência (21) adota uma convenção para expressar $J$ adimensionalmente da seguinte forma:

$$
\tilde{J}=\frac{J}{\rho_{c}^{2} R_{H}},
$$

onde $\rho_{c}^{2}$ representa a densidade crítica do Universo e $R_{H}$ o raio de Hubble. A densidade crítica $\rho_{c}^{2}$ é uma constante que, quando comparada com a densidade atual, determina a geometria do Universo. Caso observássemos a relação,

$$
\Omega=\frac{\rho}{\rho_{c}}=1
$$

onde $\Omega$ é o parâmetro de densidade e $\rho$ a densidade atual do Universo, estaríamos afirmando que o Universo é plano. O raio de Hubble $R_{H}$, em termos breves, representa o raio do Universo observável com relação ao observador. Como $J$ também está relacionado com densidade e raio da fonte em questão, essa convenção proporciona uma visão comparativa com as constantes do Universo.

Dessa forma, a relação se torna: 


$$
\tilde{J}=\frac{J}{\rho_{c}^{2} R_{H}}=\frac{J}{3.832 \cdot 10^{17} \mathrm{GeV}^{2} \mathrm{~cm}^{-5}}
$$

Nos cálculos produzidos a partir dos dados dessa referência, estamos considerando apenas o perfil NFW. A tabela 5.5 mostra os valores de $\tilde{J}$ para as fontes em questão.

Tabela 5.5 - Valores do fator $\tilde{J}$ para as galáxias Draco e Segue I a partir referência (21) calculados a partir dos perfis de densidade NFW (20) e Hernquist (26) respectivamente.

\begin{tabular}{ccc}
\hline Fonte & $\log _{10}\left(\tilde{J}_{N F W}\right)$ & $\log _{10}\left(\tilde{J}_{H}\right)$ \\
\hline Draco & $0.09 \pm 0.19$ & $0.06 \pm 0.19$ \\
Segue 1 & $1.07 \pm 1.80$ & $1.12 \pm 0.80$ \\
\hline
\end{tabular}

Fonte: Adaptado de WOOD. (21)

Com as conversões do logaritmo e considerando a convenção adotada para $J$ adimensional, os valores do fator astrofísico $J$ para Draco e Segue 1 correspondem, respectivamente a $4.71 \cdot 10^{17} \mathrm{GeV}^{2} \mathrm{~cm}^{-5} \mathrm{sr}$ e $4.50 \cdot 10^{18} \mathrm{GeV}^{2} \mathrm{~cm}^{-5} \mathrm{sr}$.

\subsubsection{Taxa de produção de raios gama por aniquilação de partícu- las}

Os perfis de taxa de produção de raios gama da referência (21) foram gerados com o pacote DarkSUSY (63). A apresentação dos dados do perfil é semelhante à da referência (28) pelo MAGIC. Na figura 5.5 podemos ver os perfis do neutralino para massas diferentes considerando a aniquilação nas contribuições dos canais $90 \% b \bar{b}$ e $10 \% \tau^{+} \tau^{-}$.

Uma vez com os perfis de produção de raios gama por aniquilação e os fatores astrofísicos $J$, realizamos o mesmo procedimento de extração dos pontos com o software Digitizelt (64) e implementamos um código similar ao da seção anterior para a aproximação e eventual cálculo da integral com a plataforma Root (65). A figura 5.6 apresenta o gráfico gerado com a implementação para uma partícula de massa $m_{\chi}=1000 \mathrm{GeV}$. Os pontos representam a curva extraída da referência (21) e a curva, a função aproximada.

Realizamos o mesmo procedimento para as duas fontes Draco e Segue 1 a fim de limitar a seção de choque de aniquilação. Para fins comparativos, a figura 5.7 mostra os limites superiores da seção de choque dependente da massa elaborados na referência (21). As tabelas abaixo 5.6 e 5.7 contem os resultados para Draco e Segue 1, respectivamente. 
Figura 5.5 - Perfis de produção de raios gama $\left(\frac{\mathrm{d} N_{\gamma}}{\mathrm{d} E}\right)$ considerando massas de $316 \mathrm{GeV}$ (curva preta), $1 \mathrm{TeV}$ (curva vermelha), $3.16 \mathrm{TeV}$ (curva verde) e $10 \mathrm{TeV}$ (curva azul). Todas as curvas são provenientes da aniquilação de neutralinos pelas contribuições $90 \% b \bar{b}$ e $10 \% \tau^{+} \tau^{-}$.

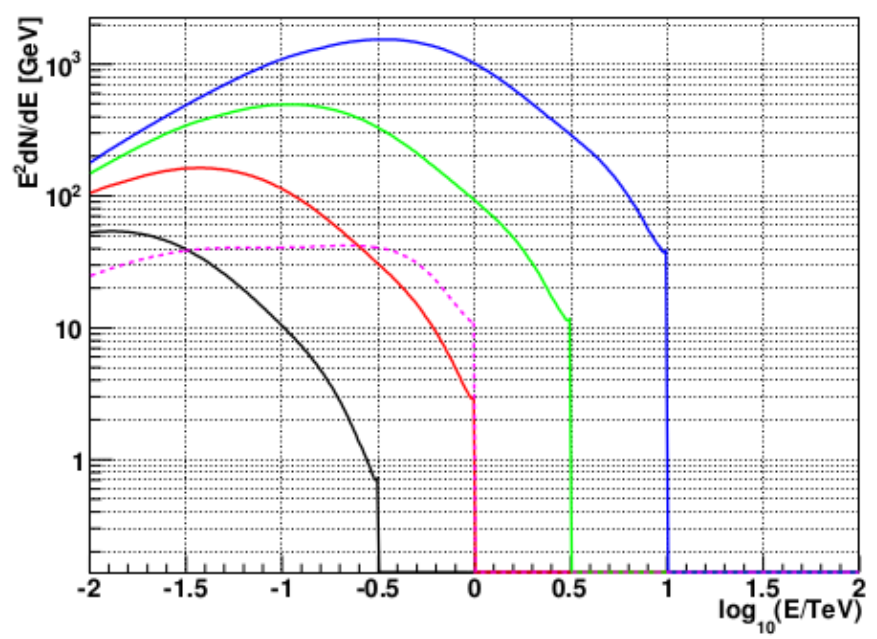

Fonte: Adaptada de WOOD. (21)

Figura 5.6 - Perfil de produção de raios gama gerado para uma partícula de massa $m_{\chi}=1000 \mathrm{GeV}$ aniquilando com contribuições de $90 \%$ do canal $b \bar{b}$ e $10 \% \tau^{+} \tau^{-}$. Os pontos correspondem aos dados da referência (21) e a curva azul indica a função aproximada.

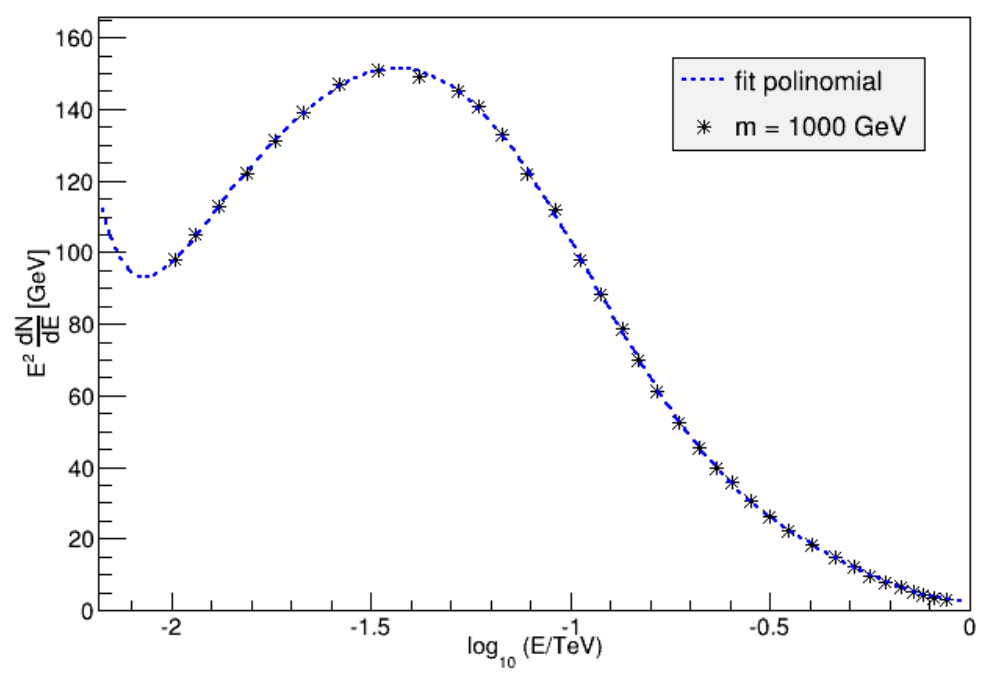

Fonte: Elaborada pela autora. 
Figura 5.7 - Curvas de limite superior de seção de choque pela massa da partícula derivados para Draco (curva azul) e Segue 1 (curva vermelha). As regiões hachuradas representam incertezas de $1 \sigma$. As linhas tracejadas verde e roxa indicam, respectivamente, o limite da seção de choque para Draco e o ruído de fundo de raios gama produzidas pelo FERMI-LAT (54).

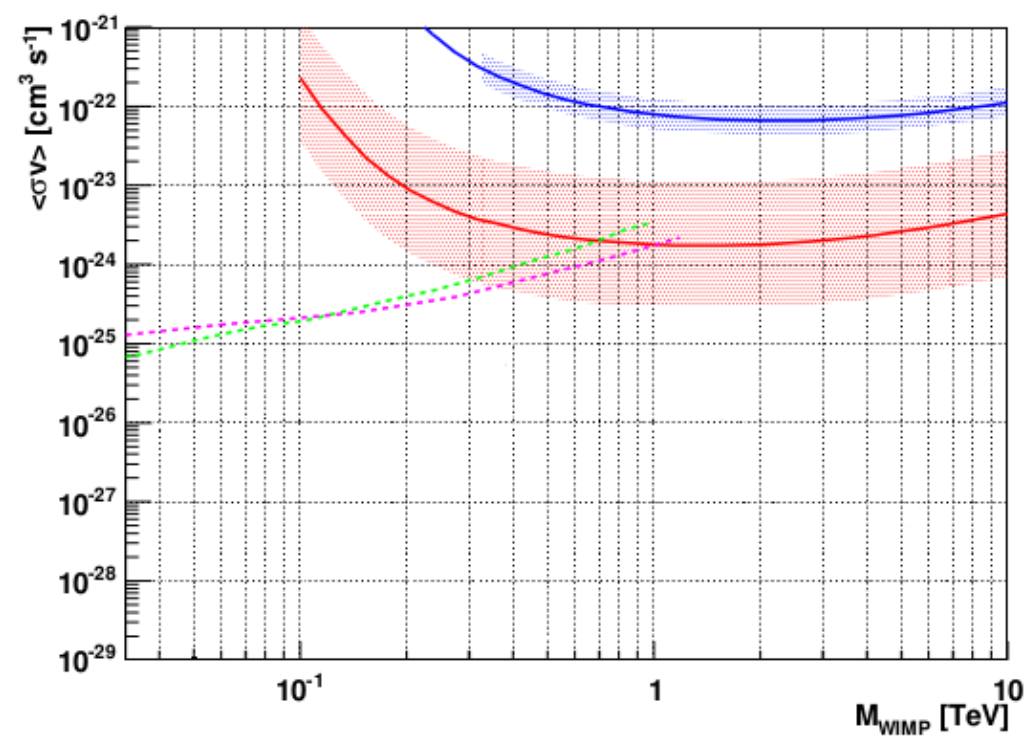

Fonte: Adaptada de WOOD. (21)

Tabela 5.6 - Valores obtidos para o limite superior da seção de choque com a implementação do algoritmo em comparação com os dados do VERITAS (21) para a galáxia $d S p h$ Draco.

\begin{tabular}{ccc}
\hline massa $[\mathrm{TeV}]$ & $\langle\sigma v\rangle_{\text {calculado }}\left[\mathrm{cm}^{3} \mathrm{~s}^{-1}\right]$ & $\left\langle\sigma v>_{\text {referência }}\left[\mathrm{cm}^{3} \mathrm{~s}^{-1}\right]\right.$ \\
\hline 0.316 & $1.5 \cdot 10^{-22}$ & $2.5 \cdot 10^{-22}$ \\
1 & $8.3 \cdot 10^{-23}$ & $7.0 \cdot 10^{-23}$ \\
3.16 & $1.1 \cdot 10^{-22}$ & $7.0 \cdot 10^{-23}$ \\
10 & $3.2 \cdot 10^{-22}$ & $1.0 \cdot 10^{-22}$ \\
\hline
\end{tabular}

Fonte: Elaborado pela autora.

Tabela 5.7 - Valores obtidos para o limite superior da seção de choque com a implementação do algoritmo em comparação com os dados do VERITAS (21) para a galáxia $d S p h$ Segue I.

\begin{tabular}{ccc}
\hline massa $[\mathrm{TeV}]$ & $\langle\sigma v\rangle_{\text {calculado }}\left[\mathrm{cm}^{3} \mathrm{~s}^{-1}\right]$ & $\langle\sigma v\rangle_{\text {referência }}\left[\mathrm{cm}^{3} \mathrm{~s}^{-1}\right]$ \\
\hline 0.316 & $3.7 \cdot 10^{-24}$ & $3.8 \cdot 10^{-24}$ \\
1 & $2.0 \cdot 10^{-24}$ & $2.0 \cdot 10^{-24}$ \\
3.16 & $2.8 \cdot 10^{-24}$ & $2.0 \cdot 10^{-24}$ \\
10 & $2.9 \cdot 10^{-24}$ & $4.5 \cdot 10^{-24}$ \\
\hline
\end{tabular}

Fonte: Elaborado pela autora.

Propagamos o erro da aproximação para todas as medidas de Draco e Segue I, porém para expressarmos o erro total no valor final da seção de choque precisamos do valor do desvio de 
$J$. Na referência (21) é citado o efeito final do erro em $J$ para os valores de limite superior da seção de choque, mas não é apresentado seu valor. Por esse motivo, não foi possível realizar a propagação de erro total nos valores obtidos pela implementação.

Para Draco, é estabelecido um erro no resultado final de $40 \%$. Segue I é referenciada a outras galáxias em um conjunto de erro finais variando de um fator 2 a 5 do resultado. Os erros nos resultados de limite superior de seção de choque na referência (21) são referentes a incertezas no cálculo do fator $J$. Como a propagação dos erros de aproximação retornam diferenças apenas a partir de $\approx 10^{-4}$, devemos considerar que a propagação do erro em $J$ dominaria o resultado sobre o da aproximação. Dessa forma, devemos admitir o mesmo erro sistemático nos valores obtidos com a implementação.

\subsection{Conclusão sobre as análises}

De uma forma geral, para as duas análises realizadas, as diferenças comparativas nos valores obtidos e nos valores das referências são aceitáveis por algumas razões.

Primeiramente, devemos considerar os limites de integração quando realizamos o cálculo da integral do perfil de produção de raios gama por aniquilação. Os valores das integrais são sensíveis a pequenas variações de energia $(\delta E \approx 0.05 \mathrm{TeV})$ pois as funções ajustadas envolvem variáveis logarítmicas. Assim, uma variação dessa magnitude no valor de energia de limiar, já proporcionaria um erro considerável no resultado final. Nas duas análises, as energias de limiar de cada telescópio não são apresentadas com essa precisão, portanto o cálculo desse possível desvio não pôde ser realizado.

Um outro fator a ser considerado é a sensibilidade do valor do limite superior de seção de choque quando consideramos diferentes telescópios para um mesmo modelo de partícula. Por exemplo, comparemos o limite superior na seção de choque de aniquilação do neutralino com massa $m=1 \mathrm{TeV}$ para a fonte Draco da referência (50) com o obtido na análise do VERITAS para o mesmo modelo. Na referência (50), o fator astrofísico equivale a $J \approx$ $5 \cdot 10^{12} \mathrm{TeV}^{2} \mathrm{~cm}^{-5} \mathrm{sr}$ e na análise do VERITAS $J \approx 4.71 \cdot 10^{11} \mathrm{TeV}^{2} \mathrm{~cm}^{-5} \mathrm{sr}$. Podemos considerar a diferença dos fatores em aproximadamente uma ordem de grandeza. No valor final da seção de choque isso corresponderia a $10^{-1}$. Porém, na referência (50), o limite superior na seção de choque para esse modelo corresponde a $\langle\sigma v\rangle \approx 1.9 \cdot 10^{-21} \mathrm{~cm}^{3} \mathrm{~s}^{-1}$ e na análise do VERITAS $\langle\sigma v\rangle \approx 7.0 \cdot 10^{-23} \mathrm{~cm}^{3} \mathrm{~s}^{-1}$. A explicação óbvia para o desvio provém do fato de serem diferentes telescópios, porém, evidencia a sensibilidade do cálculo do 
limite superior da seção de choque quando consideramos diferentes energias de limiar e perfis de produção de raios gama. 


\section{Previsão do limite da seção de choque de aniquilação medido pelo CTA}

Com a concordância dos resultados obtidos com os dados do VERITAS e MAGIC, pudemos estabelecer uma base de entendimento sobre o cálculo da seção de choque de aniquilação. Com isso, passamos para a resposta de simulação do CTA para fontes extensas a partir das ferramentas disponíveis e implementadas por Daniel Mazin (IFAE), Emma de Oña (MPIK) e Giovanna Pedaletti (IEEC-CSIC) para os membros da colaboração do CTA.

O código implementado para análise de fontes extensas depende de dois fatores principais de especificação do usuário. O primeiro deles é referente à configuração do telescópio, ou seja, a especificação do arranjo e do tempo de observação. Isso é feito a partir dos arquivos de resposta disponíveis também para a colaboração do CTA que contem informações como a área efetiva da configuração, resolução de energia, resolução angular, entre outros. O segundo fator é relacionado a características da medida. Entre algumas das variáveis possíveis de mudança, as principais são referentes a exigências estatísticas como significância, porcentagem de ruído de fundo considerado e número mínimo de eventos por medida.

Uma vez que definimos essas características para a detecção, podemos focar no argumento de entrada do programa que nada mais é do que a expressão de fluxo da fonte dependente da energia (equação 3.12). Com essas características e expressões bem definidas, o programa então gera o gráfico correspondente ao fluxo diferencial da fonte, especificando os pontos de detecção (se houver) e o fluxo integrado da curva correspondente. Na figura 6.1, podemos ver um exemplo do gráfico gerado para uma curva correspondente a $10 \%$ do fluxo da nebulosa de Carangueijo.

Na próxima seção detalharemos o funcionamento do programa de simulação focando nos primeiros resultados obtidos referentes às fontes e propriedades da referência (22). 
Figura 6.1 - Simulação de resposta do CTA de fluxo diferencial por energia de uma fonte emitindo $10 \%$ do fluxo correspondente a nebulosa de Carangueijo. À direita, podemos ver o mapeamento da emissão da fonte em questão.
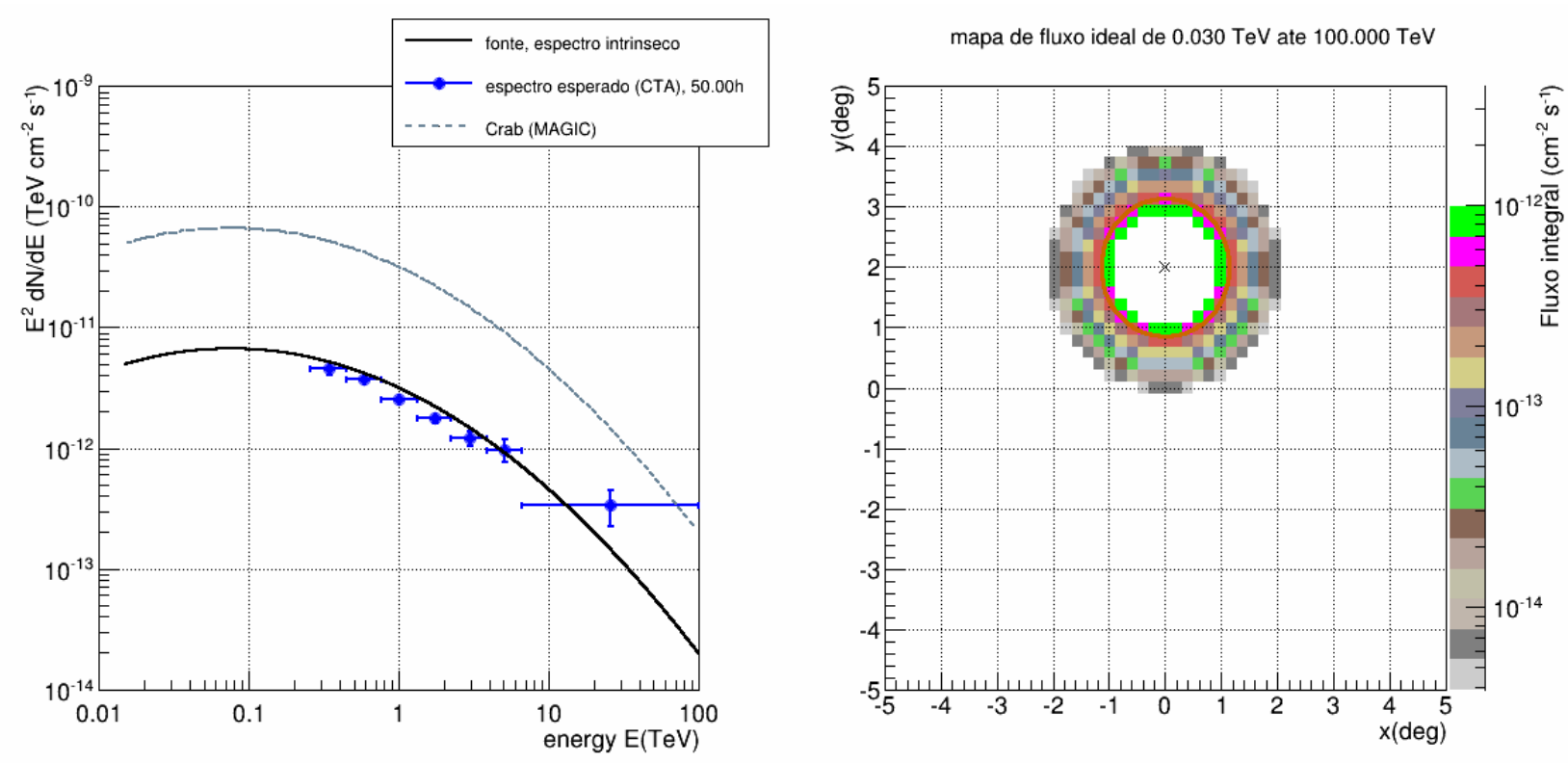

Fonte: Elaborada pela autora.

\subsection{Programa de Simulação e Primeiros Resultados}

Para os primeiros resultados obtidos com a implementação do CTA, escolhemos trabalhar com três fontes mencionadas na referência (22), que são as galáxias Sculptor, Segue 1 e Ursa Minor. Para obtermos as respectivas expressões de fluxo, precisamos determinar os fatores astrofísicos $J$ e as funções que definem as taxas de produção de raios gama por aniquilação $\left(\frac{\mathrm{d} N_{\gamma}}{\mathrm{d} E}\right)$.A tabela 6.1 apresenta os valores dos fatores astrofísicos para cada uma das fontes.

Tabela 6.1 - Fatores astrofísicos $J$ correspondentes a cada fonte.

\begin{tabular}{ccc}
\hline Fonte & $J\left(\mathrm{GeV}^{2} \mathrm{~cm}^{-5} \mathrm{sr}\right)$ & Perfil \\
\hline Sculptor & $8.9 \cdot 10^{17}$ & NFW \\
Segue 1 & $1.7 \cdot 10^{19}$ & Einasto \\
Ursa Minor & $2.2 \cdot 10^{18}$ & NFW \\
\hline
\end{tabular}

Fonte: Adaptado de DORO. (22)

Para essas fontes, os perfis de produção de raios gama por aniquilação, utilizamos os métodos e equações da referência (66). Além da disponibilização das equações analíticas, também foi possível obter as implementações para geração das curvas dependentes da massa da partícula. Dessa forma, com o software Mathematica (67), pudemos definir curvas que 
Figura 6.2 - Curva de $x^{1.5} \frac{\mathrm{d} N}{\mathrm{~d} x}$ por $x$, onde $x=\frac{E}{m_{\chi}}$, para a massa de $2 \mathrm{TeV}$ gerada com Mathematica.

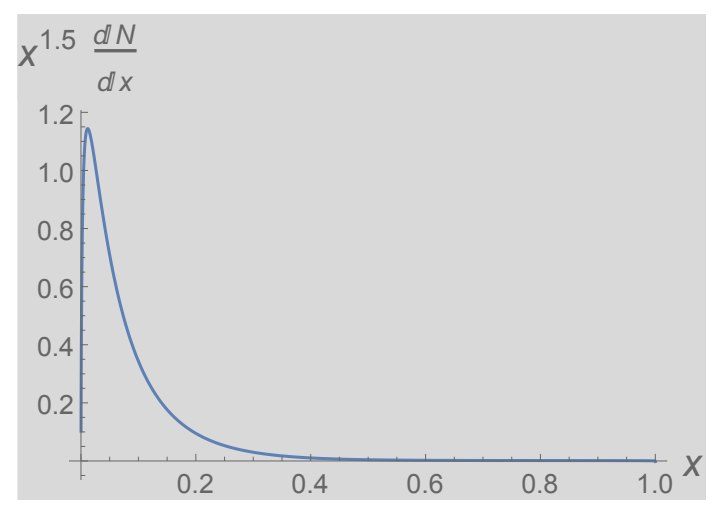

Fonte: Elaborada pela autora.

descrevem a taxa de produção de raios gama.

Geramos curvas para 8 massas diferentespara o canal de aniquilação $b \bar{b}$. Podemos ver na figura 6.2 um dos gráficos gerados pelo Mathematica para uma massa de $2 \mathrm{TeV}$. O eixo das ordenadas corresponde a $x^{1.5} \frac{d N}{d x}$ e o eixo das abscissas a $x$, onde $x=\frac{E}{m_{\chi}}$.

Uma vez com as curvas descritivas, podemos aproximar uma expressão para $\frac{d N_{\gamma}}{d E}$ dependente da energia. O processo para obter essa expressão foi análogo ao descrito no capítulo anterior: extraímos pontos da curva e implementamos um código para ajustar uma função e integrá-la em energia. Todas as funções ajustadas foram parametrizadas por um polinômio de terceiro grau da seguinte forma,

$$
\log _{10}\left(x^{1.5} \frac{\mathrm{d} N}{\mathrm{~d} x}\right)=p_{0}+p_{1} \cdot \log _{10} x+p_{2} \cdot\left(\log _{10} x\right)^{2}+p_{3} \cdot\left(\log _{10} x\right)^{3},
$$

onde $x=\frac{E}{m_{\chi}}$.

A tabela 6.2 contém os valores dos parâmetros $p_{0}, p_{1}, p_{2}$ e $p_{3}$ para cada função aproximada gerada para diferentes massas da partícula de matéria escura.

Para completarmos a expressão de fluxo diferencial de cada fonte, vamos focar primeiramente da expressão de $\frac{\mathrm{d} N}{\mathrm{~d} x}$. Como todas as funções aproximadas são polinômios de terceiro grau, diremos que:

$$
\begin{aligned}
\log _{10}\left(x^{1.5} \frac{\mathrm{d} N}{\mathrm{~d} x}\right) & =p_{0}+p_{1} \cdot \log _{10} x+p_{2} \cdot\left(\log _{10} x\right)^{2}+p_{3} \cdot\left(\log _{10} x\right)^{3} \\
x^{1.5} \frac{\mathrm{d} N}{\mathrm{~d} x} & =10^{p_{0}+p_{1} \cdot \log _{10} x+p_{2} \cdot\left(\log _{10} x\right)^{2}+p_{3} \cdot\left(\log _{10} x\right)^{3}} \\
\frac{\mathrm{d} N}{\mathrm{~d} x} & =\frac{1}{x^{1.5}} 10^{p_{0}+p_{1} \cdot \log _{10} x+p_{2} \cdot\left(\log _{10} x\right)^{2}+p_{3} \cdot\left(\log _{10} x\right)^{3}}
\end{aligned}
$$


Tabela 6.2 - Valores dos parâmetros do polinômio de terceiro grau para cada curva correspondente a uma dada massa.

\begin{tabular}{ccccc}
\hline massa $[\mathrm{TeV}]$ & $p_{0}$ & $p_{1}$ & $p_{2}$ & $p_{3}$ \\
\hline 0.8 & -3.621 & -5.505 & -2.763 & -0.461 \\
1 & -2.881 & -3.354 & -0.993 & -0.027 \\
2 & -2.768 & -2.860 & -0.594 & 0.055 \\
2.5 & -2.900 & -3.083 & -0.719 & 0.031 \\
5 & -2.901 & -3.254 & -0.996 & -0.063 \\
10 & -2.910 & -3.211 & -0.962 & -0.060 \\
15 & -2.820 & -3.069 & -0.891 & -0.047 \\
20 & -3.320 & -4.264 & -1.740 & -0.230 \\
\hline
\end{tabular}

Fonte: Elaborado pela autora.

Como $x=\frac{E}{m_{\chi}}$, note que

$$
\begin{aligned}
& \frac{\mathrm{d} N}{\mathrm{~d} x}=\frac{\mathrm{d} N}{\mathrm{~d} E} \cdot \frac{\mathrm{d} E}{\mathrm{~d} x}, \\
& \frac{\mathrm{d} N}{\mathrm{~d} x}=m_{\chi} \cdot \frac{\mathrm{d} N}{\mathrm{~d} E} .
\end{aligned}
$$

Logo, da equação 6.4

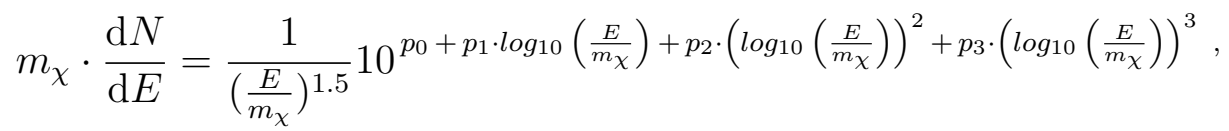

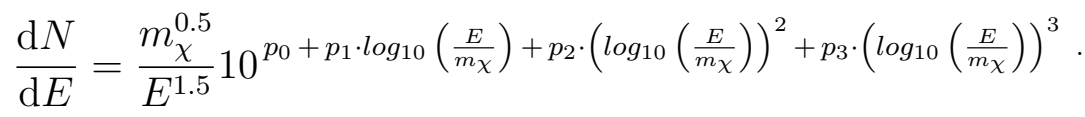

Assim para uma dada fonte com um fator $J$ específico, supondo uma massa para as partículas de matéria escura, a partir da equação 3.12 teremos a seguinte expressão:

$$
\begin{aligned}
& \frac{\mathrm{d} \Phi}{\mathrm{d} E}(\Delta \Omega)=\frac{<\sigma v>}{2 m_{\chi}^{2}} J \cdot \frac{m_{\chi}^{0.5}}{E^{1.5}} 10^{p_{0}+p_{1} \cdot \log _{10}\left(\frac{E}{m_{\chi}}\right)+p_{2} \cdot\left(\log _{10}\left(\frac{E}{m_{\chi}}\right)\right)^{2}+p_{3} \cdot\left(\log _{10}\left(\frac{E}{m_{\chi}}\right)\right)^{3}}, \\
& \frac{\mathrm{d} \Phi}{\mathrm{d} E}(\Delta \Omega)=\frac{<\sigma v>J}{2} \cdot \frac{1}{\left(m_{\chi} E\right)^{1.5}} 10^{p_{0}+p_{1} \cdot \log _{10}\left(\frac{E}{m_{\chi}}\right)+p_{2} \cdot\left(\log _{10}\left(\frac{E}{m_{\chi}}\right)\right)^{2}+p_{3} \cdot\left(\log _{10}\left(\frac{E}{m_{\chi}}\right)\right)^{3} .}
\end{aligned}
$$

Note agora que para definirmos a expressão de fluxo de raios gama exata da fonte, é preciso determinar o valor exato da seção de choque de aniquilação $\langle\sigma v\rangle$ da partícula de 
matéria escura. Como essa informação não está disponível, devemos focar nos limites do valor da seção de choque. Dessa forma, podemos expressar o valor da seção de choque dependente da massa da partícula e derivar limites para essas duas propriedades.

Para essa finalidade, consideramos dois pontos importantes sobre a simulação de detecção:

1. Queremos determinar o fluxo mínimo possível de detecção de cada fonte, para que possamos limitar o valor de seção de choque e de massa da partícula.

2. E devemos considerar que o cálculo efetuado pelo programa de simulação sobre os pontos de detecção carrega informação estatística em cada intervalo de energia do gráfico.

Para determinar o fluxo integral mínimo possível de ser detectado de cada fonte, minimizamos o valor de expressão de fluxo diferencial definida na equação 6.10. Ao executar o programa de resposta de simulação do CTA, obtemos o valor do fluxo integral dada a expressão de fluxo diferencial que determinamos.

Observe que a notação do programa para fluxo diferencial corresponde a $E^{2} \frac{\mathrm{d} N}{\mathrm{~d} E}$ que não representa a mesma expressão para a taxa de produção de raios gama por aniquilação descrita até então. Utilizaremos aqui o termo $\frac{\mathrm{d} N}{\mathrm{~d} E}$ somente para a produção de raios gama por aniquilação de partícula de matéria escura, com excessão apenas aos gráficos gerados pelo programa, os quais estarão bem esclarecidos nas respectivas legendas. A tabela 6.3 mostra os valores de massa e de fluxo integral mínimo para a fonte Sculptor $\left(J=8.9 \cdot 10^{11} \mathrm{TeV}^{2} \mathrm{~cm}^{-5} \mathrm{sr}\right)$ considerando uma detecção com significância $5 \sigma$, mínimo de 10 eventos por intervalo de energia e com $0.05 \%$ de ruído de fundo. A figura 6.3 mostra as curvas correspondentes aos dados da tabela 6.3

Tabela 6.3 - Valores de massa $m_{\chi}(\mathrm{TeV})$ e fluxo integral mínimo da fonte Sculptor $\Phi \mathrm{cm}^{-2} \mathrm{~s}^{-1}$.

\begin{tabular}{cc}
\hline$m_{\chi}(\mathrm{TeV})$ & $\Phi\left(\mathrm{cm}^{-2} \mathrm{~s}^{-1}\right)$ \\
\hline 0.8 & $1.55 \cdot 10^{-9}$ \\
1.0 & $1.31 \cdot 10^{-9}$ \\
2.0 & $4.32 \cdot 10^{-10}$ \\
2.5 & $3.80 \cdot 10^{-10}$ \\
5.0 & $1.24 \cdot 10^{-10}$ \\
10.0 & $5.91 \cdot 10^{-11}$ \\
15.0 & $4.76 \cdot 10^{-11}$ \\
20.0 & $4.38 \cdot 10^{-11}$ \\
\hline
\end{tabular}

Fonte: Elaborado pela autora.

Com a finalidade de encontrarmos uma função de $\langle\sigma v\rangle$ por $m_{\chi}$ determinada com a 
Figura 6.3 - Curvas de fluxo diferencial mínimo detectável por energia geradas para as 8 massas diferentes, para a fonte Sculptor $\left(J=8.9 \cdot 10^{11} \mathrm{TeV}^{2} \mathrm{~cm}^{-5} \mathrm{sr}\right)$ com significância $5 \sigma$, mínimo de 10 eventos por intervalo de energia e com $0.05 \%$ de ruído de fundo. Da esquerda para a direita, curvas geradas com massa de $0.8 \mathrm{TeV}, 1 \mathrm{TeV}, 2 \mathrm{TeV}$ e $2.5 \mathrm{TeV}$, e abaixo com massas de $5 \mathrm{TeV}, 10 \mathrm{TeV}, 15 \mathrm{TeV}$ e $20 \mathrm{TeV}$
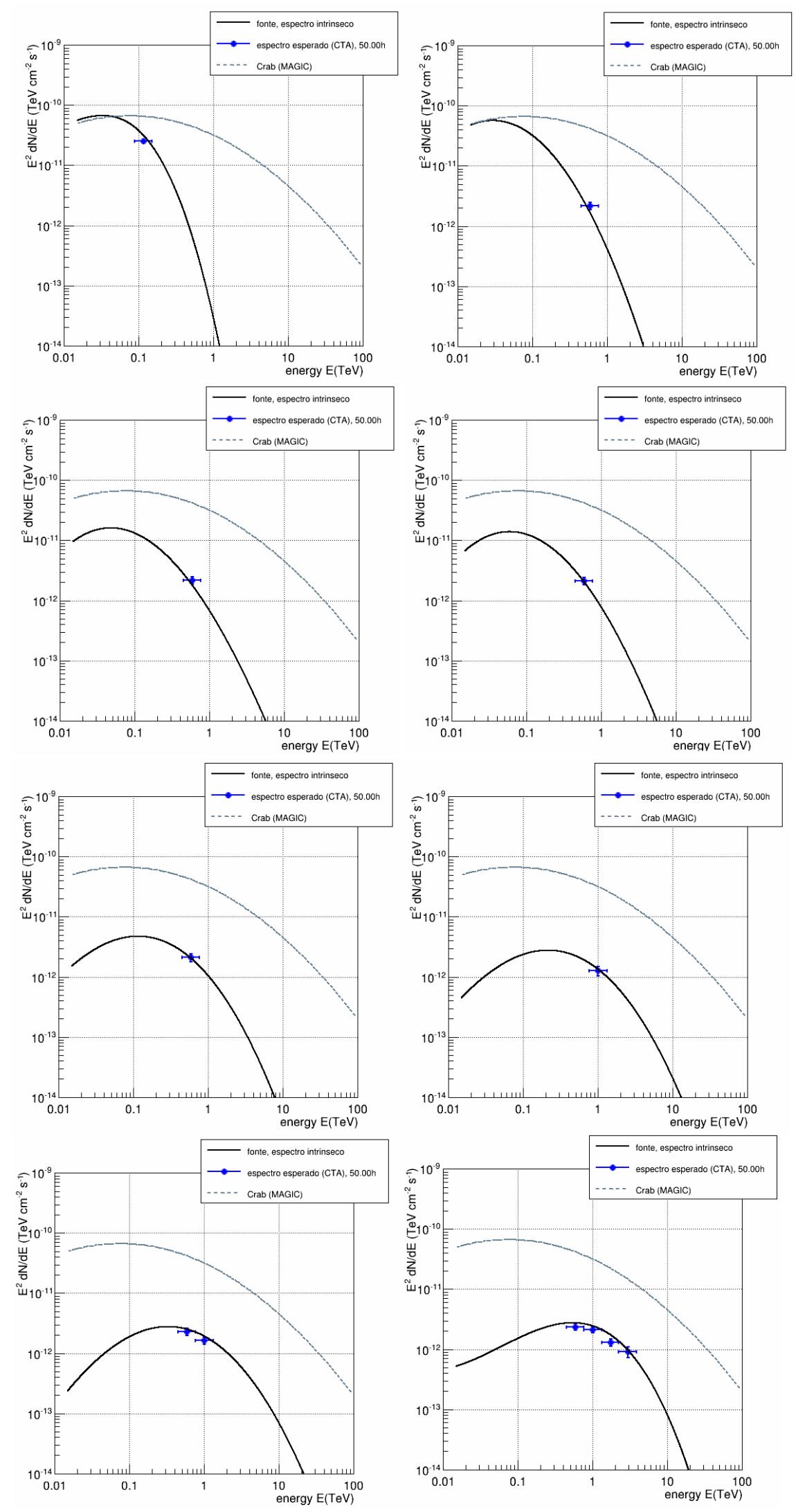

Fonte: Elaborada pela autora. 
possibilidade de detecção do CTA para uma dada fonte, implementamos um código para integração da equação 6.10 em energia a partir do valor de energia de limiar até o valor de energia equivalente a $200 \mathrm{TeV}$. Como a energia máxima do CTA é calculada em aproximadamente $100 \mathrm{TeV}$, os valores de fluxo até $200 \mathrm{TeV}$ são nulos. Então,

$$
\int_{E_{\mathrm{th}}}^{200} \frac{\mathrm{d} \Phi}{\mathrm{d} E}(\Delta \Omega) \mathrm{d} E=\frac{<\sigma v>\frac{1}{2 m_{\chi}^{2}}}{J} \cdot \int_{E_{\mathrm{th}}}^{200} \frac{\mathrm{d} N}{\mathrm{~d} E}
$$

Como $\Phi=\int_{E_{\text {th }}}^{200} \frac{\mathrm{d} \Phi}{\mathrm{d} E}(\Delta \Omega) \mathrm{d} E$, temos que

$$
\begin{gathered}
\Phi_{\text {real }}=\frac{\langle\sigma v>}{2 m_{\chi}^{2}} J \cdot \int_{E_{\text {th }}}^{200} \frac{\mathrm{d} N}{\mathrm{~d} E}, \\
\Rightarrow<\sigma v>=2 \Phi_{\text {real }} m_{\chi}^{2} \frac{1}{J} \cdot \frac{1}{\int_{E_{\mathrm{th}}}^{200} \frac{\mathrm{d} N}{\mathrm{~d} E}} .
\end{gathered}
$$

Dessa forma,

$$
<\sigma v>=2 \Phi_{\text {real }} m_{\chi}^{2} \frac{1}{J} \cdot\left(\int_{E_{\mathrm{th}}}^{200} \frac{1}{x^{1.5}} 10^{p_{0}+p_{1} \cdot \log _{10} x+p_{2} \cdot\left(\log _{10} x\right)^{2}+p_{3} \cdot\left(\log _{10} x\right)^{3}}\right)^{-1}
$$

Substituindo $x=\frac{E}{m_{\chi}}$,

$<\sigma v>=2 \Phi_{\text {real }} m_{\chi}^{2} \frac{1}{J} \cdot\left(\int_{E_{\text {th }}}^{200} \frac{1}{\left(\frac{E}{m_{\chi}}\right)^{1.5}} 10^{p_{0}+p_{1} \cdot \log _{10}\left(\frac{E}{m_{\chi}}\right)+p_{2} \cdot\left(\log _{10}\left(\frac{E}{m_{\chi}}\right)\right)^{2}+p_{3} \cdot\left(\log _{10}\left(\frac{E}{m_{\chi}}\right)\right)^{3}}\right)^{-1}$.

Com os valores das integrais e dos fluxos integrais da fonte obtidos com o programa de simulação do CTA, estabelecemos expressões de $\langle\sigma v\rangle$ dependentes da energia para uma massa específica. Essas expressões resultam nos pontos bem definidos da curva. Por fim, aproximamos uma função aos pontos definidos que descreve o comportamento de $\langle\sigma v\rangle$ pelo valor de massa $m_{\chi}$ da partícula.

Utilizamos como configuração do telescópio o arranjo E, com tempo de observação de $50 \mathrm{~h}$. Para as três fontes com os fatores $J$ definidos pela referência (22), produzimos resultados com significância $5 \sigma$, mínimo de 10 eventos por intervalo de energia e com $0.05 \%$ de ruído de fundo para o canal de aniquilação $b \bar{b}$. A tabela 6.5 contém os valores de massa e de seção de 
choque de aniquilação para cada uma das fontes obtidos com o procedimento descrito acima. Os erros relacionados foram propagados com os desvios no valor de $J$ descrito nas referências mencionadas em (22), sendo estas (24) para Sculptor e Ursa Minor e (46) para Segue 1. O erro de aproximação das curvas de $\frac{\mathrm{d} N_{\gamma}}{\mathrm{d} E}$ também foi considerado, porém sua magnitude é de apenas $\approx 10^{-2}$, o que resulta na dominância do erro de $J$ para o valor final de seção de choque de aniquilação.

Tabela 6.4 - Valores de seção de choque e respectiva massa para as fontes Sculptor, Ursa Minor e Segue I, partir dos dados em (22). Os resultados foram extraídos com significância $5 \sigma$, mínimo de 10 eventos por intervalo e ruído de fundo compondo $0.05 \%$ do sinal para o arranjo $\mathrm{E}$ com tempo de observação $T=50 \mathrm{~h}$.

\begin{tabular}{cccccc}
\hline \multicolumn{2}{c}{ Sculptor } & \multicolumn{2}{c}{ Ursa Minor } & \multicolumn{2}{c}{ Segue $\mathbf{1}$} \\
\hline$m_{\chi}(\mathrm{TeV})$ & $<\sigma v>\left(\mathrm{cm}^{3} \mathrm{~s}^{-1}\right)$ & $m_{\chi}(\mathrm{TeV})$ & $<\sigma v>\left(\mathrm{cm}^{3} \mathrm{~s}^{-1}\right)$ & $m_{\chi}(\mathrm{TeV})$ & $<\sigma v>\left(\mathrm{cm}^{3} \mathrm{~s}^{-1}\right)$ \\
\hline 0.8 & $7.0_{-0.6}^{+0.9} \cdot 10^{-22}$ & 0.8 & $2.7_{-1.3}^{+5.5} \cdot 10^{-22}$ & 0.8 & $3.4_{-0.2}^{+0.2} \cdot 10^{-23}$ \\
1.0 & $7.1_{-0.6}^{+0.9} \cdot 10^{-22}$ & 1.0 & $3.0_{-1.5}^{+6.1} \cdot 10^{-22}$ & 1.0 & $3.7_{-0.2}^{+0.2} \cdot 10^{-23}$ \\
2.0 & $4.0_{-0.1}^{+0.5} \cdot 10^{-22}$ & 2.0 & $1.8_{-0.9}^{+3.6} \cdot 10^{-22}$ & 2.0 & $2.3_{-0.1}^{+0.1} \cdot 10^{-23}$ \\
2.5 & $4.3_{-0.3}^{+0.6} \cdot 10^{-22}$ & 2.5 & $1.8_{-0.9}^{+3.6} \cdot 10^{-22}$ & 2.5 & $2.1_{-0.1}^{+0.1} \cdot 10^{-23}$ \\
5.0 & $3.3_{-0.3}^{+0.4} \cdot 10^{-22}$ & 5.0 & $1.3_{-0.7}^{+2.7} \cdot 10^{-22}$ & 5.0 & $1.7_{-0.1}^{+0.1} \cdot 10^{-23}$ \\
10.0 & $3.8_{-0.3}^{+0.5} \cdot 10^{-22}$ & 10.0 & $1.7_{-0.8}^{+3.5} \cdot 10^{-22}$ & 10.0 & $2.6_{-0.2}^{+0.2} \cdot 10^{-23}$ \\
15.0 & $5.8_{-0.5}^{+0.8} \cdot 10^{-22}$ & 15.0 & $1.9_{-0.8}^{+3.8} \cdot 10^{-22}$ & 15.0 & $4.3_{-0.3}^{+0.3} \cdot 10^{-23}$ \\
20.0 & $8.0_{-0.6}^{+1.1} \cdot 10^{-22}$ & 20.0 & $2.2_{-1.1}^{+4.5} \cdot 10^{-22}$ & 20.0 & $3.9_{-0.2}^{+0.2} \cdot 10^{-23}$ \\
\hline
\end{tabular}

Fonte: Elaborado pela autora.

As figuras $6.4,6.5$ e 6.6 mostram as funções definidas pelos pontos descritos na tabela juntamente com as curvas da referência (22) também derivadas com significância $5 \sigma$, mínimo de 10 eventos por intervalo de energia e com $0.05 \%$ de ruído de fundo para o canal de aniquilação $b \bar{b}$. A diferença entre as configurações reside nos tempos de observação: extraímos dados com $T=50 \mathrm{~h}$ e na referência as curvas correspondem a $T=100 \mathrm{~h}$. É possível notar a mudança com relação à sensitividade das propriedades como descrito no capítulo 4 de forma a validar os resultados obtidos.

\subsection{Resultados com fontes estudadas pelo FERMI- LAT}

Nessa seção focaremos nas fontes e respectivas propriedades estudadas pelo telescópio FERMI-LAT (39) na referência (23). Foram analisadas 25 galáxias anãs esferoidais no artigo em questão, das quais 18 estudamos com o programa de simulação do CTA. Na tabela 6.5 
Figura 6.4 - Curva da seção de choque por massa para a galáxia Sculptor com tempo de observação de $50 \mathrm{~h}$ (em vermelho) e com a curva $100 \mathrm{~h}$ apresentada na referência (22).

\section{Sculptor}

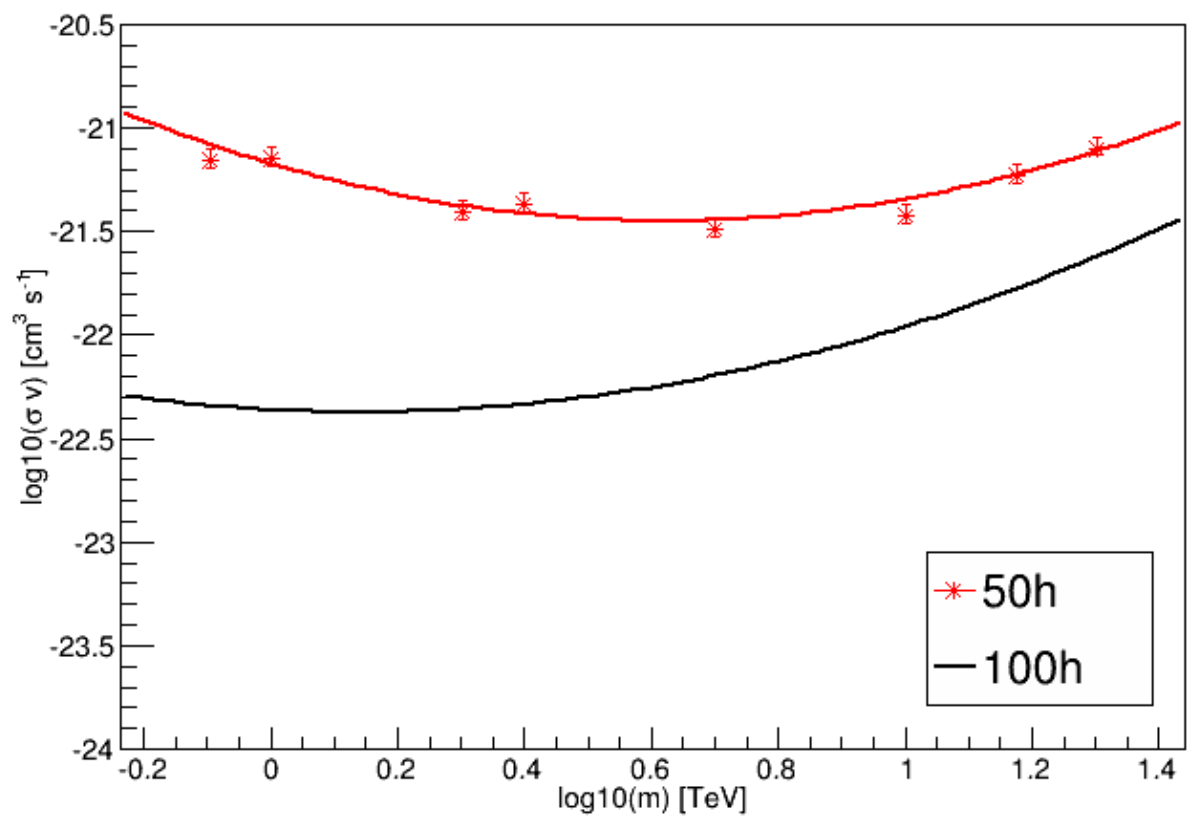

Fonte: Elaborada pela autora.

Figura 6.5 - Curva da seção de choque por massa para a galáxia Ursa Minor com tempo de observação de $50 \mathrm{~h}$ (em vermelho) e com a curva $100 \mathrm{~h}$ apresentada na referência (22).

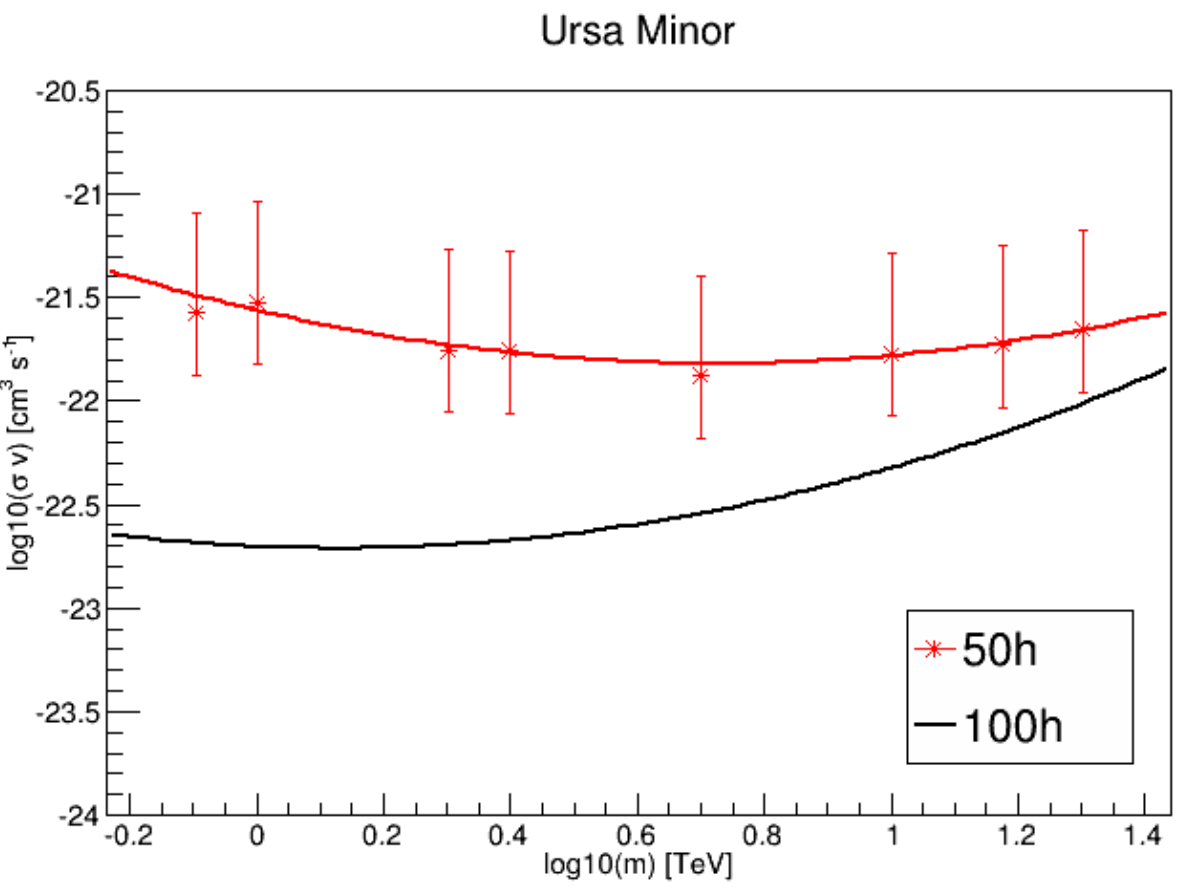

Fonte: Elaborada pela autora. 
Figura 6.6 - Curva da seção de choque por massa para a galáxia Segue I com tempo de observação de $50 \mathrm{~h}$ (em vermelho) e com a curva $100 \mathrm{~h}$ apresentada na referência (22).

Segue I

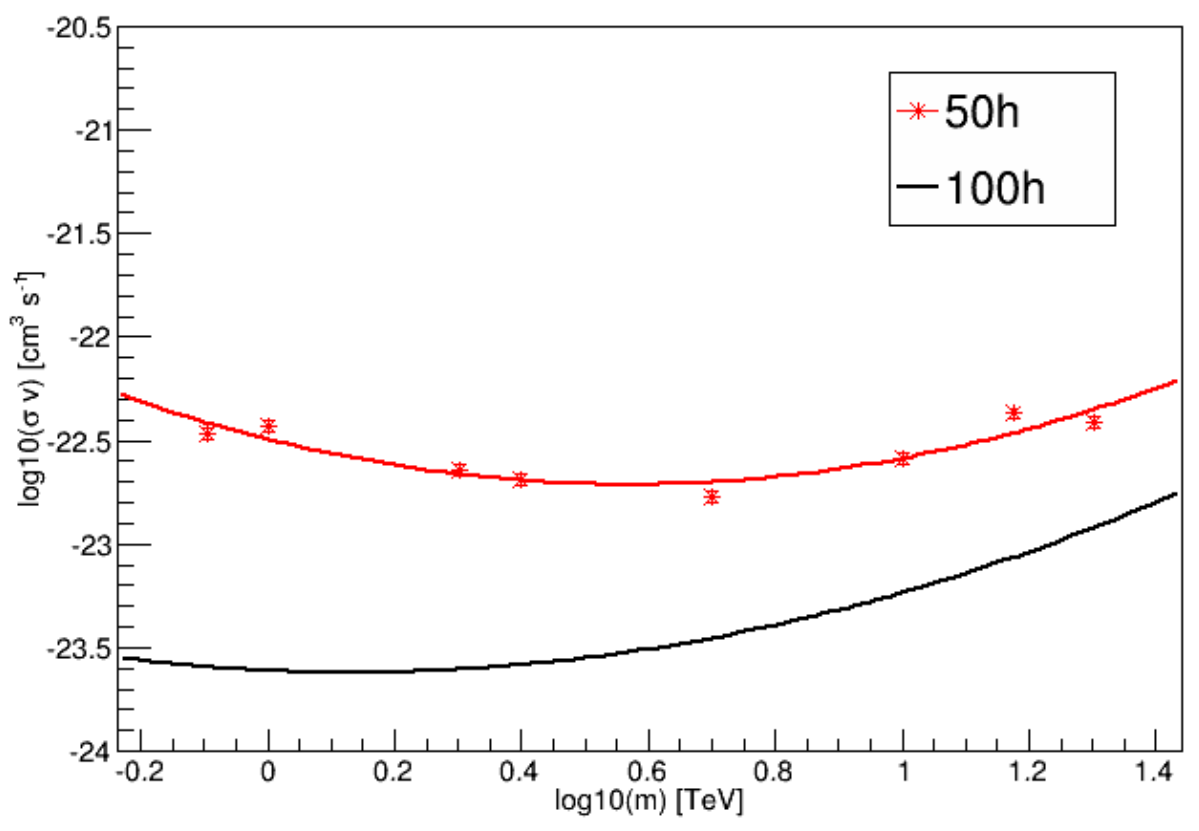

Fonte: Elaborada pela autora.

listamos as tais fontes com seus fatores astrofísicos $J$ e respectivos erros. Na tabela 6.6 listamo-as com seus nomes de catálogo através da referência (68).

Dividiremos essa seção em duas subseções principais. Primeiramente com as fontes definidas pelo FERMI-LAT, realizamos um estudo com relação a diferenças estatísticas de medidas de detecção e seu efeito sobre o valor final da seção de choque de aniquilação. Então, a partir dos limites superiores impostos no artigo (23), analisamos quais fontes poderão ter uma contribuição bastante significativa do CTA.

\subsubsection{Resultados com significância $3 \sigma$ e $5 \sigma$}

Para analisarmos o efeito das exigências estatísticas sobre as medidas, escolhemos duas configurações distintas:

1. Significância $3 \sigma$, mínimo de 7 eventos por intervalo de energia e $0.03 \%$ de ruído de fundo;

2. Significância $5 \sigma$, mínimo de 10 eventos por intervalo de energia e $0.05 \%$ de ruído de 
Tabela 6.5 - Fontes estudadas, seus fatores astrofísicos $J$ e respectivos erros.

\begin{tabular}{ccc}
\hline \multicolumn{3}{c}{ Fonte } \\
& $J_{-\Delta J}^{+\Delta J}\left(\mathrm{TeV}^{2} \mathrm{~cm}^{-5} \mathrm{sr}\right)$ \\
\hline Sculptor & Segue 1 & Ursa Minor \\
$3.98_{-1.34}^{+2.05} \cdot 10^{12}$ & $3.16_{-1.54}^{+3.01} \cdot 10^{13}$ & $6.31_{-2.24}^{+3.46} \cdot 10^{12}$ \\
Carina & Fornax & Leo I \\
$1.26_{-0.52}^{+0.88} \cdot 10^{12}$ & $1.58_{-0.60}^{+0.99} \cdot 10^{12}$ & $5.01_{-1.70}^{+2.58} \cdot 10^{11}$ \\
Leo II & Sextans & Bootes I \\
$3.98_{-1.35}^{+2.05} \cdot 10^{11}$ & $2.51_{-1.16}^{+2.16} \cdot 10^{12}$ & $6.31_{-2.51}^{+4.16} \cdot 10^{12}$ \\
& & \\
Canes Venatici I & Canes Venatici II & Coma Berenices \\
$5.01_{-2.26}^{+4.11} \cdot 10^{11}$ & $7.94_{-3.48}^{+6.18} \cdot 10^{11}$ & $1.00_{-0.44}^{+0.78} \cdot 10^{13}$ \\
Hercules & Leo IV & Ursa Major I \\
$1.26_{-0.55}^{+1.98} \cdot 10^{12}$ & $7.94_{-3.77}^{+7.20} \cdot 10^{11}$ & $1.995_{-0.85}^{+1.47} \cdot 10^{12}$ \\
Ursa Major II & Draco & Willman I \\
$1.995_{-0.95}^{+1.81} \cdot 10^{13}$ & $6.31_{-1.94}^{+2.81} \cdot 10^{12}$ & $1.26_{-0.64}^{+1.31} \cdot 10^{13}$ \\
\hline
\end{tabular}

Fonte: Adaptado de ACKERMANN. (23)

fundo.

A fim de limitar a capacidade de detecção do CTA para as duas configurações, mapeamos com o programa de simulação as sensitividades no valor de fluxo. Definimos expressões correspondentes a fluxos de raios gama de uma fonte. Realizamos as medidas com mais de uma expressão a fim de comprovar a não dependência da sensitividade com uma expressão de fluxo específica. Para encontrarmos a sensitividade, de forma análoga aos mínimos obtidos nas seções anteriores, em cada intervalo de energia observamos o valor mínimo da expressão que continha um ponto detectado pelo telescópio. Dessa forma, traçamos um perfil de valor de fluxo por energia representativo dos mínimos possíveis de serem detectados. As figuras 6.7 e 6.8 mostram, respectivamente, as sensitividades para as configurações de $3 \sigma$ e $5 \sigma$ e os testes para validação do perfil encontrado.

Para estudar os efeitos das diferentes configurações de $3 \sigma$ e $5 \sigma$ sobre as 18 fontes da referência (23) realizamos um procedimento análogo ao descrito na seção anterior. Para os perfis de produção de raios gama por aniquilação, utilizamos as parametrizações definidas na tabela 6.2 Para os valores de fluxos integrais, realizamos o mesmo procedimento de minimização. E a fim de caracterizar a função de seção de choque $<\sigma v>$ por massa $m_{\chi}$, integramos a equação 6.10 em energia. 
Figura 6.7 - Sensitividade correspondente à configuração de $3 \sigma$, número mínimo de eventos correspondente a 7 e ruído de fundo equivalente a $0.03 \%$ do sinal representada pela curva em verde. As retas em preto foram argumentos de entrada do programa a fim de validar a região de detecção com as características descritas pela sensitividade da curva verde.
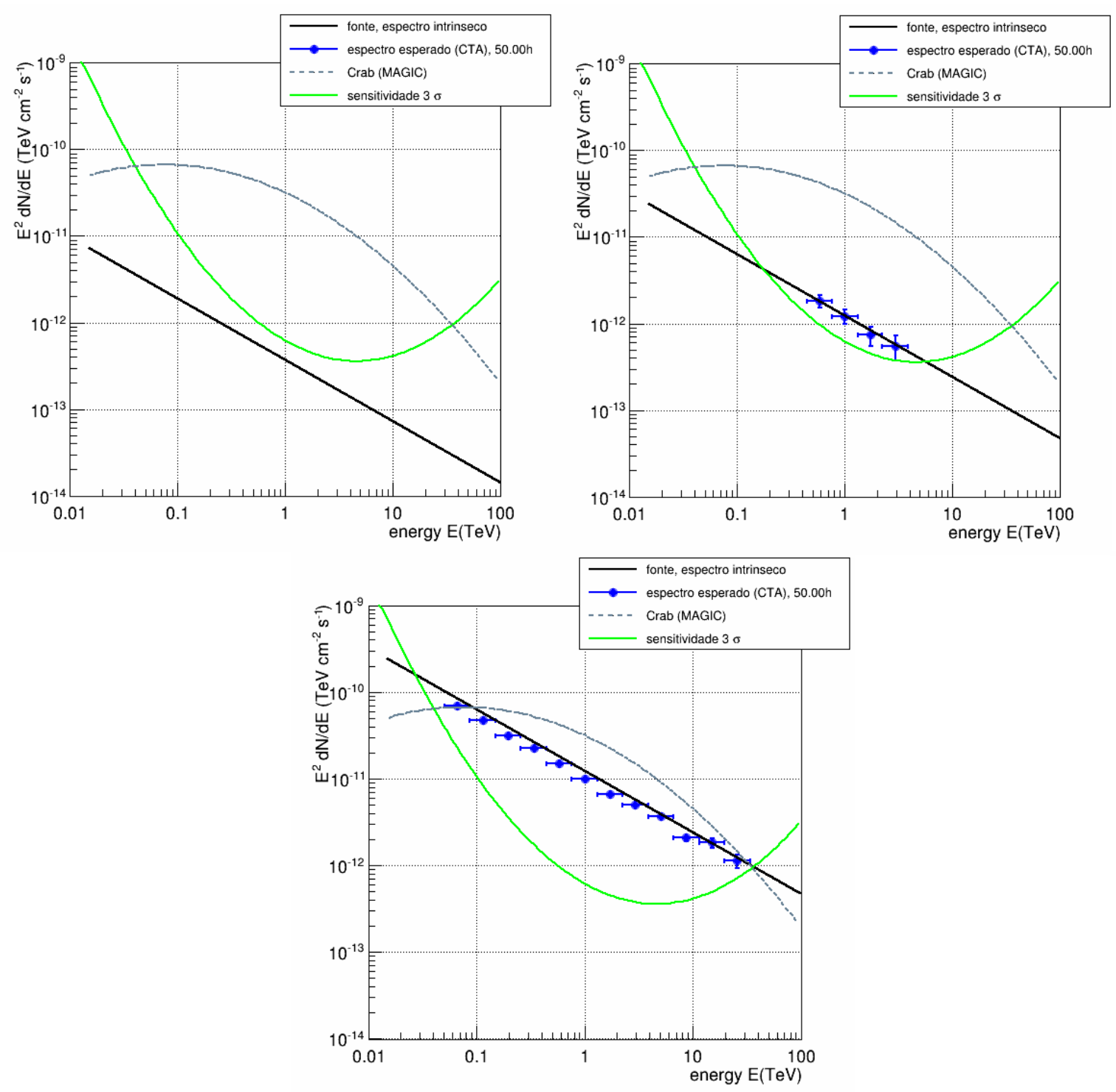

Fonte: Elaborada pela autora. 
Figura 6.8 - Sensitividade correspondente à configuração de $5 \sigma$, número mínimo de eventos correspondente a 10 e ruído de fundo equivalente a $0.05 \%$ do sinal representada pela curva em vermelho. Também testamos a validade da curva de sensitividade com as funções correspondentes as retas em preto.
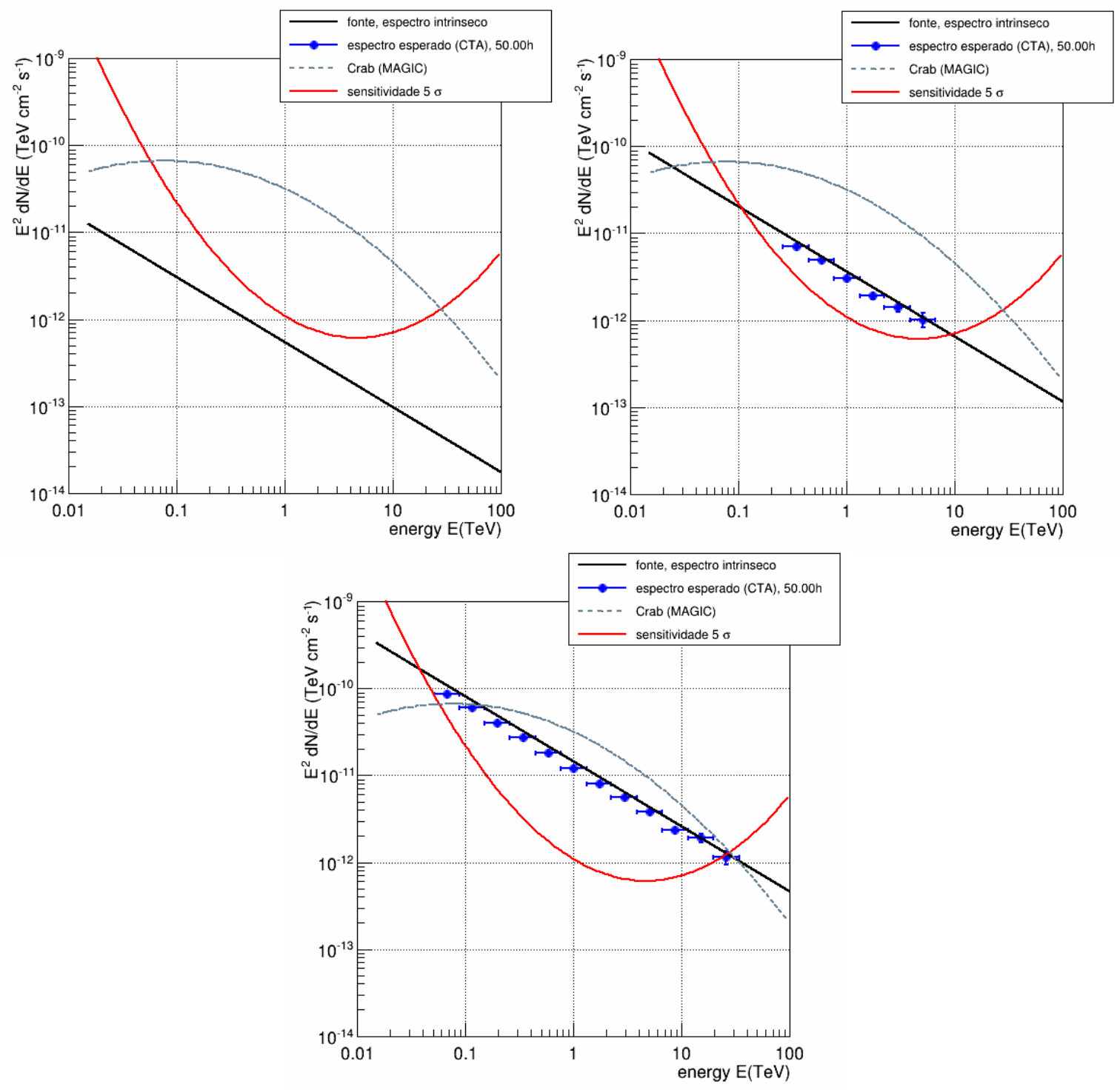

Fonte: Elaborada pela autora. 
Tabela 6.6 - Fontes e seus nomes por catálogo.

\begin{tabular}{cc}
\hline Fonte & Nome do Objeto \\
\hline Sculptor & RC2 A0057-33 \\
Segue I & LM2010 58 \\
Ursa Minor & RC2 A1508+67 \\
Carina & PGC 019441 \\
Fornax & RC2 A0237-34 \\
Leo I & RC1 A1006 \\
Leo II & PGC 034176 \\
Sextans & LEDA 088608 \\
Bootes I & LM2010 62 \\
Canes Venatici I & CVn I dSph \\
Canes Venatici II & SDSS J1257+3419 \\
Coma Berenices & LM2010 60 \\
Hercules & HERCULES DWARF SPHEROIDAL \\
Leo IV & LEO IV DWARF SPHEROIDAL \\
Ursa Major I & URSA MAJOR I DWARF \\
Ursa Major II & LM2010 57 \\
Draco & DRACO DWARF SPHEROIDAL \\
Willman I & SDSS J1049+5103 \\
\hline
\end{tabular}

Fonte: Elaborada pela autora.

As figuras 6.9 6.10 e 6.11 mostram os gráficos para as 18 fontes de (23) estudadas com o comparativo entre as duas configurações. A tabela 6.7 apresenta os valores dos pontos obtidos. Os erros nos valores de seção de choque foram resultado da propagação dos desvios dos fatores $J$ e dos desvios referentes à aproximação dos polinômio em $\frac{\mathrm{d} N_{\gamma}}{d E}$. Novamente, a dominância do erro permanece com os desvios de $J$ porque os desvios da aproximação são comparativamente pequenos.

É notável a diferença entre os resultados obtidos com significância $3 \sigma$ e $5 \sigma$ uma vez que essas exigências estatísticas tem o efeito de restringir ou abranger os valores obtidos. Para fontes com um fator astrofísico maior, podemos ver que os valores de seção de choque diminuem. A partir dos gráficos, podemos ver que para Segue I, por exemplo, considerando uma massa de $5 \mathrm{TeV}$, foram alcançados valores de $5.5 \cdot 10^{-24} \mathrm{~cm}^{3} \mathrm{~s}^{-1}$ com significância $3 \sigma$ e $9.2 \cdot 10^{-24} \mathrm{~cm}^{3} \mathrm{~s}^{-1}$ com significância $5 \sigma$.

Nesta seção, validamos o efeito das exigências estatísticas limitando valores possíveis de serem encontrados com o CTA. Mostramos também qual a possibilidade de caracterizar a partícula de matéria escura para as 18 fontes estudadas, considerando a sensitividade do telescópio para duas configurações diferentes. 
Figura 6.9 - Curvas de seção de choque de aniquilação por massa para as 18 fontes estudadas com duas configurações diferentes: significância $3 \sigma$, mínimo de eventos 7 e ruído de fundo $0.03 \%$ e significância $5 \sigma$, mínimo de eventos 10 e ruído de fundo $0.05 \%$. A legenda presente no gráfico da fonte Sculptor se aplica para todos os outros gráficos.
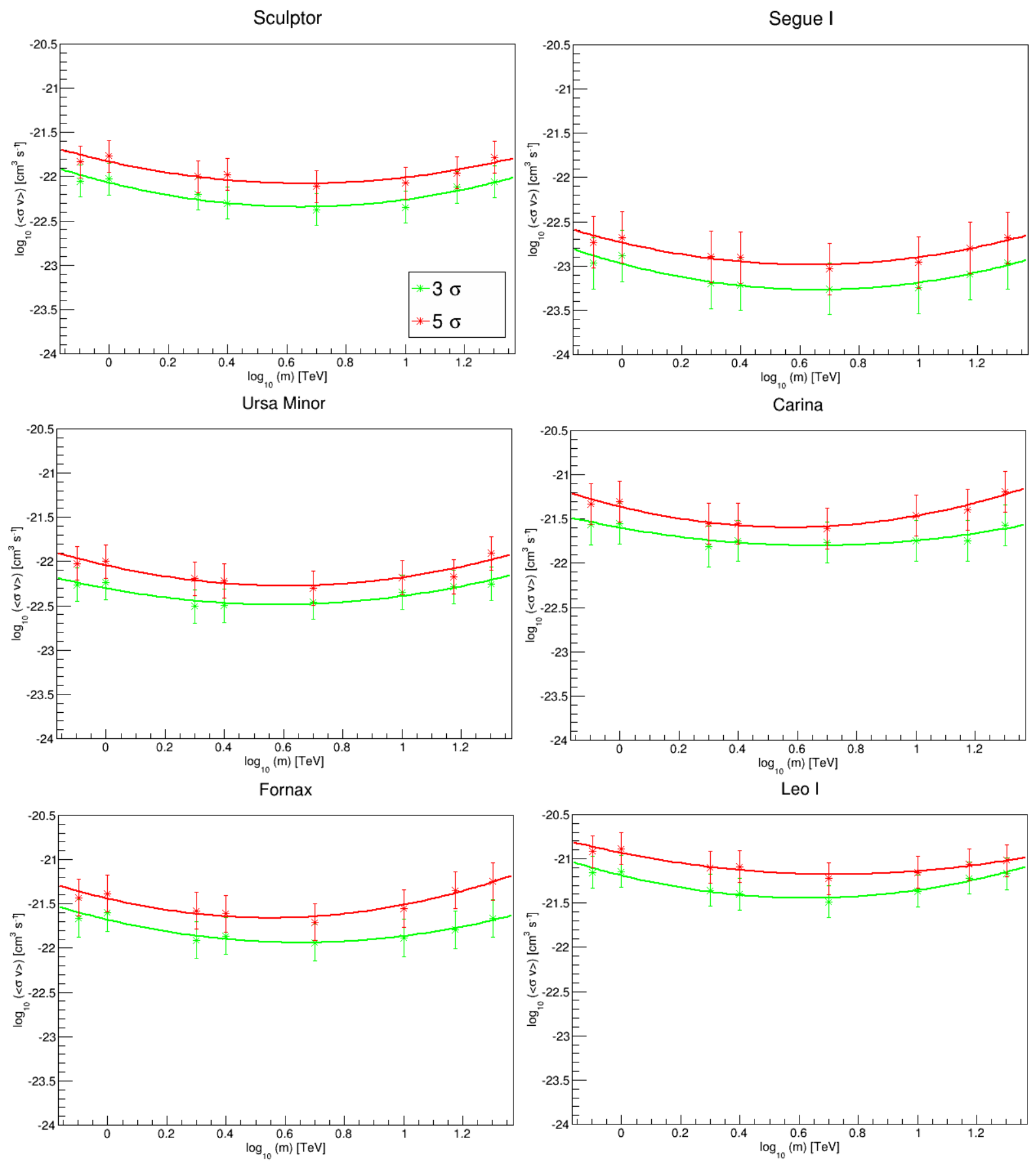

Fonte: Elaborada pela autora. 
Figura 6.10 - Curvas de seção de choque de aniquilação por massa para as 18 fontes estudadas com duas configurações diferentes: significância $3 \sigma$, mínimo de eventos 7 e ruído de fundo $0.03 \%$ e significância $5 \sigma$, mínimo de eventos 10 e ruído de fundo $0.05 \%$. A legenda presente no gráfico da fonte Sculptor se aplica para todos os outros gráficos.

Leo II
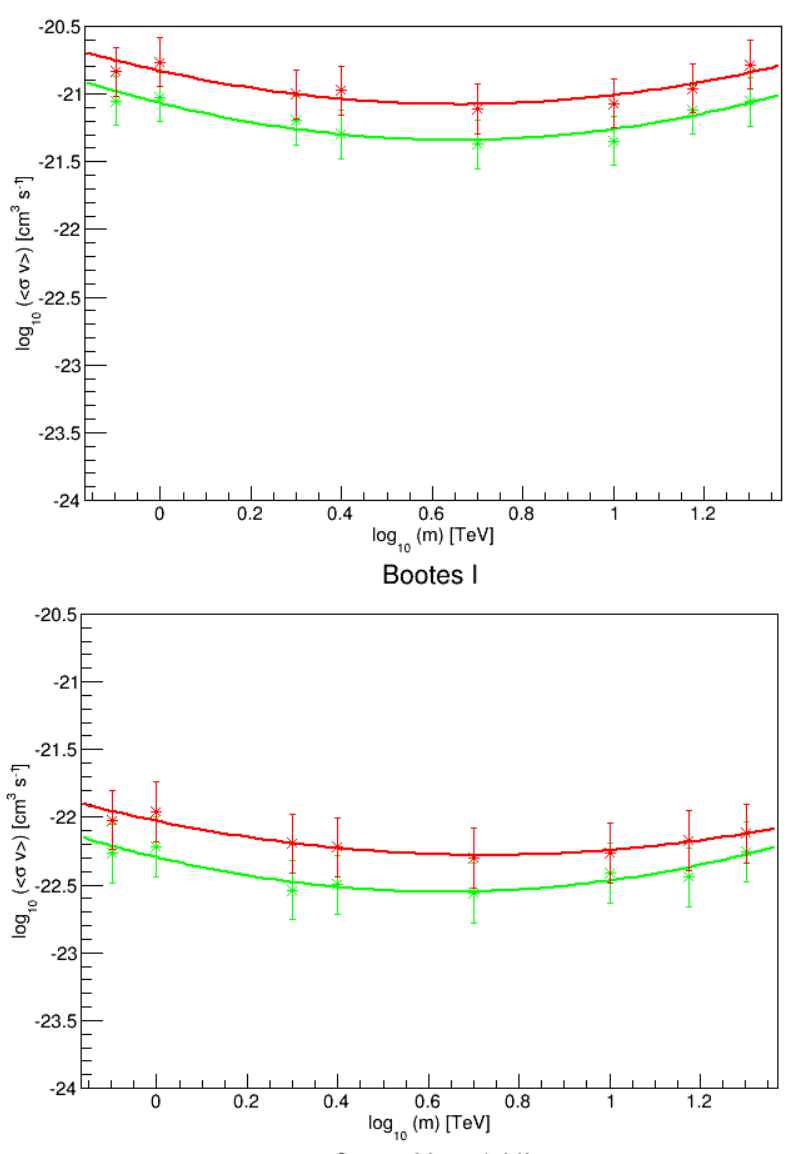

Canes Venatici II

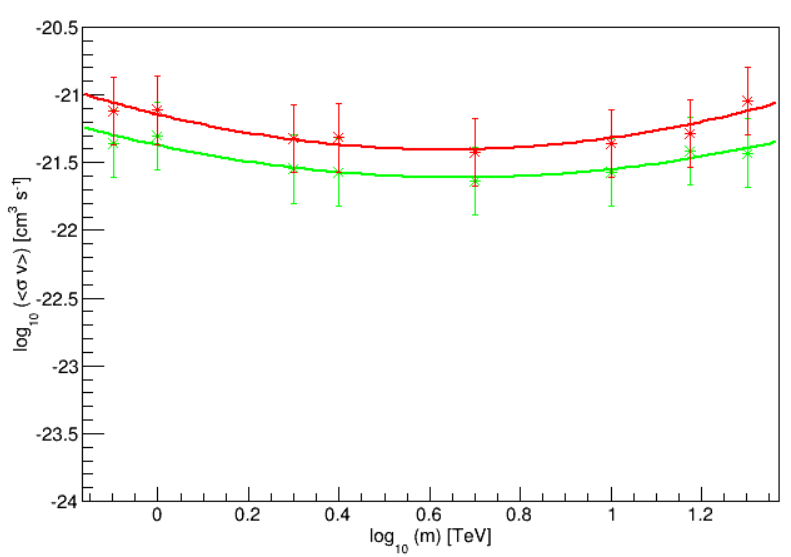

Sextans
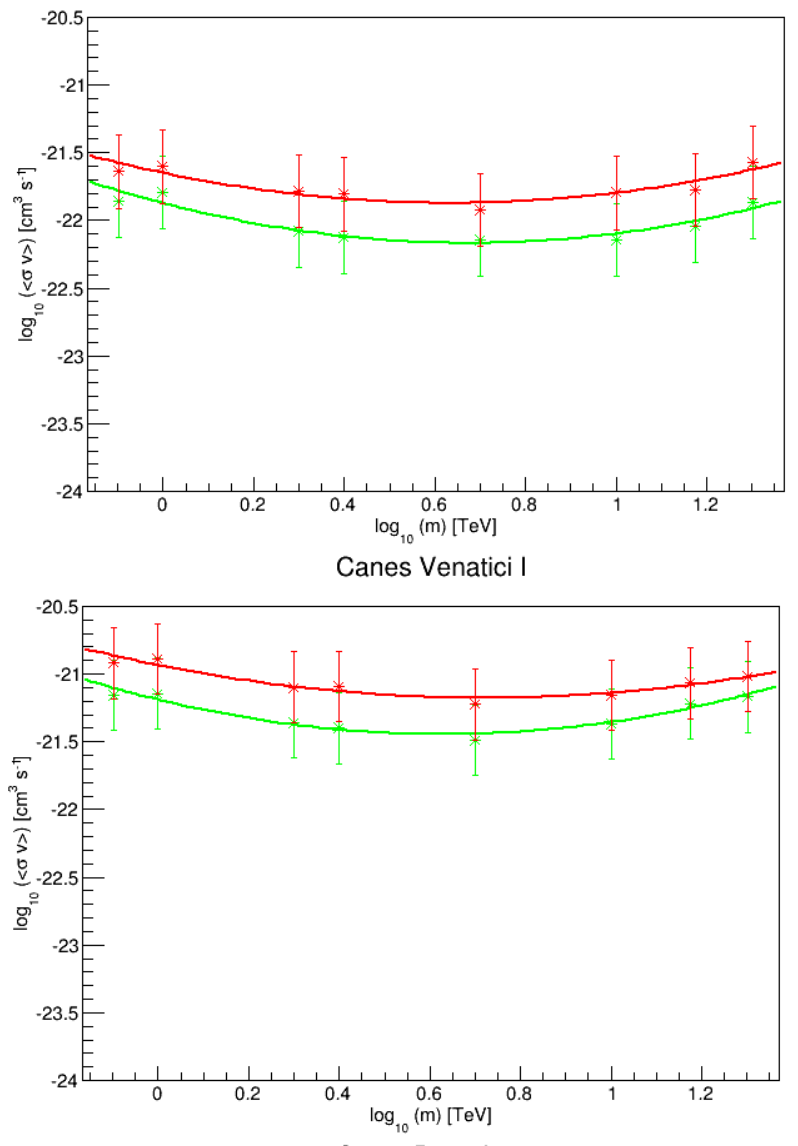

Coma Berenices

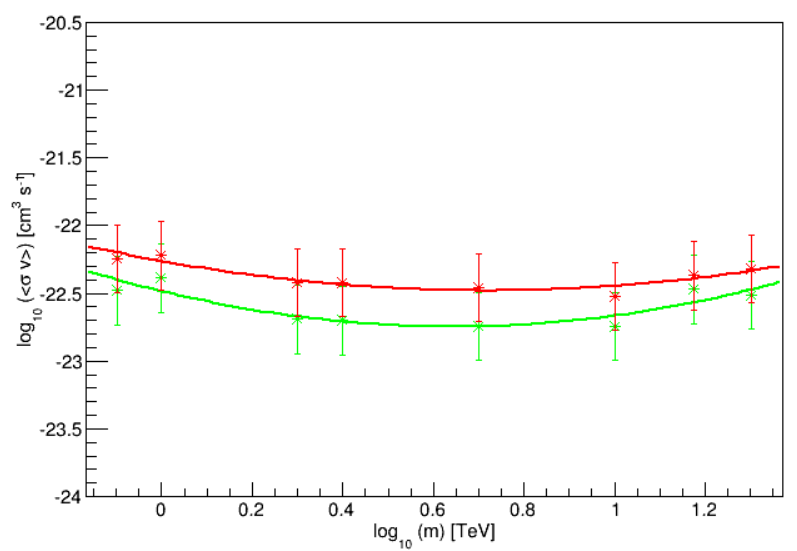

Fonte: Elaborada pela autora. 
Figura 6.11 - Curvas de seção de choque de aniquilação por massa para as 18 fontes estudadas com duas configurações diferentes: significância $3 \sigma$, mínimo de eventos 7 e ruído de fundo $0.03 \%$ e significância $5 \sigma$, mínimo de eventos 10 e ruído de fundo $0.05 \%$. A legenda presente no gráfico da fonte Sculptor se aplica para todos os outros gráficos.

Hercules
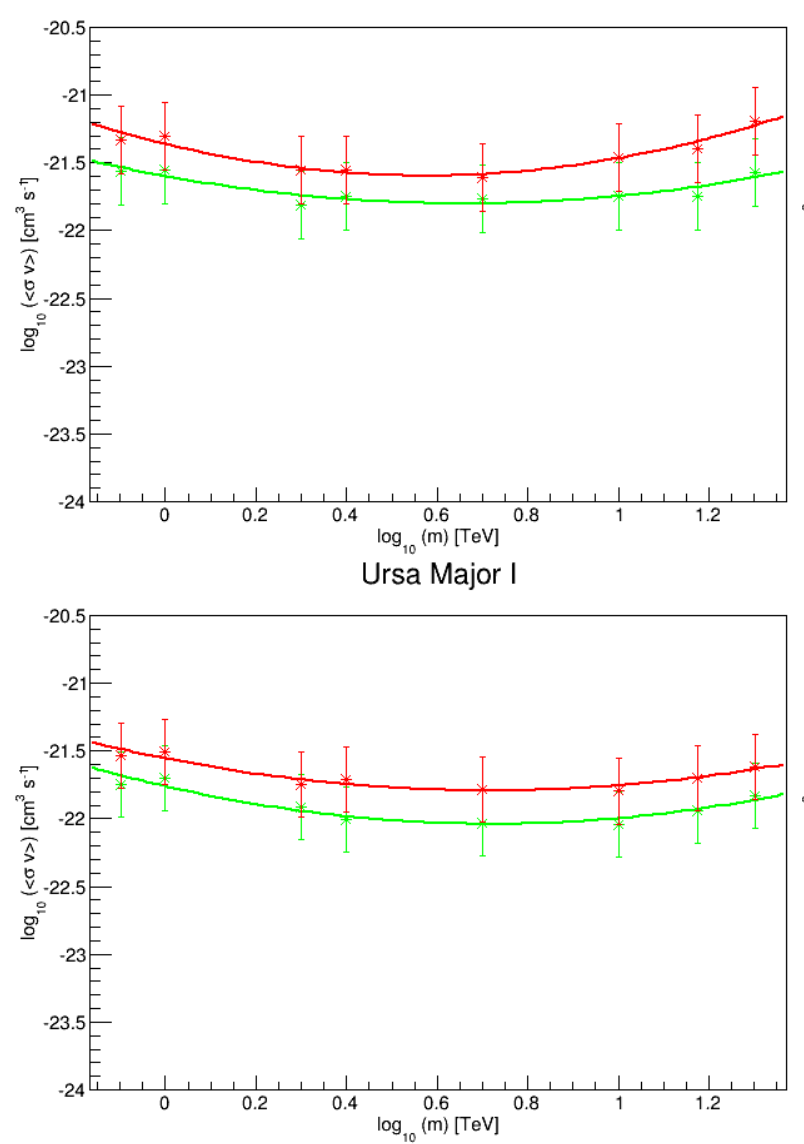

Draco

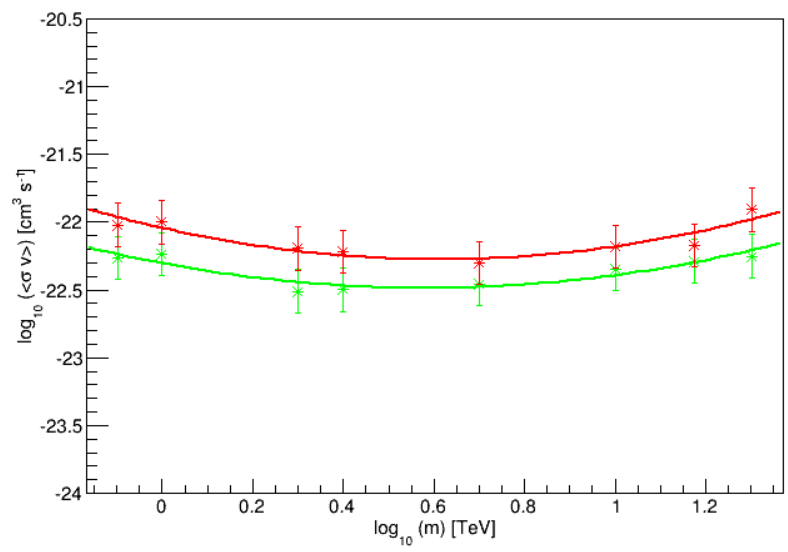

Leo IV
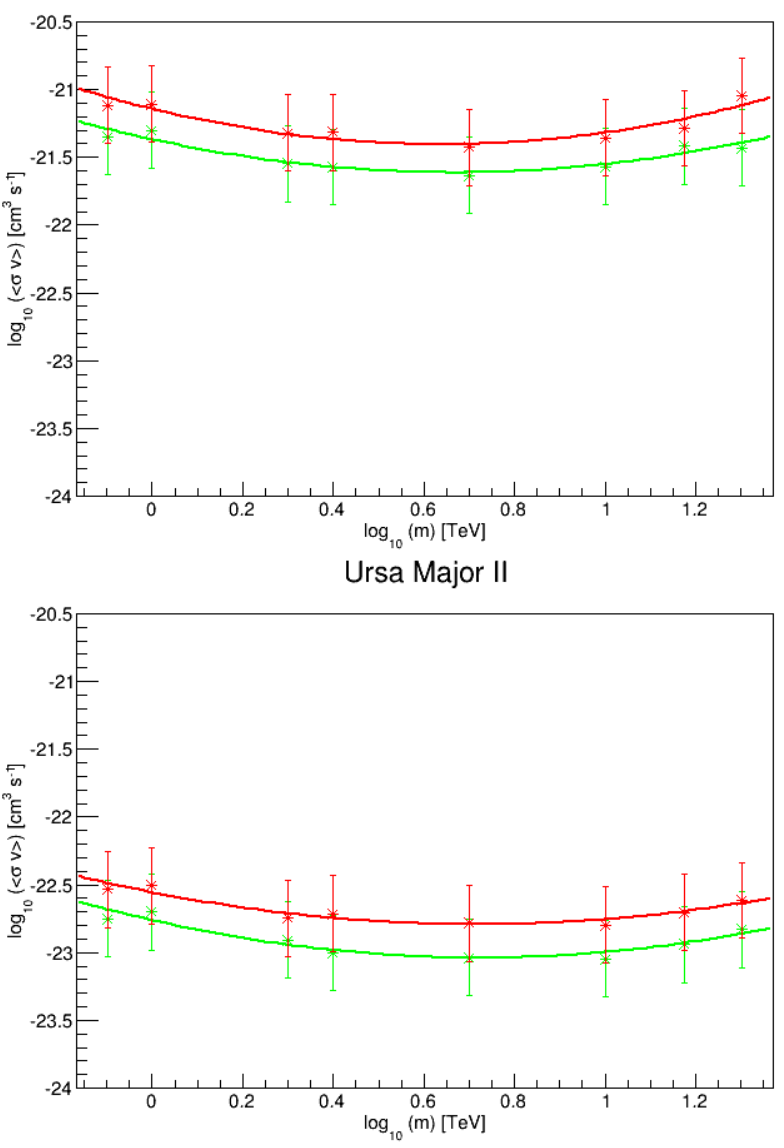

Willman I

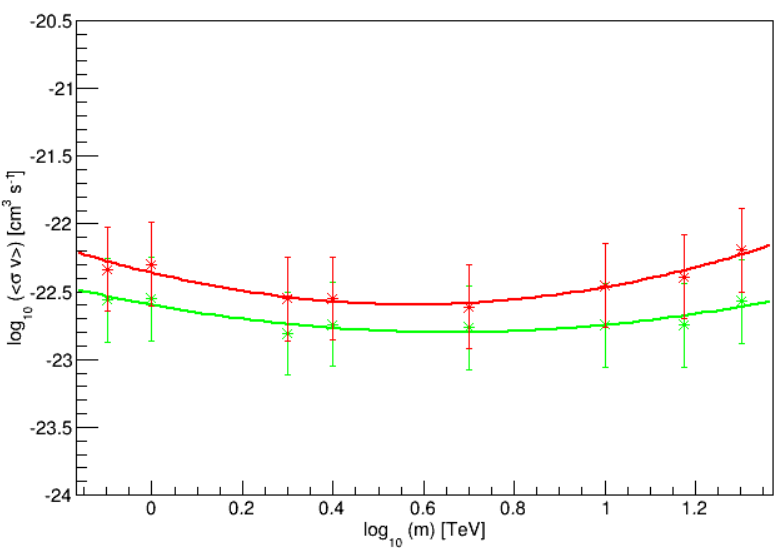

Fonte: Elaborada pela autora. 
Figura 6.12 - Comparação das sensitividades referentes ao CTA (em magenta) e ao FERMI-LAT (verde). A curva referente ao CTA foi calculada com significância $3 \sigma$ e com $50 \mathrm{~h}$ de tempo de observação.

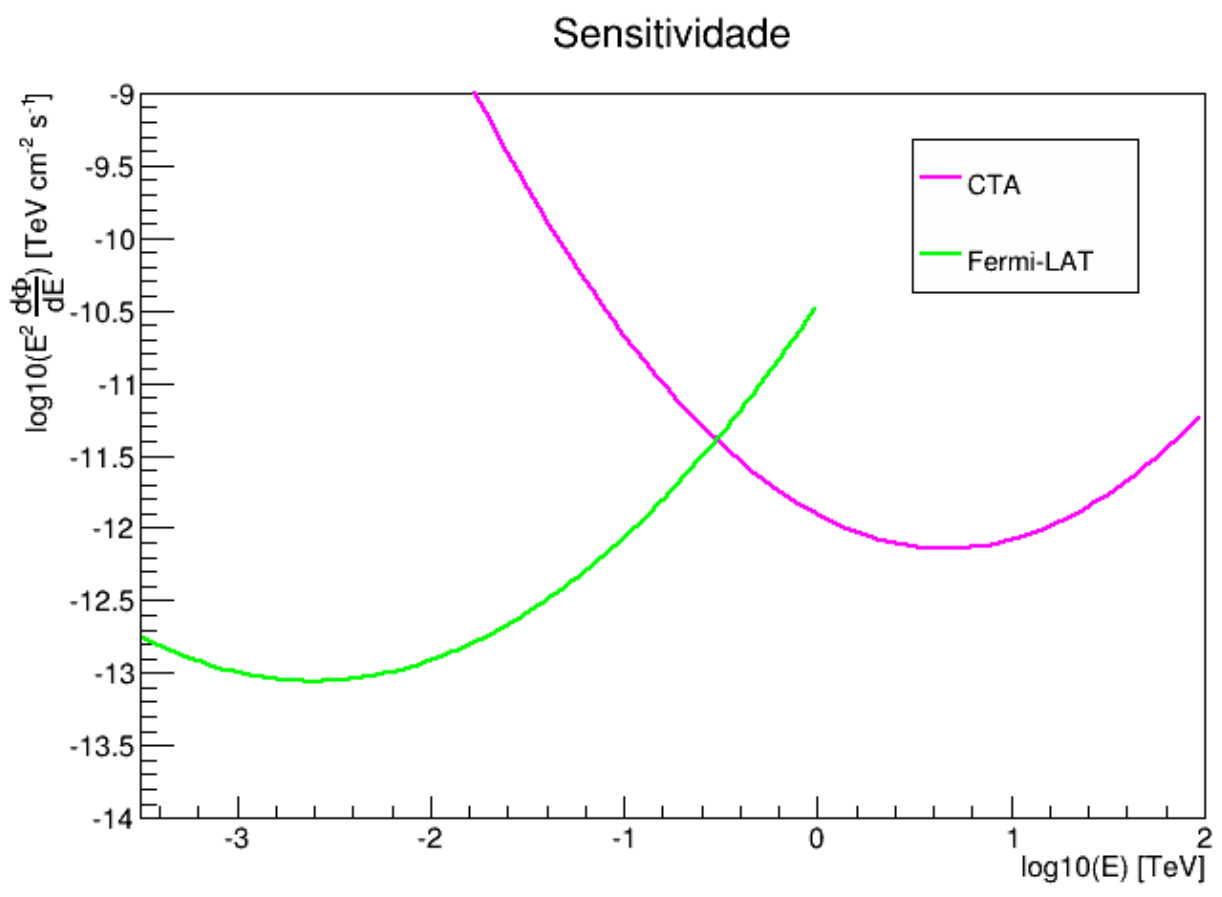

Fonte: Elaborada pela autora.

\subsubsection{Análise da potencialidade do CTA no estudo de fontes me- didas pelo FERMI-LAT}

Analisaremos nessa subseção a possível contribuição do CTA para detecção de matéria escura a partir das fontes estudadas pelo FERMI-LAT em (23).

Vamos considerar primeiramente as sensitividades dos dois telescópios. Para isso, extraímos os dados de limite superior do fluxo diferencial de cada fonte da referência (23). É possível notar no conjunto de dados da referência que há pouca variação no valor do fluxo de fonte para fonte, uma vez que a sensitividade do telescópio não depende da fonte observada. Dessa forma, para fins comparativos, escolhemos a média entre as curvas de fluxo diferencial das 18 galáxias estudadas. Como os dados da referência possuem significância $3 \sigma$, também utilizamos dados de sensitividade do CTA com $3 \sigma$ a partir do programa de simulação apresentados na figura 6.7 Por fim, na figura 6.12 apresentamos o gráfico comparativo de sensitividades do FERMI-LAT e do CTA.

Para os resultados de fontes individuais, seguimos o mesmo procedimento de cálculo das curvas de seção de choque por massa. Para estudarmos a contribuição do CTA, também 
apresentamos os dados da referência nos gráficos a seguir. As figuras 6.13, 6.14 e 6.15 mostram o comportamento das curvas de seção de choque por massa de cada telescópio para cada fonte. Os valores dos pontos obtidos com a simulação do CTA correspondem aos presentes na tabela 6.7 com significância $3 \sigma$. Os valores correspondentes à curva do FERMILAT estão presentes na referência. (23)

Como os fatores astrofísicos $J$ e as parametrizações de $\frac{\mathrm{d} N_{\gamma}}{d E}$ são as mesmas mencionadas até agora, os erros finais nos valores de seção de choque continuam dominados apenas pelo desvio em $J$ pois os desvios propagados a partir da parametrização são compararativamente pequenos.

A contribuição do CTA para o estudo de matéria escura em cada uma dessas fontes é evidente quando consideramos o intervalo em que a sensitividade do CTA é menor do que a do FERMI-LAT. Em todas as fontes, foi possível observar um intervalo de massas correspondente a fluxos inferiores aos do FERMI-LAT possíveis de detecção pelo CTA. Podemos ressaltar fontes com maior potencial para o CTA a partir dos gráficos em 6.13, 6.14 e 6.15. Para classificá-las como ótimas, adotamos o seguinte critério: a contribuição do CTA deve ser limitada no mínimo com um valor de massa $m_{\chi} \approx 2.5 \mathrm{TeV}$. A partir desse valor, pudemos estabelecer as fontes Sculptor, Segue I, Bootes I, Canes Venatici I, Hercules, Ursa Major II e Willman I como fontes com maior potencialidade para a contribuição do CTA. Na tabela 6.8 apresentamos os valores de massa mínima da partícula de matéria escura possível de detecção para cada fonte a partir da delimitação da reta de sensitividade do FERMI-LAT. 
Figura 6.13 - Curvas de seção de choque de aniquilação por massa para as 18 fontes. A reta preta representa o limite superior no valor de seção de choque derivado pelo FERMI-LAT em (23). A curva magenta, os valores de seção de choque calculados com a simulação de resposta do CTA. A legenda presente no gráfico da fonte Sculptor se aplica para todos os outros gráficos.

Sculptor
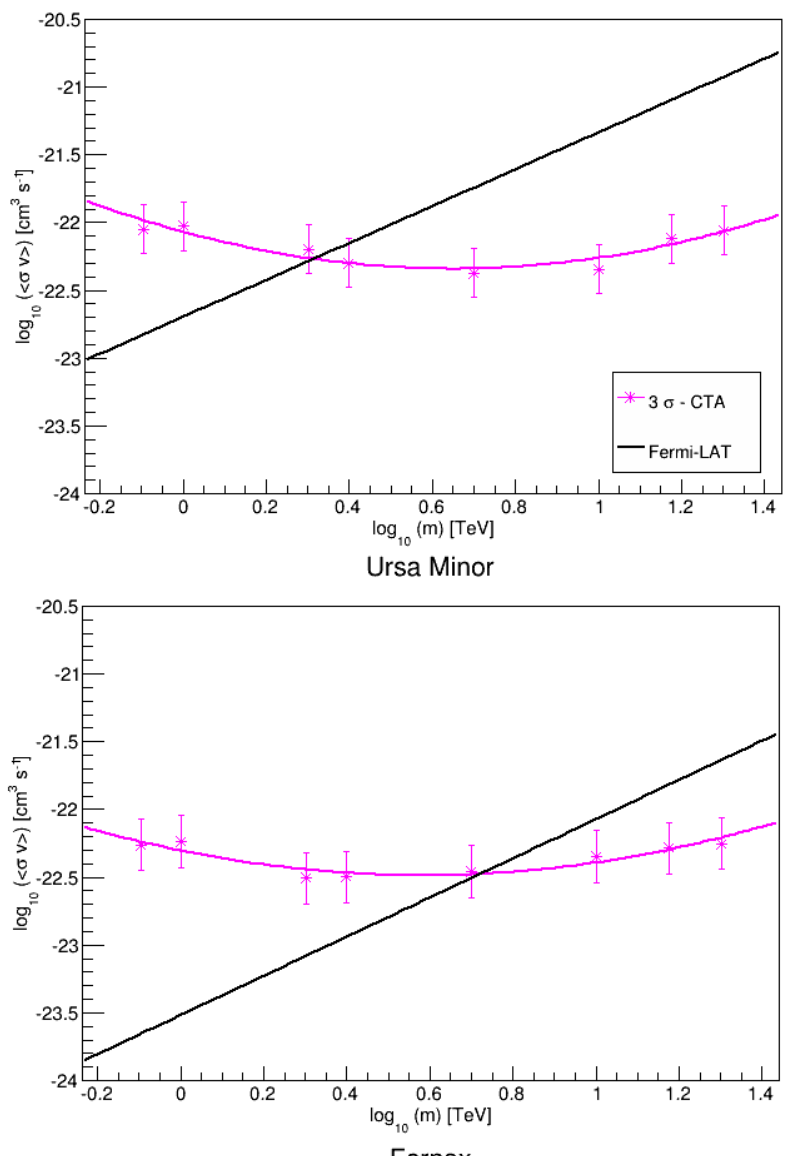

Fornax

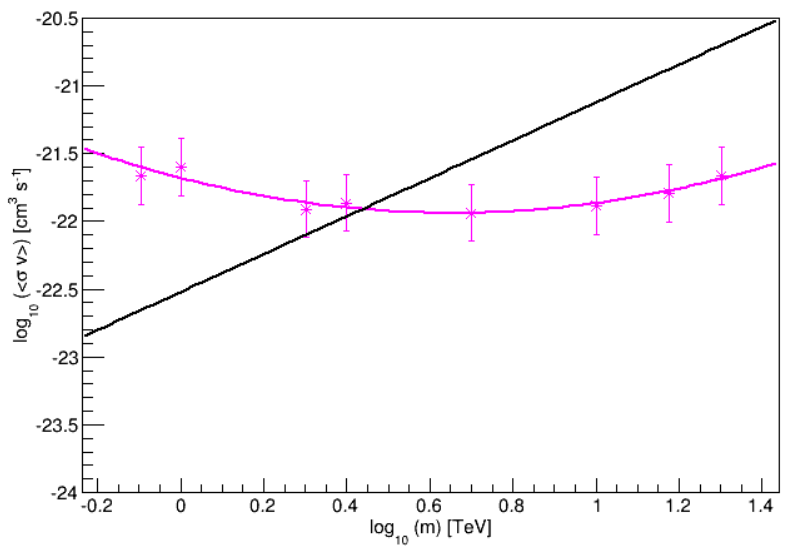

Segue I
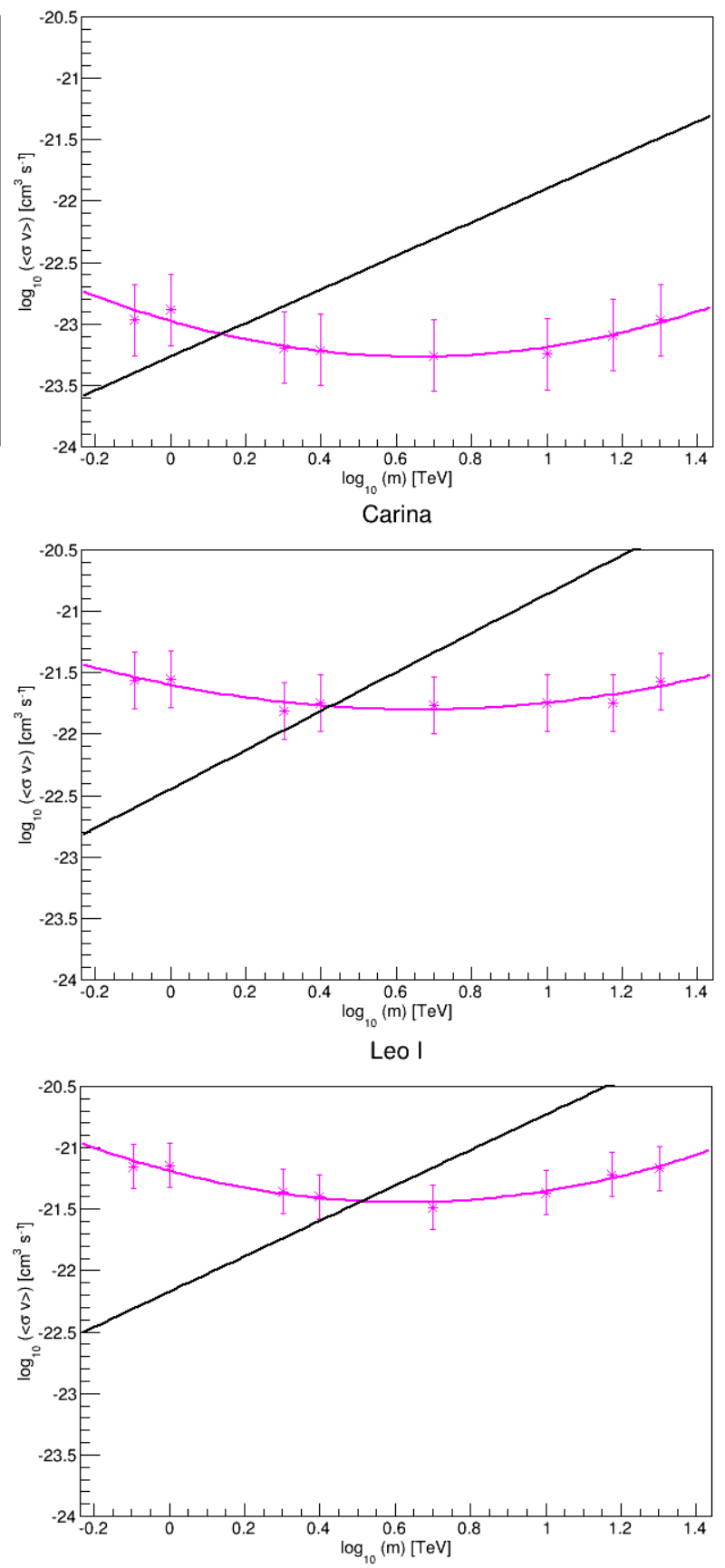

Fonte: Elaborada pela autora. 
Figura 6.14 - Curvas de seção de choque de aniquilação por massa para as 18 fontes. A reta preta representa o limite superior no valor de seção de choque derivado pelo FERMI-LAT em (23). A curva magenta, os valores de seção de choque calculados com a simulação de resposta do CTA. A legenda presente no gráfico da fonte Sculptor se aplica para todos os outros gráficos.

Leo II
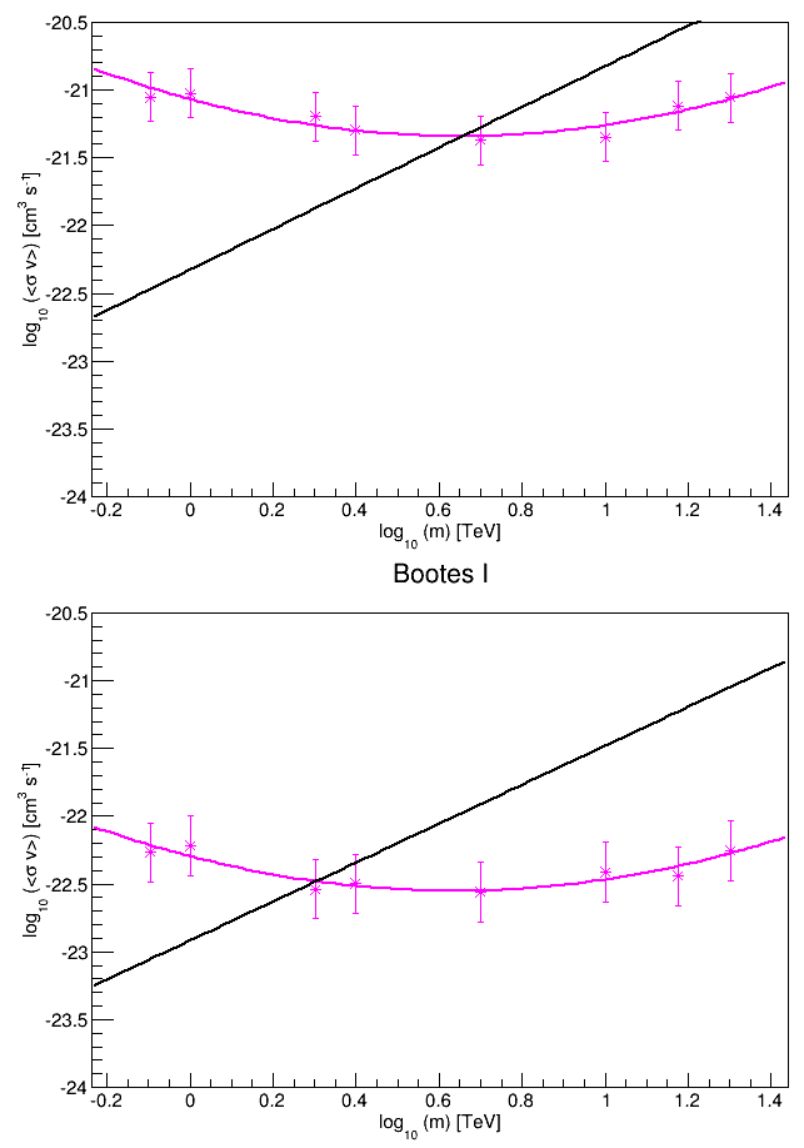

Canes Venatici II

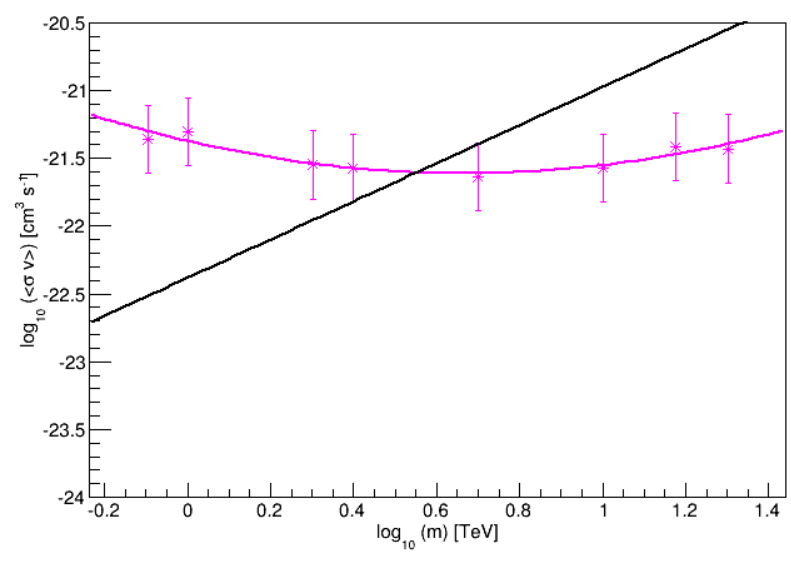

Sextans

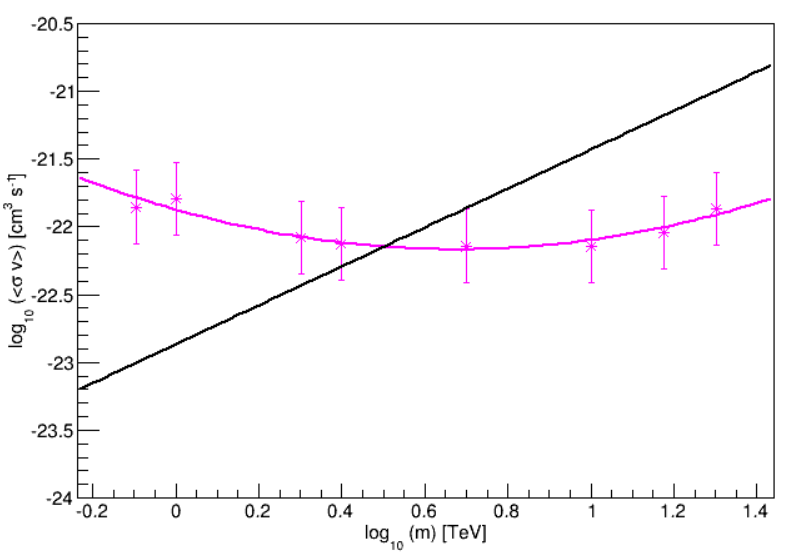

Canes Venatici I

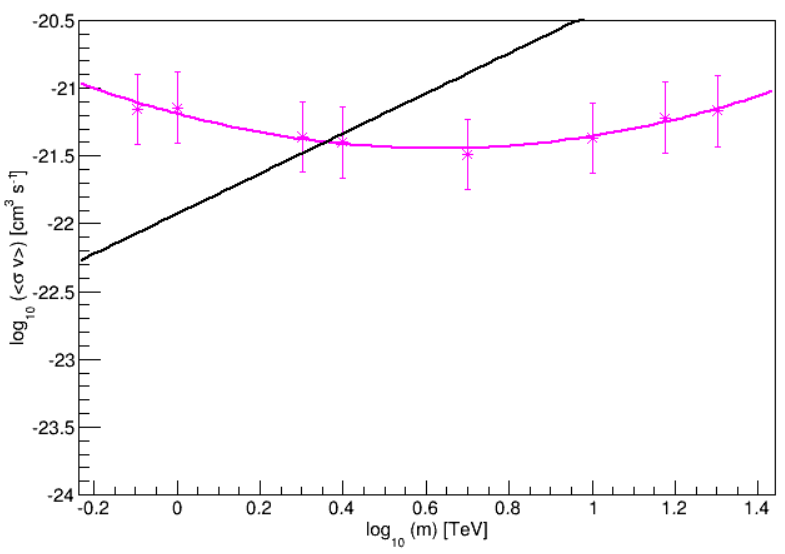

Coma Berenices

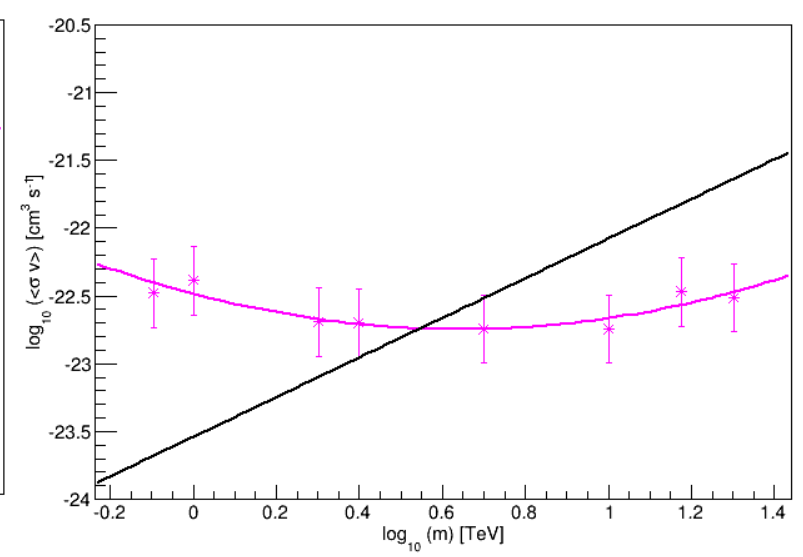

Fonte: Elaborada pela autora. 
Figura 6.15 - Curvas de seção de choque de aniquilação por massa para as 18 fontes. A reta preta representa o limite superior no valor de seção de choque derivado pelo FERMI-LAT em (23). A curva magenta, os valores de seção de choque calculados com a simulação de resposta do CTA. A legenda presente no gráfico da fonte Sculptor se aplica para todos os outros gráficos.

Hercules
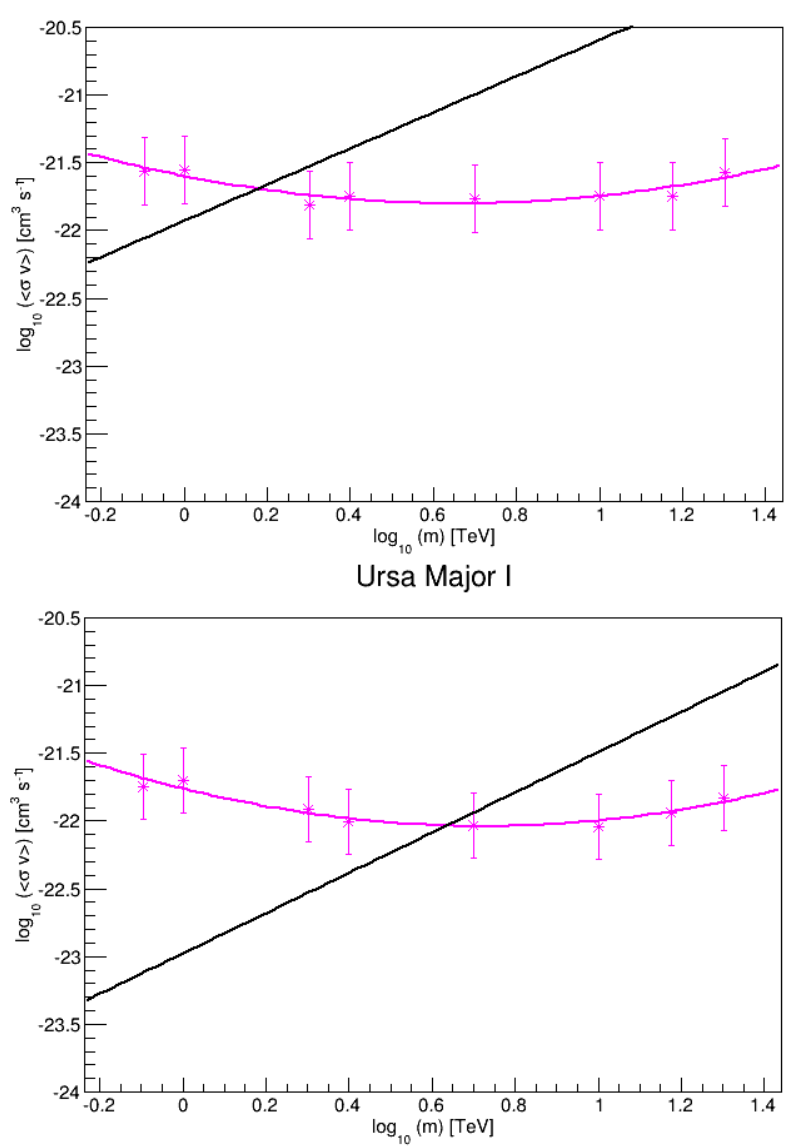

Draco

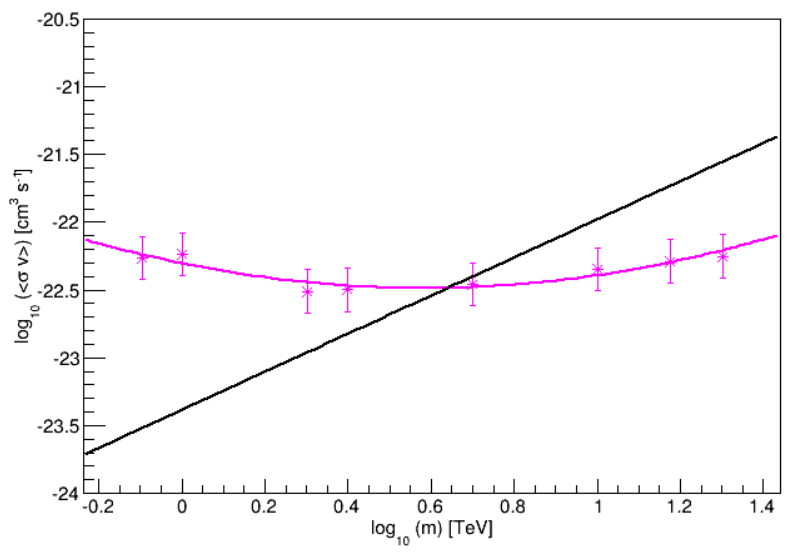

Leo IV
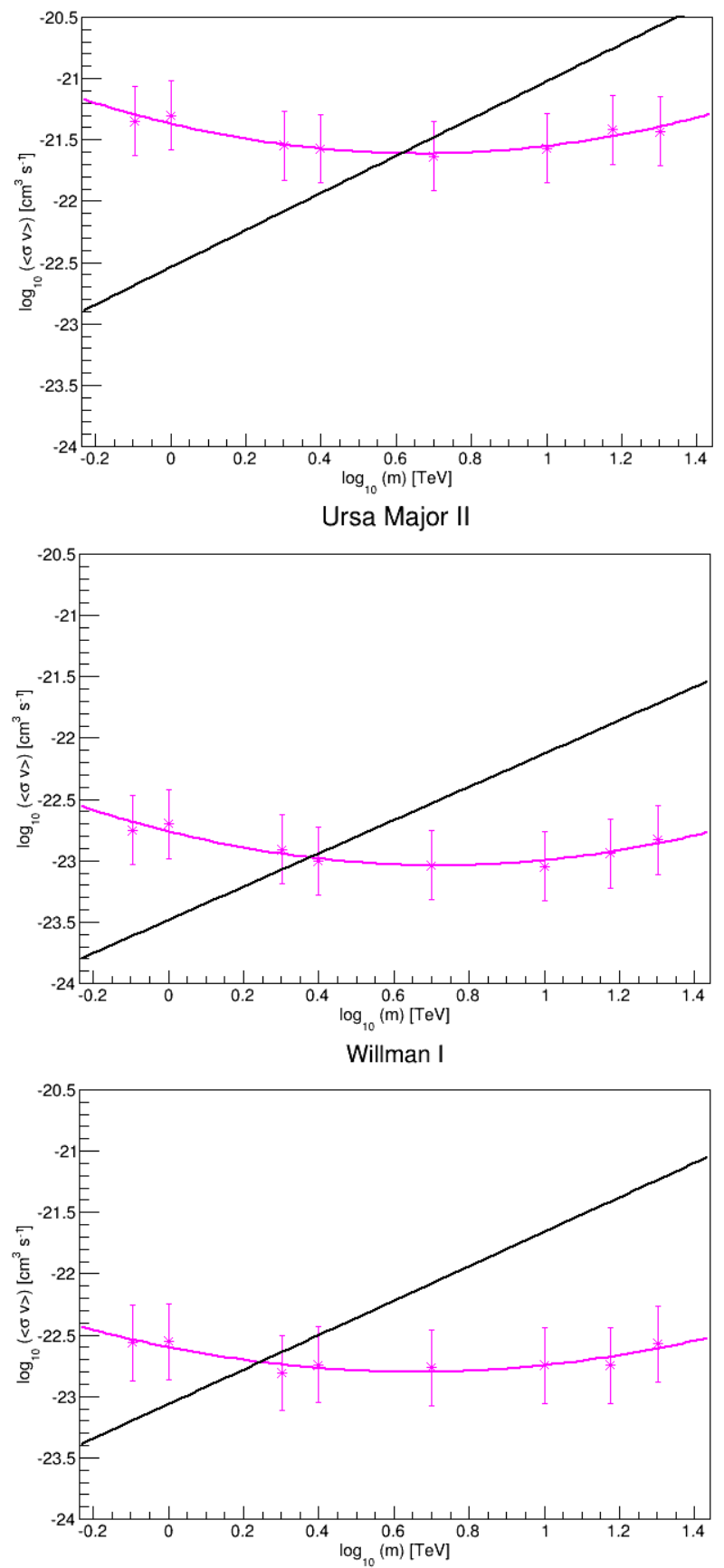

Fonte: Elaborada pela autora. 
Tabela 6.7 - Valores dos pontos obtidos para as galáxias presentes nas figuras 6.9, 6.10 e 6.11 .

\begin{tabular}{ccccc}
\hline \multicolumn{2}{c}{ Sculptor } & \multicolumn{2}{c}{ Segue I } \\
\hline$m_{\chi}(\mathrm{TeV})$ & $<\sigma v>_{3 \sigma}\left(\mathrm{cm}^{3} \mathrm{~s}^{-1}\right)$ & $\left\langle\sigma v>_{5 \sigma}\left(\mathrm{cm}^{3} \mathrm{~s}^{-1}\right)\right.$ & $\left\langle\sigma v>_{3 \sigma}\left(\mathrm{cm}^{3} \mathrm{~s}^{-1}\right)\right.$ & $<\sigma v>_{5 \sigma}\left(\mathrm{cm}^{3} \mathrm{~s}^{-1}\right)$ \\
\hline 0.8 & $8.9_{-3.0}^{+4.6} \cdot 10^{-23}$ & $1.5_{-0.5}^{+0.8} \cdot 10^{-22}$ & $1.1_{+1.0}^{+1.0} \cdot-23$ & $1.8_{-0.9}^{+1.7} \cdot 10^{-23}$ \\
1.0 & $9.4_{-3.2}^{+4.8} \cdot 10^{-23}$ & $1.7_{-0.6}^{+0.9} \cdot 10^{-22}$ & $1.3_{-0.6}^{+1.2} \cdot-23$ & $2.1_{-1.0}^{+2.0} \cdot 10^{-23}$ \\
2.0 & $6.4_{-2.2}^{+3.3} \cdot 10^{-23}$ & $1.0_{-0.3}^{+0.5} \cdot 10^{-22}$ & $6.4_{-3.1}^{-6.1} \cdot-24$ & $1.3_{-0.6}^{+1.2} \cdot 10^{-23}$ \\
2.5 & $5.0_{-1.7}^{+2.6} \cdot 10^{-23}$ & $1.1_{-0.4}^{+0.6} \cdot 10^{-22}$ & $6.1_{-3.0}^{+5.8} \cdot-24$ & $1.3_{-0.6}^{+1.2} \cdot 10^{-23}$ \\
5.0 & $4.2_{-1.4}^{+2.2} \cdot 10^{-23}$ & $7.8_{-2.6}^{+4.0} \cdot 10^{-23}$ & $5.5_{-2.7}^{+5.2} \cdot-24$ & $9.2_{-4.5}^{+8.8} \cdot 10^{-24}$ \\
10.0 & $4.5_{-1.5}^{+2.3} \cdot 10^{-23}$ & $8.5_{-2.9}^{+4.4} \cdot 10^{-23}$ & $5.7_{-2.8}^{+5.4} \cdot-24$ & $1.1_{-0.5}^{+1.0} \cdot 10^{-23}$ \\
15.0 & $7.6_{-2.6}^{+3.9} \cdot 10^{-23}$ & $1.1_{-0.4}^{+0.6} \cdot 10^{-22}$ & $8.1_{-3.9}^{+7.7} \cdot-24$ & $1.6_{-0.8}^{+1.5} \cdot 10^{-23}$ \\
20.0 & $8.8_{-3.0}^{+4.5} \cdot 10^{-23}$ & $1.6_{-0.6}^{+0.8} \cdot 10^{-22}$ & $1.1_{-0.5}^{+1.0} \cdot-23$ & $2.1_{-1.0}^{+1.9} \cdot 10^{-23}$ \\
\hline
\end{tabular}

\section{Ursa Minor}

Carina

\begin{tabular}{ccccc}
\hline$m_{\chi}(\mathrm{TeV})$ & $<\sigma v>_{3 \sigma}\left(\mathrm{cm}^{3} \mathrm{~s}^{-1}\right)$ & $\left\langle\sigma v>_{5 \sigma}\left(\mathrm{cm}^{3} \mathrm{~s}^{-1}\right)\right.$ & $\left\langle\sigma v>_{3 \sigma}\left(\mathrm{cm}^{3} \mathrm{~s}^{-1}\right)\right.$ & $<\sigma v>_{5 \sigma}\left(\mathrm{cm}^{3} \mathrm{~s}^{-1}\right)$ \\
\hline 0.8 & $5.5_{-1.9}^{+3.0} \cdot 10^{-23}$ & $9.5_{-3.4}^{+5.2} \cdot 10^{-23}$ & $2.7_{-1.1}^{+1.9} \cdot 10^{-22}$ & $4.6_{-1.9}^{+3.2} \cdot 10^{-22}$ \\
1.0 & $5.8_{-2.1}^{+3.2} \cdot 10^{-23}$ & $1.0_{-0.4}^{+0.6} \cdot 10^{-22}$ & $2.8_{-1.1}^{+1.9} \cdot 10^{-22}$ & $5.0_{-2.1}^{+3.5} \cdot 10^{-22}$ \\
2.0 & $3.1_{-1.1}^{+1.7} \cdot 10^{-23}$ & $6.4_{-2.3}^{+3.5} \cdot 10^{-23}$ & $1.6_{-0.6}^{+1.1} \cdot 10^{-22}$ & $2.8_{-1.1}^{+1.9} \cdot 10^{-22}$ \\
2.5 & $3.2_{-1.1}^{+1.7} \cdot 10^{-23}$ & $6.1_{-2.1}^{+3.3} \cdot 10^{-23}$ & $1.8_{-0.7}^{+1.3} \cdot 10^{-22}$ & $2.8_{-1.2}^{+1.9} \cdot 10^{-22}$ \\
5.0 & $3.5_{-1.2}^{+1.9} \cdot 10^{-23}$ & $5.0_{-1.8}^{+2.7} \cdot 10^{-23}$ & $1.7_{-0.7}^{+1.2} \cdot 10^{-22}$ & $2.5_{-1.0}^{+1.7} \cdot 10^{-22}$ \\
10.0 & $4.5_{-1.6}^{+2.5} \cdot 10^{-23}$ & $6.6_{-2.3}^{+3.6} \cdot 10^{-23}$ & $1.8_{-0.7}^{+1.3} \cdot 10^{-22}$ & $3.5_{-1.4}^{+2.4} \cdot 10^{-22}$ \\
15.0 & $5.2_{-1.8}^{+2.8} \cdot 10^{-23}$ & $6.7_{-2.4}^{+3.7} \cdot 10^{-23}$ & $1.8_{-0.7}^{+1.3} \cdot 10^{-22}$ & $5.1_{-1.7}^{+2.8} \cdot 10^{-22}$ \\
20.0 & $5.6_{-2.0}^{+3.1} \cdot 10^{-23}$ & $1.2_{-0.4}^{+0.7} \cdot 10^{-22}$ & $2.7_{-1.1}^{+1.9} \cdot 10^{-22}$ & $3.4_{-2.6}^{+4.5} \cdot 10^{-22}$ \\
\hline
\end{tabular}

\section{Fornax}

\begin{tabular}{|c|c|c|c|c|}
\hline$m_{\chi}(\mathrm{TeV})$ & $<\sigma v>_{3 \sigma}\left(\mathrm{cm}^{3} \mathrm{~s}^{-1}\right)$ & $<\sigma v>_{5 \sigma}\left(\mathrm{cm}^{3} \mathrm{~s}^{-1}\right)$ & $<\sigma v>_{3 \sigma}\left(\mathrm{cm}^{3} \mathrm{~s}^{-1}\right)$ & $<\sigma v>_{5 \sigma}\left(\mathrm{cm}^{3} \mathrm{~s}^{-1}\right)$ \\
\hline 0.8 & $2.2_{-0.8}^{+1.3} \cdot 10^{-22}$ & $3.4_{-14}^{+2.3} \cdot 10^{-22}$ & $7.0_{-24}^{+3.6} \cdot 10^{-22}$ & $1.2_{-04}^{+0.6} \cdot 10^{-21}$ \\
\hline 1.0 & $2.5_{-0.9}^{+1.6} \cdot 10^{-22}$ & $4.1_{-1.6}^{+2.6} \cdot 10^{-22}$ & $7.2_{-2.4}^{+3.7} \cdot 10^{-22}$ & $1.3_{-0.4}^{+0.4} \cdot 10^{-21}$ \\
\hline 2.0 & $1.2_{-0.7}^{+7.7} \cdot 10^{-22}$ & $2.6_{-1.0}^{+1.0} \cdot 10^{-22}$ & $4.4_{-1.5}^{+2.3} \cdot 10^{-22}$ & $8.0_{-2}^{+4.1} \cdot 10^{-22}$ \\
\hline 2.5 & $1.4_{-0.9}^{+0.9} \cdot 10^{-22}$ & $2.4_{-09}^{+1.5} \cdot 10^{-22}$ & $4.0_{-13}^{+2.0} \cdot 10^{-22}$ & $8.1_{-28}^{+4.2} \cdot 10^{-22}$ \\
\hline 5.0 & $1.1_{-0.4}^{+0.7} \cdot 10^{-22}$ & $2.0_{-0.7}^{+1.2} \cdot 10^{-22}$ & $3.2_{-1 .}^{+1.7} \cdot 10^{-22}$ & $6.0_{-2}^{+3.1} \cdot 10^{-22}$ \\
\hline 10.0 & $1.3_{-0.5}^{+0.4} \cdot 10^{-22}$ & $2.8_{-1.1}^{+1.8} \cdot 10^{-22}$ & $4.3_{-1.5}^{+2.2} \cdot 10^{-22}$ & $7.0_{-2.4}^{+3.6} \cdot 10^{-22}$ \\
\hline 15.0 & $1.6_{-0.6}^{+1.0} \cdot 10^{-22}$ & $4.5_{-1.7}^{+2.8} \cdot 10^{-22}$ & $6.1_{-2.1}^{+3.1} \cdot 10^{-22}$ & $8.6_{-2.9}^{+4.4} \cdot 10^{-22}$ \\
\hline 20.0 & $2.2_{-0.8}^{+1.4} \cdot 10^{-22}$ & $5.6_{-2.1}^{+3.5} \cdot 10^{-22}$ & $6.8_{-2.3}^{+3.5} \cdot 10^{-22}$ & $9.6_{-3.2}^{+4.9} \cdot 10^{-22}$ \\
\hline
\end{tabular}

Leo II

Canes Venatici I

\begin{tabular}{ccccc}
\hline$m_{\chi}(\mathrm{TeV})$ & $\left\langle\sigma v>_{3 \sigma}\left(\mathrm{cm}^{3} \mathrm{~s}^{-1}\right)\right.$ & $\left\langle\sigma v>_{5 \sigma}\left(\mathrm{cm}^{3} \mathrm{~s}^{-1}\right)\right.$ & $\left\langle\sigma v>_{3 \sigma}\left(\mathrm{cm}^{3} \mathrm{~s}^{-1}\right)\right.$ & $\left\langle\sigma v>_{5 \sigma}\left(\mathrm{cm}^{3} \mathrm{~s}^{-1}\right)\right.$ \\
\hline 0.8 & $8.9_{-3.0}^{+4.6} \cdot 10^{-22}$ & $1.5_{-0.5}^{+0.8} \cdot 10^{-21}$ & $7.0_{-3.1}^{+5.7} \cdot 10^{-22}$ & $1.2_{-0.5}^{+1.0} \cdot 10^{-21}$ \\
1.0 & $9.4_{-3.2}^{+4.8} \cdot 10^{-22}$ & $1.7_{-0.6}^{+0.9} \cdot 10^{-21}$ & $7.2_{-3.2}^{+5.9} \cdot 10^{-22}$ & $1.3_{-0.6}^{+1.1} \cdot 10^{-21}$ \\
2.0 & $6.4_{-2.2}^{+3.3} \cdot 10^{-22}$ & $1.0_{-0.3}^{+0.5} \cdot 10^{-21}$ & $4.8_{-2.0}^{+3.6} \cdot 10^{-22}$ & $8.0_{-3.6}^{+6.6} \cdot 10^{-22}$ \\
2.5 & $5.0_{-1.7}^{+2.6} \cdot 10^{-22}$ & $1.1_{-0.4}^{+0.5} \cdot 10^{-21}$ & $4.0_{-1.8}^{+3.3} \cdot 10^{-22}$ & $8.1_{-3.7}^{+6.7} \cdot 10^{-22}$ \\
5.0 & $4.2_{-1.4}^{+2.2} \cdot 10^{-22}$ & $7.8_{-2.6}^{+4.0} \cdot 10^{-22}$ & $3.2_{-1.5}^{+2.7} \cdot 10^{-22}$ & $6.0_{-2.7}^{+4.9} \cdot 10^{-22}$ \\
10.0 & $4.5_{-1.5}^{+2.3} \cdot 10^{-22}$ & $8.5_{-2.9}^{+4.4} \cdot 10^{-22}$ & $4.3_{-1.9}^{+3.5} \cdot 10^{-22}$ & $7.0_{-3.1}^{+5.7} \cdot 10^{-22}$ \\
15.0 & $7.6_{-2.6}^{+3.9} \cdot 10^{-22}$ & $1.1_{-0.4}^{+0.6} \cdot 10^{-21}$ & $6.1_{-1.7}^{+5.0} \cdot 10^{-22}$ & $8.6_{-3.9}^{+7.0} \cdot 10^{-22}$ \\
20.0 & $8.8_{-3.0}^{+4.5} \cdot 10^{-22}$ & $1.6_{-0.6}^{+0.8} \cdot 10^{-22}$ & $6.8_{-3.1}^{+5.6} \cdot 10^{-22}$ & $9.6_{-4.3}^{+7.9} \cdot 10^{-22}$ \\
\hline
\end{tabular}

\section{Canes Venatici II}

\section{Sextans}

\begin{tabular}{ccccc}
\hline$m_{\chi}(\mathrm{TeV})$ & $<\sigma v>_{3 \sigma}\left(\mathrm{cm}^{3} \mathrm{~s}^{-1}\right)$ & $\left\langle\sigma v>_{5 \sigma}\left(\mathrm{cm}^{3} \mathrm{~s}^{-1}\right)\right.$ & $\left\langle\sigma v>_{3 \sigma}\left(\mathrm{cm}^{3} \mathrm{~s}^{-1}\right)\right.$ & $\left\langle\sigma v>_{5 \sigma}\left(\mathrm{cm}^{3} \mathrm{~s}^{-1}\right)\right.$ \\
\hline 0.8 & $4.4_{-1.9}^{+3.4} \cdot 10^{-22}$ & $7.7_{-3.3}^{+6.0} \cdot 10^{-22}$ & $1.4_{-0.6}^{+1.2} \cdot 10^{-22}$ & $2.3_{-1.1}^{+2.0} \cdot 10^{-22}$ \\
1.0 & $5.0_{-2.2}^{+3.9} \cdot 10^{-22}$ & $7.7_{-3.4}^{+6.0} \cdot 10^{-22}$ & $1.6_{-0.7}^{+1.4} \cdot 10^{-22}$ & $2.5_{-1.2}^{+2.1} \cdot 10^{-22}$ \\
2.0 & $2.8_{-1.2}^{+2.2} \cdot 10^{-22}$ & $4.8_{-2.1}^{+3.7} \cdot 10^{-22}$ & $8.4_{-3.9}^{+7.2} \cdot 10^{-23}$ & $1.6_{-0.8}^{+1.4} \cdot 10^{-22}$ \\
2.5 & $2.7_{-1.2}^{+2.1} \cdot 10^{-22}$ & $4.8_{-2.1}^{+3.7} \cdot 10^{-22}$ & $7.5_{-3.4}^{+6.4} \cdot 10^{-23}$ & $1.6_{-0.7}^{+1.3} \cdot 10^{-22}$ \\
5.0 & $2.3_{-1.0}^{+1.8} \cdot 10^{-22}$ & $3.7_{-1.6}^{+2.9} \cdot 10^{-22}$ & $7.2_{-3.3}^{+6.2} \cdot 10^{-23}$ & $1.2_{-0.6}^{+1.0} \cdot 10^{-22}$ \\
10.0 & $2.7_{-1.2}^{+2.1} \cdot 10^{-22}$ & $4.4_{-1.9}^{+3.4} \cdot 10^{-22}$ & $7.2_{-3.3}^{+6.2} \cdot 10^{-23}$ & $1.6_{-0.7}^{+1.4} \cdot 10^{-22}$ \\
15.0 & $3.8_{-1.7}^{+3.0} \cdot 10^{-22}$ & $5.2_{-2.3}^{+4.0} \cdot 10^{-22}$ & $9.0_{-4.2}^{+7.8} \cdot 10^{-23}$ & $1.7_{-0.8}^{+1.5} \cdot 10^{-22}$ \\
20.0 & $3.7_{-1.6}^{+2.9} \cdot 10^{-22}$ & $9.0_{-3.9}^{+7.0} \cdot 10^{-22}$ & $1.4_{-0.6}^{+1.2} \cdot 10^{-22}$ & $2.7_{-1.2}^{+2.3} \cdot 10^{-22}$ \\
\hline
\end{tabular}


Continuação.

\begin{tabular}{|c|c|c|c|c|}
\hline & \multicolumn{2}{|c|}{ Bootes I } & \multicolumn{2}{|c|}{ Coma Berenices } \\
\hline$m_{\chi}(\mathrm{TeV})$ & $<\sigma v>_{3 \sigma}\left(\mathrm{cm}^{3} \mathrm{~s}^{-1}\right)$ & $<\sigma v>_{5 \sigma}\left(\mathrm{cm}^{3} \mathrm{~s}^{-1}\right)$ & $<\sigma v>_{3 \sigma}\left(\mathrm{cm}^{3} \mathrm{~s}^{-1}\right)$ & $<\sigma v>_{5 \sigma}\left(\mathrm{cm}^{3} \mathrm{~s}^{-1}\right)$ \\
\hline 0.8 & $5.4_{-2.1}^{+3.6} \cdot 10^{-23}$ & $9.6_{-3.8}^{+6.3} \cdot 10^{-23}$ & $3.3_{-1.5}^{+2.6} \cdot 10^{-23}$ & $5.7_{-2.5}^{+4.5} \cdot 10^{-23}$ \\
\hline 1.0 & $6.1_{-2.4}^{+4.0} \cdot 10^{-23}$ & $1.1_{-4.4}^{+7.3} \cdot 10^{-23}$ & $4.1_{-1.8}^{+3.2} \cdot 10^{-23}$ & $6.0_{-2.6}^{+4.5} \cdot 10^{-23}$ \\
\hline 2.0 & $2.9_{-1.1}^{+1.9} \cdot 10^{-23}$ & $6.4_{-2.5}^{+4.2} \cdot 10^{-23}$ & $2.0_{-0.9}^{+1.6} \cdot 10^{-23}$ & $3.8_{-1.7}^{+3.0} \cdot 10^{-23}$ \\
\hline 2.5 & $3.2_{-1.1}^{+2.1} \cdot 10^{-23}$ & $6.0_{-24}^{+4.0} \cdot 10^{-23}$ & $2.0_{-0.9}^{+1.6} \cdot 10^{-23}$ & $3.8_{-17}^{+3.0} \cdot 10^{-23}$ \\
\hline 5.0 & $2.7_{-1.1}^{+1.8} \cdot 10^{-23}$ & $5.0_{-20}^{+3.3} \cdot 10^{-23}$ & $1.8_{-0.8}^{+1.4} \cdot 10^{-23}$ & $3.5_{-1.5}^{+2.7} \cdot 10^{-23}$ \\
\hline 10.0 & $3.9_{-1.5}^{+2.6} \cdot 10^{-23}$ & $5.5_{-2.2}^{+3.6} \cdot 10^{-23}$ & $1.8_{-0.8}^{+1.8} \cdot 10^{-23}$ & $3.0_{-1.3}^{+2.3} \cdot 10^{-23}$ \\
\hline 15.0 & $3.6_{-1.4}^{+2.4} \cdot 10^{-23}$ & $6.7_{-2.7}^{+4.4} \cdot 10^{-23}$ & $3.4_{-1.5}^{+2.6} \cdot 10^{-23}$ & $4.3_{-1.9}^{+3.3} \cdot 10^{-23}$ \\
\hline \multirow[t]{2}{*}{20.0} & $5.6_{-2.2}^{+3.7} \cdot 10^{-23}$ & $7.6_{-3.0}^{+5.0} \cdot 10^{-23}$ & $3.1_{-1.3}^{+2.4} \cdot 10^{-23}$ & $4.8_{-2.1}^{+3.7} \cdot 10^{-23}$ \\
\hline & \multicolumn{2}{|c|}{ Hercules } & \multicolumn{2}{|c|}{ Ursa Major I } \\
\hline$m_{\chi}(\mathrm{TeV})$ & $<\sigma v>_{3 \sigma}\left(\mathrm{cm}^{3} \mathrm{~s}^{-1}\right)$ & $<\sigma v>_{5 \sigma}\left(\mathrm{cm}^{3} \mathrm{~s}^{-1}\right)$ & $<\sigma v>_{3 \sigma}\left(\mathrm{cm}^{3} \mathrm{~s}^{-1}\right)$ & $<\sigma v>_{5 \sigma}\left(\mathrm{cm}^{3} \mathrm{~s}^{-1}\right)$ \\
\hline 0.8 & $2.7_{-1.2}^{+2.1} \cdot 10^{-22}$ & $4.5_{-2.0}^{+3.6} \cdot 10^{-22}$ & $1.8_{-0.8}^{+1.3} \cdot 10^{-22}$ & $2.9_{-1.2}^{+2.1} \cdot 10^{-22}$ \\
\hline 1.0 & $2.8_{-1.2}^{+2.1} \cdot 10^{-22}$ & $5.0_{-2.2}^{+3.9} \cdot 10^{-22}$ & $2.0_{-0.9}^{+1.5} \cdot 10^{-22}$ & $3.1_{-1.3}^{+2.3} \cdot 10^{-22}$ \\
\hline 2.0 & $1.6_{-0.7}^{+1.2} \cdot 10^{-22}$ & $2.8_{-1.2}^{+2.2} \cdot 10^{-22}$ & $1.2_{-0.5}^{+0.9} \cdot 10^{-22}$ & $1.8_{-0.8}^{+1.3} \cdot 10^{-22}$ \\
\hline 2.5 & $1.8_{-0.8}^{+1.4} \cdot 10^{-22}$ & $2.8_{-1.2}^{+2.2} \cdot 10^{-22}$ & $9.9_{-4.2}^{+7.3} \cdot 10^{-23}$ & $1.9_{-0.8}^{+1.4} \cdot 10^{-22}$ \\
\hline 5.0 & $1.7_{-0.8}^{+1.8} \cdot 10^{-22}$ & $2.4_{-1.0}^{+1.2} \cdot 10^{-22}$ & $9.2_{-3.9}^{+6.2} \cdot 10^{-23}$ & $1.7_{-0.7}^{+1.8} \cdot 10^{-22}$ \\
\hline 10.0 & $1.8_{-0.8}^{+1.4} \cdot 10^{-22}$ & $3.6_{-1.5}^{+2.7} \cdot 10^{-22}$ & $9.0_{-3.8}^{+6.6} \cdot 10^{-23}$ & $1.6_{-0.7}^{+1.2} \cdot 10^{-22}$ \\
\hline 15.0 & $1.8_{-0.8}^{+1.4} \cdot 10^{-22}$ & $4.0_{-1.8}^{+3.1} \cdot 10^{-22}$ & $1.1_{-0.5}^{+0.8} \cdot 10^{-23}$ & $2.0_{-0.8}^{+1.5} \cdot 10^{-22}$ \\
\hline \multirow[t]{2}{*}{20.0} & $2.7_{-1.2}^{+2.1} \cdot 10^{-22}$ & $6.4_{-2.8}^{+5.0} \cdot 10^{-22}$ & $1.5_{-0.6}^{+1.1} \cdot 10^{-22}$ & $2.4_{-1.0}^{+1.8} \cdot 10^{-22}$ \\
\hline & \multicolumn{2}{|c|}{ Ursa Major II } & \multicolumn{2}{|c|}{ Leo IV } \\
\hline$m_{\chi}(\mathrm{TeV})$ & $<\sigma v>_{3 \sigma}\left(\mathrm{cm}^{3} \mathrm{~s}^{-1}\right)$ & $<\sigma v>_{5 \sigma}\left(\mathrm{cm}^{3} \mathrm{~s}^{-1}\right)$ & $<\sigma v>_{3 \sigma}\left(\mathrm{cm}^{3} \mathrm{~s}^{-1}\right)$ & $<\sigma v>_{5 \sigma}\left(\mathrm{cm}^{3} \mathrm{~s}^{-1}\right)$ \\
\hline 0.8 & $1.8_{-0.8}^{+1.6} \cdot 10^{-23}$ & $2.9_{-1.4}^{+2.6} \cdot 10^{-23}$ & $4.5_{-2.1}^{+4.1} \cdot 10^{-22}$ & $7.7_{-3.6}^{+6.9} \cdot 10^{-22}$ \\
\hline 1.0 & $2.0_{-0.9}^{+1.8} \cdot 10^{-23}$ & $3.1_{-1.5}^{+2.8} \cdot 10^{-23}$ & $5.0_{-2.4}^{+4.5} \cdot 10^{-22}$ & $7.8_{-3.7}^{+7.0} \cdot 10^{-22}$ \\
\hline 2.0 & $1.2_{-0.6}^{+1.1} \cdot 10^{-23}$ & $1.8_{-0.9}^{+1.6} \cdot 10^{-23}$ & $2.8_{-1.3}^{+2.6} \cdot 10^{-22}$ & $4.8_{-2.3}^{+4.3} \cdot 10^{-22}$ \\
\hline 2.5 & $9.9_{-2.7}^{+9.0} \cdot 10^{-24}$ & $1.9_{-0.9}^{+1.6} \cdot 10^{-23}$ & $2.7_{-1.3}^{+2.4} \cdot 10^{-22}$ & $4.8_{-2.3}^{+4.3} \cdot 10^{-22}$ \\
\hline 5.0 & $9.2_{-4.4}^{+8.4} \cdot 10^{-24}$ & $1.7_{-0.8}^{+1.5} \cdot 10^{-23}$ & $2.3_{-1.1}^{+2.3} \cdot 10^{-22}$ & $3.7_{-1.8}^{+3.4} \cdot 10^{-22}$ \\
\hline 10.0 & $9.0_{-4.3}^{+8.1} \cdot 10^{-24}$ & $1.6_{-0.8}^{+1.4} \cdot 10^{-23}$ & $2.7_{-1.3}^{+2.4} \cdot 10^{-22}$ & $4.4_{-2.1}^{+4.0} \cdot 10^{-22}$ \\
\hline 15.0 & $1.1_{-0.5}^{+1.0} \cdot 10^{-23}$ & $2.0_{-0.9}^{+1.8} \cdot 10^{-23}$ & $3.8_{-1.8}^{+3.5} \cdot 10^{-22}$ & $5.2_{-2.5}^{+4.1} \cdot 10^{-22}$ \\
\hline \multirow[t]{2}{*}{20.0} & $1.5_{-0.7}^{+1.3} \cdot 10^{-23}$ & $2.4_{-1.1}^{+2.2} \cdot 10^{-23}$ & $3.7_{-1.8}^{+3.4} \cdot 10^{-22}$ & $9.0_{-4.3}^{+8.1} \cdot 10^{-22}$ \\
\hline & \multicolumn{2}{|c|}{ Draco } & \multicolumn{2}{|c|}{ Willman I } \\
\hline$m_{\chi}(\mathrm{TeV})$ & $<\sigma v>_{3 \sigma}\left(\mathrm{cm}^{3} \mathrm{~s}^{-1}\right)$ & $<\sigma v>_{5 \sigma}\left(\mathrm{cm}^{3} \mathrm{~s}^{-1}\right)$ & $<\sigma v>_{3 \sigma}\left(\mathrm{cm}^{3} \mathrm{~s}^{-1}\right)$ & $<\sigma v>_{5 \sigma}\left(\mathrm{cm}^{3} \mathrm{~s}^{-1}\right)$ \\
\hline 0.8 & $5.5_{-1.7}^{+2.4} \cdot 10^{-23}$ & $9.5_{-2.9}^{+4.2} \cdot 10^{-23}$ & $2.7_{-1.4}^{+2.8} \cdot 10^{-23}$ & $4.6_{-2.4}^{+4.8} \cdot 10^{-23}$ \\
\hline 1.0 & $5.8_{-1.8}^{+2.6} \cdot 10^{-23}$ & $1.0_{-0.3}^{+0.4} \cdot 10^{-22}$ & $2.8_{-1.4}^{+2.9} \cdot 10^{-23}$ & $5.0_{-2.5}^{+5.2} \cdot 10^{-23}$ \\
\hline 2.0 & $3.1_{-0.9}^{+1.4} \cdot 10^{-23}$ & $6.4_{-2.0}^{+2.8} \cdot 10^{-23}$ & $1.6_{-0.8}^{+1.6} \cdot 10^{-23}$ & $2.8_{-1.4}^{+2.9} \cdot 10^{-23}$ \\
\hline 2.5 & $3.2_{-1.0}^{+1.4} \cdot 10^{-23}$ & $6.0_{-1.9}^{+2.7} \cdot 10^{-23}$ & $1.8_{-0.9}^{+1.9} \cdot 10^{-23}$ & $2.8_{-1.4}^{+2.9} \cdot 10^{-23}$ \\
\hline 5.0 & $3.5_{-1.1}^{+1.6} \cdot 10^{-23}$ & $5.0_{-1.5}^{+2.2} \cdot 10^{-23}$ & $1.7_{-0.9}^{+1.8} \cdot 10^{-23}$ & $2.4_{-1.2}^{+2.5} \cdot 10^{-23}$ \\
\hline 10.0 & $4.5_{-1.4}^{+2.0} \cdot 10^{-23}$ & $6.6_{-2.0}^{+2.9} \cdot 10^{-23}$ & $1.8_{-0.9}^{+1.9} \cdot 10^{-23}$ & $3.5_{-1.8}^{+3.6} \cdot 10^{-23}$ \\
\hline 15.0 & $5.2_{-1.6}^{+2.3} \cdot 10^{-23}$ & $6.7_{-2.1}^{+3.0} \cdot 10^{-23}$ & $1.8_{-0.9}^{+1.9} \cdot 10^{-23}$ & $4.0_{-2.1}^{+4.2} \cdot 10^{-23}$ \\
\hline 20.0 & $5.6_{-1.7}^{+2.5} \cdot 10^{-23}$ & $1.2_{-3.8}^{+5.5} \cdot 10^{-23}$ & $2.7_{-1.4}^{+2.8} \cdot 10^{-23}$ & $6.4_{-3.2}^{+6.6} \cdot 10^{-23}$ \\
\hline
\end{tabular}

Fonte: Elaborado pela autora. 
Tabela 6.8 - Valores de massa mínimos por galáxia possíveis de serem obtidos pelo sinal captado pelo CTA a partir da delimitação do FERMI-LAT em (23).

\begin{tabular}{cc}
\hline fonte & $m_{\chi, \text { mínimo }}(\mathrm{TeV})$ \\
\hline Sculptor & 2.1 \\
Segue 1 & 1.3 \\
Ursa Minor & 5.3 \\
Carina & 2.6 \\
Fornax & 2.7 \\
Leo I & 3.2 \\
Leo II & 6.6 \\
Sextans & 3.1 \\
Bootes I & 2.0 \\
Canes Venatici I & 2.3 \\
Canes Venatici II & 3.6 \\
Coma Berenices & 3.5 \\
Hercules & 1.5 \\
Leo IV & 4.1 \\
Ursa Major I & 4.4 \\
Ursa Major II & 2.4 \\
Draco & 4.4 \\
Willman I & 1.7 \\
\hline
\end{tabular}

Fonte: Elaborado pela autora. 


\section{Conclusões}

Apresentamos nessa dissertação de mestrado um estudo sobre a possibilidade de detecção indireta de matéria escura com telescópios Cherenkov, avaliando o peso de algumas características estatísticas para o resultado sobre propriedades intrínsecas da possível partícula.

Discutimos primeiramente as bases teóricas que constituem o cenário de matéria escura priorizando um modelo específico de estudo: a detecção indireta de WIMPs, especificamente via a aniquilação de neutralinos. Focando nas expectativas futuras com relação a esta área em particular, descrevemos alguns aspectos importantes sobre o Observatório CTA, tendo como base a alteração de sua sensitividade com relação a aspectos físicos e configurações experimentais, como a escolha do arranjo dos telescópios, o tempo de observação considerado para a medida, entre outros.

\subsection{Cálculo de limites superiores na seção de choque de aniquilação}

Para validar a proposta de detecção indireta de neutralinos, estudamos limites superiores da seção de choque de aniquilação obtidos com os telescópios MAGIC e VERITAS, apresentando um método para o cálculo de seção de choque com os dados estabelecidos nas referências.

Utilizamos os dados referentes aos fatores astrofísicos $J$, aos fluxos integrais de raios gama de cada fonte e aos modelos adotados nas referências para o perfil de produção de raios gama por aniquilação de partículas de matéria escura. Elaboramos um código para aproximar uma função correspondente à taxa de produção de raios gama e integrá-la nos limites de energia propostos em cada telescópio, determinando assim os valores de limite de seção de choque de aniquilação.

A reprodução dos resultados a partir dos dados do MAGIC proporcionou um primeiro contato com o cálculo do limite de seção de choque de aniquilação. Os erros associados aos valores foram obtidos por propagação de erro do desvio do fator astrofísico $J$ e do desvio 
cometido na aproximação da função do perfil de produção de raios gama. Porém, as diferenças obtidas entre o valor calculado e o valor apresentado na referência (28) para o limite de seção de choque de aniquilação para o canal $b \bar{b}$ não foram aceitáveis. Verificamos todos os passos envolvidos no cálculo com a autora da referência que julgou os resultados obtidos como satisfatórios.

Os resultados apresentados a partir dos dados do VERITAS foram valores próximos aos estabelecidos na referência (21). Porém, os erros associados só puderam ser determinados através dos erros finais dos limites superiores da seção de choque da referência. Tais erros consideram o desvio cometido no modelo de distribuição de matéria escura através do fator astrofísico $J$. Com os resultados obtidos, pudemos validar os passos envolvidos no cálculo do limite da seção de choque.

Foi possível estabelecer uma base de entendimento dos cálculos envolvendo os limites superiores da seção de choque de aniquilação cumprindo a proposta dessa etapa da dissertação.

\subsection{Previsão do limite superior da seção de choque de aniquilação medido pelo CTA}

Posteriormente, partimos para a possibilidade de detecção pelo Observatório CTA a partir do programa de simulação de resposta elaborado por membros da colaboração. Começamos o entendimento do programa e análises focando no tempo de observação da medida realizada. Com base em resultados de seção de choque de aniquilação para $T=100 \mathrm{~h}$ para 3 fontes distintas, derivamos para as mesmas fontes a curva de seção de choque de aniquilação por massa para $T=50 \mathrm{~h}$ elaborando algoritmos e implementações para realização dos cálculos mais detalhados envolvidos no valor final de seção de choque de aniquilação.

Uma vez com os algoritmos e processos bem definidos, analisamos 18 fontes e suas propriedades estudadas pelo telescópio FERMI-LAT em duas etapas distintas. Primeiramente, realizamos um estudo sobre o efeito de características estatísticas como a significância, número mínimo de eventos por dado coletado e porcentagem de ruído de fundo que compõe o sinal captado. Foi possível notar uma diferença clara entre as curvas com significâncias de $3 \sigma$ e de $5 \sigma$ para o valor final de seção de choque de aniquilação. As curvas referentes à significância $5 \sigma$ apresentam valores de seção de choque mais restritos porque estão envolvidas com a confiança no resultado. 
Por fim, analisamos a contribuição futura do CTA para as fontes mencionadas, estudando o comportamento entre as curvas de sensitividade do Fermi-LAT e do CTA, destacando entre as 18 fontes analisadas aquelas com maior potencial para detecção indireta de matéria escura. A potencialidade das fontes estudadas pelo CTA é verificada no intervalo de valores de massa abaixo do delimitado pelo FERMI-LAT, correspondendo a melhor sensitividade proporcionada pelo CTA. Isso significa que em todas as fontes citadas é possível obter uma contribuição do CTA para os futuros resultados de seção de choque de aniquilação e massa da partícula de matéria escura, porém algumas fontes apresentam maior potencialidade, como por exemplo Sculptor, Segue I, Bootes I, Canes Venatici I, Hercules, Ursa Major II e Willman I.

\subsection{Conclusões Gerais}

Nesta dissertação de mestrado foi possível estudar um modelo específico de matéria escura que proporciona a caracterização da possível partícula através de sua seção de choque de aniquilação. Estudamos a potencialidade do futuro Observatório CTA para a detecção de matéria escura enfatizando sua contribuição para a área em questão. Mostramos o efeito de configurações de cunho estatístico para o resultado final dos valores de seção de choque de aniquilação. Através das simulações de respostas e das fontes previamente estudadas pelo FERMI-LAT, evidenciamos quais destas tem maior potencialidade de detecção de matéria escura. 


\section{REFERÊNCIAS}

1 ÖPIK, E. An estimate of the distance of the Andromeda Nebula. Astrophysical Journal, v. 55, p. 406,1922 . doi:10.1086/142680.

2 OORT, J. H. The force exerted by the stellar system in the direction perpendicular to the galactic plane and some related problems. Bulletin of the Astronomical Institutes of the Netherlands, v. 6, p. 249, 1932.

3 ZWICKY, F. Die Rotverschiebung von extragalaktischen Nebeln. Helvetica Physica Acta, p.110-127, 1933. doi:10.5169/seals-110267.

4 RUBIN, V. C.; FORD, W. KENT, J. Rotation of the Andromeda Nebula from a spectroscopic survey of emission regions. Astrophysical Journal, v. 159, p. 379, 1970. doi:10.1086/150317.

5 BERTONE, G. Particle dark matter: observations, models and searches. New York: Academic Press, 2010.

6 MAGIC Collaboration, The MAGIC Telescopes, Disponível em:. <https://magic.mpp. mpg.de/>. Acesso em: 19 jan. 2015.

7 H.E.S.S. Collaboration, High Energy Stereoscopic System, Disponível em:. <https:// wWw.mpi-hd.mpg.de/hfm/HESS/>. Acesso em: 19 jan. 2015.

8 VERITAS Collaboration, Very Energetic Radiation Imaging Telescope Array System, Disponível em:. <http://veritas.sao.arizona.edu/>. Acesso em: 19 jan. 2015.

9 CARROLL, B. W.; OSTLIE, D. A. An introduction to modern astrophysics. 2nd. ed. California: Pearson Addison-Wesley, 2007.

10 BEGEMAN, K.; A.H. BROEILS; SANDERS, R. Extended rotation curves of spiral galaxies: dark haloes and modified dynamics. Monthly Notices of the Royal Astronomical Society, v. 249, p. 523-537, 1991. doi: 10.1093/mnras/249.3.523.

11 BERTIN, G. Dynamic of Galaxies. 2nd. ed. New York: Cambrigde University Press, 2000.

12 NASA CHANDRA X-Ray Observatory, NASA's flagship mission for $X-r$, Disponível em:. $<$ http://chandra.harvard.edu/>. Acesso em: 19 jan. 2015. 
13 CLOWE, D.; BRADAC, M.; GONZALEZ, A. H.; MARKEVITCH, M.; RANDALL, S. W.; JONES, C.; ZARITSKY, D. A direct empirical proof of the existence of dark matter. Astrophysical Journal, v. 648, p. 109-113, 2006. doi:10.1086/508162.

14 BRADAC, M.; CLOWE, D.; GONZALEZ, A. H.; MARSHALL, P.; FORMAN, W.; JONES, C.; MARKEVITCH, M.; RANDALL, S.; SCHRABBACK, T.; ZARITSKY, D. Strong and weak lensing united III: measuring the mass distribution of the merging galaxy cluster 1E0657-56. Astrophysical Journal, v. 652, p. 937-947, 2006. doi:10.1086/508601.

15 BAUMANN, D. The physics of inflation. 2015. Disponivel em: <http://www.damtp.cam.ac.uk/user/db275/TEACHING/INFLATION/Lectures.pdf> Acesso em: 12 maio 2015.

16 PENZIAS, A. A.; WILSON, R. W. A measurement of excess antenna temperature at 4080 Mc/s. Astrophysical Journal, v. 142, p. 419, 1965. doi:10.1086/148307.

17 PLANCK Collaboration et al. Overview of products and scientific results. Astronomy Astrophysics, v. 571, p. 1-48, 2013. doi: 10.1051/0004-6361/201321529.

18 DODELSON, S. Modern cosmology. New York: Academic Press, 2003. ISBN: 978-0-12219141-1.

19 AN, J.; ZHAO, H. Fitting functions for dark matter density profiles. Monthly Notices of the Royal Astronomical Society, v. 428, p. 2805-2811, 2012. doi:10.1093/mnras/sts175.

20 NAVARRO, J. F.; FRENK, C. S.; WHITE, S. D. M. The structure of cold dark matter halos. Astrophysical Journal, v. 462, p. 563, 1996. doi:10.1086/177173.

21 WOOD, M. D. An indirect search for dark matter with VERITAS, 2010. p. 222. Ph.D. Thesis (Astronomy) - University of California, California, 2010.

22 DORO, M. et al. Dark matter and fundamental physics with the Cherenkov Telescope Array. Astroparticle Physics, v. 43, p. 189-214, 2013. doi:10.1016/j.astropartphys.2012.08.002.

23 ACKERMANN, M. et al. Dark matter constraints from observations of 25 Milky Way satellite galaxies with the Fermi Large Area Telescope. Physical Review D, v. 89, p. 1-46, 2014. doi:10.1103/PhysRevD.89.042001.

24 CHARBONNIER, A.; COMBET, C.; DANIEL, M.; FUNK, S.; HINTON, J. A.; MAURIN, D.; POWER, C.; READ, J. I.; SARKAR, S.; WALKER, M. G.; WILKINSON, M. I. Dark matter profiles and annihilation in dwarf spheroidal galaxies: prospectives for present and future gamma-ray observatories - I. the classical dwarf spheroidal galaxies. Monthly Notices of the Royal Astronomical Society, v. 418, n. 3, p. 1526-1556, 2011. doi:10.1111/j.13652966.2011.19387.x. 
25 AHARONIAN, $F$. et al. HESS observations of the galactic center region and their possible dark matter interpretation. Physical Review Letters, v. 97, p. 1-5, 2006. doi:10.1103/PhysRevLett.97.221102.

26 HERNQUIST, L. An analytical model for spherical galaxies and bulges. Astrophysical Journal, v. 356, p. 359, 1990. doi:10.1086/168845.

27 NAVARRO, J. F.; LUDLOW, A.; SPRINGEL, V.; WANG, J.; VOGELSBERGER, M.; WHITE, S. D. M.; JENKINS, A.; FRENK, C. S.; HELMI, A. The diversity and similarity of simulated cold dark matter haloes. Monthly Notices of the Royal Astronomical Society, v. 402, p. 21-34, 2010. doi:10.1111/j.1365-2966.2009.15878.x.

28 ALEKSlć, J. Optimized dark matter searches in deep observations of segue 1 with MAGIC, 2013, p.197, Ph.D. Thesis - Universitat Autònoma de Barcelona, Barcelona, 2013.

29 CIRELLI, M.; PANCI, P.; SERPICO, P. D. Diffuse gamma ray constraints on annihilating or decaying Dark Matter after Fermi. Nuclear Physics B, v. 840, n. 1-2, p.284-303, 2010. doi:10.1016/j.nuclphysb.2010.07.010.

30 VIVIER, M. Indirect searches for dark matter annihilations toward dwarf spheroidal galaxies with VERITAS. 2011. Disponível em: <http://arxiv.org/pdf/1110.6615v2.pdf> Acesso em: 12 maio 2015.

31 BERGSTRöM, L.; BERTONE, G.; CONRAD, J.; FARNIER, C.; WENIGER, C. Investigating gamma-ray lines from dark datter with future observatories. Journal of Cosmology and Astroparticle Physics, v. 2012, p. 12, 2012. doi:10.1088/1475-7516/2012/11/025.

32 BAER, H.; TATA, X. Weak scale supersymmetry. Cambridge, UK: Cambridge University Press, 2012. doi:10.2277/0521857864.

33 ANTUNES, A. C. B. The cold dark matter model with cosmological constant and the flatness constraint. Brazilian Journal of Physics, v. 40, n. 4, p.3-7, 2010.

34 THOMAS, S. A.; ABDALLA, F. B.; LAHAV, O. Upper bound of $0.28 \mathrm{ev}$ on neutrino masses from the largest photometric redshift survey. Physical Review Letters, v. 105, p. 031301, July 2010. doi:10.1103/PhysRevLett.105.031301.

35 ABAZAJIAN, K. N. Resonantly produced 7 kev sterile neutrino dark matter models and the properties of milky way satellites. Physical Review Letters, v. 112, p. 161303, Apr 2014. doi:10.1103/PhysRevLett.112.161303.

36 PECCEI, R. D.; QUINN, H. R. CP conservation in the presence of pseudoparticles. Physical Review Letters, v. 38, p. 1440-1443, 1977. doi:10.1103/PhysRevLett.38.1440. 
37 SáNCHEZ-CONDE, M. A.; PANEQUE, D.; BLOOM, E.; PRADA, F.; DOMÍNGUEZ, A. Hints of the existence of axionlike particles from the gamma-ray spectra of cosmological sources. Physical Review D, v. 79, p. 1-16, 2009. doi:10.1103/PhysRevD.79.123511.

38 ACERO, F. et al. Localizing the VHE $\gamma$-ray source at the galactic centre. Monthly Notices of the Royal Astronomical Society, v. 402, p. 1877-1882, 2010. doi:10.1111/j.13652966.2009.16014.x.

39 FERMI Collaboration, FERMI - Gamma-ray Space Telescope, Disponível em: < <ttps: //www-glast.stanford.edu/P. Acesso em: 19 jan. 2015.

40 MAX PLANCK Society, Max Planck Gesellschaft, Disponível em:. <http://www.mpg. de/en/>. Acesso em: 21 jan. 2015.

41 LHC Collaboration, Large Hadron Collider, Disponível em: < http://home.web.cern. ch/about>. Acesso em: 20 jan. 2015.

42 XENON Collaboration, The XENON dark matter project, Disponível em:. <http:// xenon.astro.columbia.edu/>. Acesso em: 20 jan. 2015.

43 DAMA Collaboration, The DAMA project, Disponível em: < <http://people.roma2. infn.it/ dama/web/det.html>. Acesso em: 20 jan. 2015.

44 CDMS Collaboration, Cryogenic Dark Matter Search, Disponível em:. <http://cdms. berkeley.edu/>. Acesso em: 20 jan. 2015.

45 ACKERMANN, M. et al. Fermi LAT search for dark matter in gamma-ray lines and the inclusive photon spectrum. Physical Review D, v. 86, 2012. doi:10.1103/PhysRevD.86.022002.

46 ALEKSlć, J.; ALVAREZ, E. Searches for dark matter annihilation signatures in the Segue 1 satellite galaxy with the MAGIC-I telescope. Journal of Cosmology and Astroparticle Physics, v. 2011, n. 06, p. 035-035, 2011. doi:10.1088/1475-7516/2011/06/035.

47 ABAZAJIAN, K. N.; KAPLINGHAT, M. Detection of a gamma-ray source in the Galactic Center consistent with extended emission from dark matter annihilation and concentrated astrophysical emission. Physical Review D, v. 86, 2012. doi:10.1103/PhysRevD.86.083511.

48 ACKERMANN, M. et al. Constraining dark matter models from a combined analysis of Milky Way satellites with the Fermi Large Area Telescope. Physical Review Letters, v. 107, p. 1-7, 2011. doi:10.1103/PhysRevLett.107.241302.

49 ZAHARIJAS, J. et al., Constraints on the galactic halo dark matter from Fermi-LAT diffuse measurements, Astrophysical Journal, v.761, p.91, 2012. doi:10.1088/0004-637X/761/2/91. 
50 WOOD, M. et al. A search for dark matter annihilation with the Whipple $10 \mathrm{~m}$ Telescope. Astrophysical Journal, v. 678, n. 2, p. 594-605, 2008. doi:10.1086/529421.

51 VERITAS Collaboration. Search for VHE gamma-ray emission from dwarf spheroidal galaxies. Astrophysical Journal, v. 720, p. 1174-1180, 2010. doi:10.1088/0004-637X/720/2/1174.

52 AHARONIAN, $F$ et al. A search for a dark matter annihilation signal towards the Canis Major overdensity with H.E.S.S., Astrophysical Journal, v. 691, p. 175-181, 2009. doi:10.1088/0004-637X/691/1/175.

53 PULLEN, A. R.; CHARY, R. R.; KAMIONKOWSKI, M. Search with EGRET for a gamma ray line from the Galactic center. Physical Review D, v. 76, p. 1-8, 2007. doi:10.1103/PhysRevD.76.063006.

$54 \mathrm{ABDO}, \mathrm{A}$. A. et al. Fermi large area telescope search for photon lines from 30 to $200 \mathrm{GeV}$ and dark matter implications. Physical Review Letters, v. 104, p. 17-22, 2010. doi:10.1103/PhysRevLett.104.091302.

55 VERTONGEN, G.; WENIGER, C. Hunting dark matter gamma-ray lines with the fermi lat. Journal of Cosmology and Astroparticle Physics, v. 2011, 2011. doi: 10.1088/1475$7516 / 2011 / 05 / 027$.

56 BRINGMANN, T.; WENIGER, C. Gamma ray signals from dark matter: concepts, status and prospects. Physics of the Dark Universe, v. 1, p. 194-217, 2012. doi:10.1016/j.dark.2012.10.005.

57 SPIE Members, SPIE - International Society for optics and photonics, Disponível em:. $<$ http://spie.org/x10.xml?WT.svl=tn7>. Acesso em: 11 maio 2015.

58 CTA Collaboration, Cherenkov Telescope Array, Disponível em: <https://portal. cta-observatory.org/Pages/Home.aspx >. Acesso em: 13 maio 2015.

59 ACTIS, M. et al. Design concepts for the Cherenkov Telescope Array CTA: an advanced facility for ground-based high-energy gamma-ray astronomy. Experimental Astronomy, v. 32, p. 193-316, 2011. doi:10.1007/s10686-011-9247-0.

60 ACHARYA, B. S. et al. Introducing the CTA concept. Astroparticle Physics, v. 43, p. 3-18, 2013. doi:10.1016/j.astropartphys.2013.01.007.

61 BERNLÖHR, K. et al. Monte Carlo design studies for the Cherenkov Telescope Array. Astroparticle Physics, v. 43, p. 171-188, 2013. doi:10.1016/j.astropartphys.2012.10.002.

62 KARLSRUHE INSTITUE OF TECHNOLOGY, CORSIKA - Cosmic Ray Simulations for Kascade, Disponível em: <https://web.ikp.kit.edu/corsika/>. Acesso em: 15 mar. 2015. 
63 GONDOLO, P.; EDSJO, J.; ULLIO, P.; BERGSTROM, L.; SCHELKE, M.; BALTZ, E. A. DarkSUSY: computing supersymmetric dark matter properties numerically. Journal of Cosmology and Astroparticle Physics, p. 35, 2004. doi:10.1088/1475-7516/2004/07/008.

64 BORMISOFT, Digitizelt, Disponível em: <http://www.digitizeit.de/>. Acesso em: 29 jan. 2015.

65 EUROPEAN ORGANIZATION FOR NUCLEAR RESEARCH - CERN, ROOT - Data Analysis Framework, Disponível em: <https://root.cern.ch/drupal/>.

66 CEMBRANOS, J A R, A.; DOBADO, A.; LINEROS, R. A.; MAROTO, A. L. Photon spectra from WIMP annihilation. Physical Review D, v. 83, 2011. doi:10.1103/PhysRevD.83.083507.

67 WOLFRAM RESEARCH, Wolfram Mathematica, Disponível em: <http://www. wolfram.com/mathematica/ >. Acesso em: 22 mar. 2015.

68 HELOU, G.; MADORE, B. F., NED - NASA/IPAC EXTRAGALACTIC DATABASE, Disponível em:. <http://ned.ipac.caltech.edu/>. Acesso em: 23 junho 2015. 